\title{
QUICKEST DETECTION PROBLEMS FOR BESSEL PROCESSES ${ }^{1}$
}

\author{
By Peter Johnson AND Goran PESKIR
}

\section{The University of Manchester}

Consider the motion of a Brownian particle that initially takes place in a two-dimensional plane and then after some random/unobservable time continues in the three-dimensional space. Given that only the distance of the particle to the origin is being observed, the problem is to detect the time at which the particle departs from the plane as accurately as possible. We solve this problem in the most uncertain scenario when the random/unobservable time is (i) exponentially distributed and (ii) independent from the initial motion of the particle in the plane. The solution is expressed in terms of a stopping time that minimises the probability of a false early detection and the expected delay of a missed late detection.

\section{CONTENTS}

1. Introduction . . . . . . . . . . . . . . . . . . . . . . . . . . . . . . . . . . . . . 1003

2. Outline of the paper . . . . . . . . . . . . . . . . . . . . . . 1006

3. Formulation of the problem . . . . . . . . . . . . . . . . . . . . . 1008

4. Measure change . . . . . . . . . . . . . . . . . . . . . . . . . . . . . . . . . 1012

5. Mayer formulation . . . . . . . . . . . . . . . . . . . . . . . . . . . . 1016

6. Reduction to canonical PDE . . . . . . . . . . . . . . . . . . . . . . . . . . . . . . 1019

7. Time change . . . . . . . . . . . . . . . . . . . . . . . . . . . 1025

8. Monotonicity of the optimal stopping boundary . . . . . . . . . . . . . . . . . 1028

9. Continuity of the value function . . . . . . . . . . . . . . . . . . . . . 1034

10. Smooth fit . . . . . . . . . . . . . . . . . . . . . . . . . . . 1036

11. Regularity of the value function . . . . . . . . . . . . . . . . . . . . . . . 1039

12. Continuity of the optimal stopping boundary _ . . . . . . . . . . . . . . . 1043

13. Free-boundary problem . . . . . . . . . . . . . . . . . . . . . . . . 1046

14. Nonlinear integral equation . . . . . . . . . . . . . . . . . . . . . . . . . . 1048

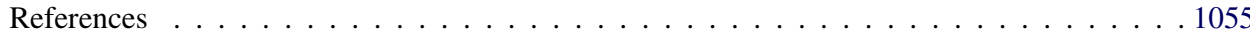

1. Introduction. Imagine the motion of a Brownian particle that initially takes place in a two-dimensional plane and then after some random/unobservable

Received September 2015; revised March 2016.

${ }^{1}$ Supported by a grant from the British Engineering and Physical Sciences Research Council (EPSRC).

MSC2010 subject classifications. Primary 60G40, 60J60, 60H30; secondary 35K67, 45G10, $62 \mathrm{C} 10$.

Key words and phrases. Quickest detection, Brownian motion, Bessel process, optimal stopping, parabolic partial differential equation, free-boundary problem, smooth fit, entrance boundary, nonlinear Fredholm integral equation, the change-of-variable formula with local time on curves/surfaces. 
time $\theta$ continues in the three-dimensional space (Figure 1). Assuming that only the distance of the particle to the origin is being observed (Figure 2), the problem is to detect the time $\theta$ at which the particle departs from the plane as accurately as possible (neither too early nor too late). The purpose of the present paper is to derive the solution to this problem when $\theta$ is assumed to be (i) exponentially distributed and (ii) independent from the initial motion of the particle in the plane.

Denoting the distance of the Brownian particle to the origin by $X$, it is well known that $X$ may be viewed as a Bessel process of dimension 2 which then changes to dimension 3 at time $\theta$. We study the problem above by embedding it into the more general setting where a Bessel process $X$ of dimension $\delta_{0} \geq 2$ changes its dimension to $\delta_{1}>\delta_{0}$ at time $\theta$. In these cases, 0 is known to be an entrance boundary point for $X$ viewed as a diffusion process in $[0, \infty)$ where $X$ is also known to be recurrent for $\delta_{0}=2$ and transient for $\delta_{1}>2$. Our methods are developed to treat these cases and we will leave all other theoretical possibilities of $\delta_{0} \neq \delta_{1} \in[0, \infty)$ open for future development.

The error to be minimised over all stopping times $\tau$ of $X$ is expressed as the linear combination of the probability of the false alarm $\mathrm{P}_{\pi}(\tau<\theta)$ and the expected detection delay $\mathrm{E}_{\pi}(\tau-\theta)^{+}$where $\pi \in[0,1]$ denotes the probability that $\theta$ has already occurred at time 0 . This problem formulation of quickest detection dates back to [18] and has been extensively studied to date (see [19] and the references therein). The linear combination represents the Lagrangian and once the optimal stopping problem has been solved in this form it will also lead to the solution of the constrained problems where an upper bound is imposed on either the probability of the false alarm or the expected detection delay, respectively. A canonical example is the standard Brownian motion with one constant drift changing to another. This problem has also been solved in finite horizon (see [8] and the references therein). Books [20], Section 4.4, and [15], Section 22, contain expositions of these results and provide further details and references. In all these problems, however, the signal-to-noise ratio (defined as the difference between the new drift and the old drift divided by the diffusion coefficient) is constant. This is no longer the case in the quickest detection problem of the present paper and to our knowledge this is the first time that such a problem has been solved in the literature.

A more general problem formulation for one-dimensional diffusion processes when one nonconstant drift changes to another has been considered in the recent paper [9]. This reference serves as a starting point for the present paper and for future reference we will also make it explicit in the analysis below which arguments/results are applicable/valid in the general case as well. To recognise the Markovian structure in the optimal stopping problem stated above, one considers the posterior probability distribution process $\Pi$ of $\theta$ given $X$, as well as the posterior probability distribution ratio process $\Phi$ of $\theta$ given $X$, in addition to the observed process $X$. The two processes $\Pi$ and $\Phi$ are in one-to-one correspondence (the latter stretching the state space $[0,1]$ to $[0, \infty]$ of the former) so that one of them is Markov if and only if the other is Markov. This is the case when the 


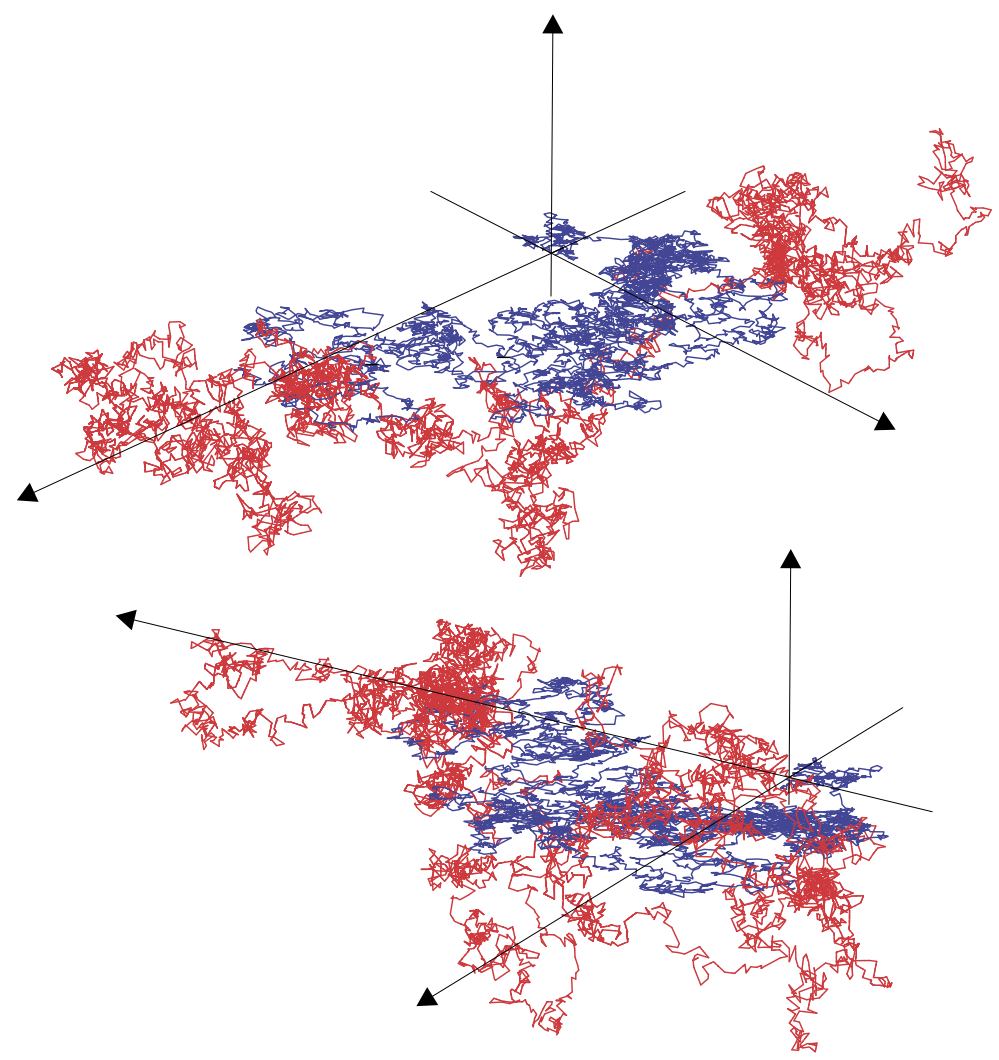

FIG. 1. Simulated motion of a Brownian particle (viewed from two angles) that initially takes place in a two-dimensional plane (blue line) and then after some random/unobservable time $\theta$ continues in the three-dimensional space (red line).

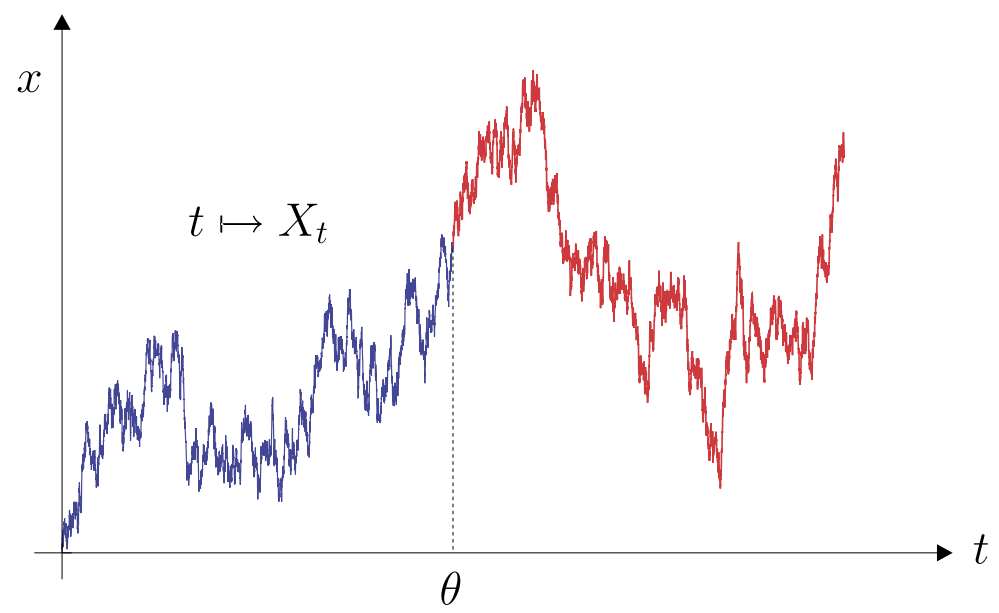

FIG. 2. Distance to the origin of the Brownian particle from Figure 1 above. 
signal-to-noise ratio is constant. On the other hand, if the signal-to-noise ratio is not constant, then typically both $\Pi$ and $\Phi$ fail to be Markov processes. To remedy the situation, as noted in [9], one needs to account for $X$ and then both $(\Pi, X)$ and $(\Phi, X)$ become Markov processes. This shows that if the signal-to-noise ratio is not constant, as in the quickest detection problem of the present paper, then the optimal stopping problem under consideration is inherently/fully two-dimensional, and hence more difficult. Finding and fully characterising the solution to this problem is the main/principal result of the present paper.

2. Outline of the paper. The exposition of the material is organised as follows. In Section 3, we formulate the optimal stopping problem and recall the stochastic differential equations for $\Pi, \Phi, L$ and $X$ from [9], Section 2, where $L$ is the likelihood ratio process which provides a link from $\Pi$ and $\Phi$ to the observed process $X$. The stochastic differential equations for $\Pi, \Phi$ and $X$ are expressed in terms of the innovation process (standard Brownian motion) so that the stochastic differential equations for both $(\Pi, X)$ and $(\Phi, X)$ are fully coupled. This makes the analysis of the optimal stopping problem more complicated.

In Section 4, we show that a measure change from $\mathrm{P}_{\pi}$ to $\mathrm{P}^{\infty}$ (corresponding to $\theta=\infty$ formally) simplifies the matters in that the stochastic differential equations for both $(\Pi, X)$ and $(\Phi, X)$ get uncoupled in the second component. This is an important step that abandons the innovation process and makes the subsequent analysis possible. A similar approach to the Poisson disorder problem has been undertaken in [2] where the quickest detection problem was formulated starting with $\mathrm{P}^{\infty}$ and then built on further from there. The difference in the present case is that we find/determine the Radon-Nikodym derivative which reformulates the traditional construction of the problem in terms of $\mathrm{P}^{\infty}$ although clearly the two approaches ought to be equivalent.

The resulting optimal stopping problem is expressed in Lagrange form for $(\Phi, X)$ and in Section 5 we disclose its Mayer formulation (see [15], Section 6, for the terminology). Since the stochastic differential equations for $\Phi$ and $X$ are driven by the same Brownian motion we know that the resulting infinitesimal generator equation must be of parabolic type and in Section 6 we describe this reduction in both probabilistic and analytic terms. This enables us to rewrite the optimal stopping problem (of Sections 4 and 5) in terms of a new Markov process $(U, \Phi)$ where the process $U$ is of bounded variation. Some precision is needed in this context as strictly speaking the partial differential equation is parabolic only off the curve where the integrand forming $U$ takes value 0 (to change its sign). A similar reduction to the canonical form has been carried out in [9] in a more general setting so that the resulting partial differential equation contains mixed derivatives as well. Nonexistence of the mixed derivatives simplifies some proofs in the sequel (Section 12).

In Section 7, we recall that a Bessel process of dimension $\delta \geq 2$ can be time changed into a geometric Brownian motion. This is a well-known result dating 
back to [21] and the time change itself plays a crucial role to derive monotonicity of the optimal stopping boundary in Section 8. It may be instructive to compare this derivation with the derivation given in the recent paper [1] where similar time change arguments were used in a somewhat different setting (leaving a nonmartingale case treated here as an open problem). The proof of monotonicity verifies by different/rigorous means the implication stated in [9], Proposition 4.1, that the optimal stopping boundary is decreasing if the signal-to-noise ratio is decreasing. In Section 8, we also derive further properties of the optimal stopping boundary (its finiteness, its limiting behaviour at zero and infinity, and its regularity for the stopping set). To establish its monotonicity and regularity, we make use of the time change arguments combined with a comparison theorem for multidimensional stochastic differential equations given in [7].

Using the regularity of the optimal stopping boundary we show in Section 9 that the value function of the optimal stopping problem is continuous. Similarly, in Section 10 we show that smooth fit holds in both directions of $(\Phi, X)$ at the optimal stopping boundary. Since the process $(\Phi, X)$ is in one-to-one correspondence with the process $(U, \Phi)$ it follows that all the results proved for the process $(\Phi, X)$ and its optimal stopping problem extend in a one-to-one way to the results for the process $(U, \Phi)$ and its optimal stopping problem (implying also that the optimal stopping problems are equivalent). The advantage of the process $(U, \Phi)$ lies in the fact that its first component is either increasing or decreasing when $(U, \Phi)$ is off a specified curve so that the resulting motion of $(U, \Phi)$ is "circular around" the curve. This reflects the fact that the underlying partial differential equation of its infinitesimal generator is not globally parabolic (but only locally off the curve).

In Section 11, we present direct probabilistic arguments (based on the regularity of the optimal stopping boundary alone) which show that the value function is globally continuously differentiable (see [3] for extensions and broader analysis). Exploiting further the local parabolicity of the equation through the connection between $(\Phi, X)$ and $(U, \Phi)$ this enables us to infer that the value function for the latter process is twice continuously differentiable with respect the second argument up to the optimal stopping boundary (within the continuation set). The combined results of Sections 10 and 11 are then used in Section 12 to derive continuity of the optimal stopping boundary itself (see [14] for extensions of these arguments to elliptic equations). Making use of the existence and uniqueness results for partial differential equations of parabolic type (cf. [11]) this is then used in Section 13 to complete the analytic setting and formulate the free-boundary problem which stands in one-to-one correspondence with the optimal stopping problem.

In Section 14, we exploit the regularity properties of the value function derived in Section 11 combined with the local parabolicity of the equation [through the connection between $(\Phi, X)$ and $(U, \Phi)]$ and monotonicity of the optimal stopping boundary derived in Section 8 . These facts justify the use of the local timespace formula from [13] which then shows that the optimal stopping boundary 
solves a nonlinear Fredholm integral equation in the same way as the optimal stopping boundary in the time-space problems of optimal stopping solves a nonlinear Volterra integral equation (see [15], Chapters VI-VIII, and the references therein). The reason for the appearance of the Fredholm equations instead of the Volterra equations is that the partial differential equation of the infinitesimal generator is not globally parabolic (this will also be the case when the equation is elliptic). This means that the equation is not globally evolutionary, and hence the integration needs to be performed over the whole state space. The replacement of the nonlinear Volterra integral equation by the nonlinear Fredholm integral equation is canonical and the phenomenon holds in general for more complicated (elliptic) and higher dimensional problems as well.

In Section 14, we further verify that the proof of uniqueness for the nonlinear Volterra integral equation given originally in [12] and developed further for example in [4], Section 3.10, extends to the nonlinear Fredholm integral equation. This shows that the optimal stopping boundary can be characterised as the unique solution to the nonlinear Fredholm integral equation and there is a closed tripleintegral representation for the value function expressed in terms of the optimal stopping boundary. These results also establish uniqueness of the solution to the free-boundary problem formulated in Section 13 in addition to its existence.

Concluding this section, we can say that the present study addresses a number of key issues when dealing with fully two-dimensional problems of optimal stopping for diffusion processes and their connections with (locally) parabolic partial differential equations. The methodology for tackling these problems is of general probabilistic interest and the arguments developed in the proofs should be applicable in similar two-dimensional settings.

3. Formulation of the problem. In this section, we formulate the quickest detection problem under consideration and recall stochastic differential equations for the underlying stochastic processes (cf. [9], Section 2). These considerations will be completed in the next section by a change of measure argument.

1. We consider a Bayesian formulation of the problem where it is assumed that one observes a sample path of the Bessel process $X$ whose dimension $\delta_{0} \geq 2$ changes to dimension $\delta_{1}>\delta_{0}$ at some random/unobservable time $\theta$ taking value 0 with probability $\pi \in[0,1]$ and being exponentially distributed with parameter $\lambda>0$ given that $\theta>0$. The problem is to detect the unknown time $\theta$ at which the dimension of $X$ changes as accurately as possible (neither too early nor too late). This problem belongs to the class of quickest detection problems as discussed in Section 1 above.

2. The observed process $X$ solves the stochastic differential equation:

$$
d X_{t}=\left[\mu_{0}\left(X_{t}\right)+I(t \geq \theta)\left(\mu_{1}\left(X_{t}\right)-\mu_{0}\left(X_{t}\right)\right)\right] d t+\sigma\left(X_{t}\right) d B_{t}
$$


driven by a standard Brownian motion $B$ under $\mathrm{P}_{\pi}$ specified below where we set

$$
\mu_{0}(x)=\frac{\delta_{0}-1}{2 x}, \quad \mu_{1}(x)=\frac{\delta_{1}-1}{2 x} \quad \text { and } \quad \sigma(x)=1
$$

for $x>0$. We assume throughout that $X$ starts at a strictly positive point and we will see below that this also yields solution when $X$ starts at zero. The unobservable time $\theta$ and the driving Brownian motion $B$ are assumed to be independent under $\mathrm{P}_{\pi}$ for $\pi \in[0,1]$.

3. Standard arguments imply that the previous setting can be realised on a probability space $\left(\Omega, \mathcal{F}, \mathrm{P}_{\pi}\right)$ with the probability measure $\mathrm{P}_{\pi}$ decomposed as follows:

$$
\mathrm{P}_{\pi}=\pi \mathrm{P}^{0}+(1-\pi) \int_{0}^{\infty} \lambda e^{-\lambda t} \mathrm{P}^{t} d t
$$

for $\pi \in[0,1]$ where $\mathrm{P}^{t}$ is the probability measure under which the observed process $X$ undergoes the change of dimension at time $t \in[0, \infty)$. The decomposition (3.3) expresses the fact that the unobservable time $\theta$ is a nonnegative random variable satisfying $\mathrm{P}_{\pi}(\theta=0)=\pi$ and $\mathrm{P}_{\pi}(\theta>t \mid \theta>0)=e^{-\lambda t}$ for $t>0$. Thus, $\mathrm{P}^{t}(X \in \cdot)=\mathrm{P}_{\pi}(X \in \cdot \mid \theta=t)$ is the probability law of a Bessel process whose dimension $\delta_{0} \geq 2$ changes to dimension $\delta_{1}>\delta_{0}$ at time $t \in[0, \infty)$. To remain consistent with this notation, we also denote by $\mathrm{P}^{\infty}$ the probability measure under which the observed process $X$ undergoes no change of its dimension. Thus, $\mathrm{P}^{\infty}(X \in \cdot)=\mathrm{P}_{\pi}(X \in \cdot \mid \theta=\infty)$ is the probability law of a Bessel process with dimension $\delta_{0} \geq 2$ at all times.

4. Being based upon continuous observation of $X$, the problem is to find a stopping time $\tau_{*}$ of $X$ [i.e., a stopping time with respect to the natural filtration $\mathcal{F}_{t}^{X}=\sigma\left(X_{s} \mid 0 \leq s \leq t\right)$ of $X$ for $\left.t \geq 0\right]$ that is "as close as possible" to the unknown time $\theta$. More precisely, the problem consists of computing the value function

$$
V(\pi)=\inf _{\tau}\left[\mathrm{P}_{\pi}(\tau<\theta)+c \mathrm{E}_{\pi}(\tau-\theta)^{+}\right]
$$

and finding the optimal stopping time $\tau_{*}$ at which the infimum in (3.4) is attained for $\pi \in[0,1]$ and $c>0$ given and fixed. Note in (3.4) that $\mathrm{P}_{\pi}(\tau<\theta)$ is the probability of the false alarm and $\mathrm{E}_{\pi}(\tau-\theta)^{+}$is the expected detection delay associated with a stopping time $\tau$ of $X$ for $\pi \in[0,1]$. Recall also that the expression on the right-hand side of (3.4) is the Lagrangian associated with the constrained problems as discussed in Section 1 above.

5. To tackle the optimal stopping problem (3.4), we consider the posterior probability distribution process $\Pi=\left(\Pi_{t}\right)_{t \geq 0}$ of $\theta$ given $X$ that is defined by

$$
\Pi_{t}=\mathrm{P}_{\pi}\left(\theta \leq t \mid \mathcal{F}_{t}^{X}\right)
$$

for $t \geq 0$. The right-hand side of (3.4) can be rewritten to read

$$
V(\pi)=\inf _{\tau} \mathrm{E}_{\pi}\left(1-\Pi_{\tau}+c \int_{0}^{\tau} \Pi_{t} d t\right)
$$


for $\pi \in[0,1]$. If the signal-to-noise ratio defined by

$$
\rho(x)=\frac{\mu_{1}(x)-\mu_{0}(x)}{\sigma(x)}
$$

is constant for $x>0$, then $\Pi$ is known to be a one-dimensional Markov (diffusion) process so that the optimal stopping problem (3.6) can be tackled using established techniques both in infinite and finite horizon (see [15], Section 22). Note that this is no longer the case in the setting of the present problem since from (3.2) we see that

$$
\rho(x)=\frac{\gamma}{x} \neq \text { constant }
$$

for $x>0$ where we set $\gamma=\left(\delta_{1}-\delta_{0}\right) / 2$.

6 . To connect the process $\Pi$ to the observed process $X$ we consider the likelihood ratio process $L=\left(L_{t}\right)_{t \geq 0}$ defined by

$$
L_{t}=\frac{d \mathrm{P}_{t}^{0}}{d \mathrm{P}_{t}^{\infty}}
$$

where $\mathrm{P}_{t}^{0}$ and $\mathrm{P}_{t}^{\infty}$ denote the restrictions of the probability measures $\mathrm{P}^{0}$ and $\mathrm{P}^{\infty}$ to $\mathcal{F}_{t}^{X}$ for $t \geq 0$. By the Girsanov theorem, one finds that

$$
L_{t}=\exp \left(\int_{0}^{t} \frac{\mu_{1}\left(X_{S}\right)-\mu_{0}\left(X_{S}\right)}{\sigma^{2}\left(X_{S}\right)} d X_{s}-\frac{1}{2} \int_{0}^{t} \frac{\mu_{1}^{2}\left(X_{s}\right)-\mu_{0}^{2}\left(X_{S}\right)}{\sigma^{2}\left(X_{S}\right)} d s\right)
$$

for $t \geq 0$. A direct calculation based on (3.3) shows that the posterior probability distribution ratio process $\Phi=\left(\Phi_{t}\right)_{t \geq 0}$ of $\theta$ given $X$ that is defined by

$$
\Phi_{t}=\frac{\Pi_{t}}{1-\Pi_{t}}
$$

can be expressed in terms of $L$ (and hence $X$ as well) as follows:

$$
\Phi_{t}=e^{\lambda t} L_{t}\left(\Phi_{0}+\lambda \int_{0}^{t} \frac{d s}{e^{\lambda s} L_{s}}\right)
$$

for $t \geq 0$ where $\Phi_{0}=\pi /(1-\pi)$. Note that $L_{t}$ in (3.10) is expressed in terms of a stochastic integral with respect to $X$ and as such is not an explicit functional of the observed sample path of $X$ up to time $t$. We will see in Section 6 below that such an explicit functional can be determined and that this issue is closely related to the parabolic nature of the underlying partial differential equation.

7. To derive stochastic differential equations for the posterior processes $\Pi$ and $\Phi$, one may apply Itô's formula in (3.10) to find that

$$
d L_{t}=\frac{\mu_{1}\left(X_{t}\right)-\mu_{0}\left(X_{t}\right)}{\sigma^{2}\left(X_{t}\right)} L_{t}\left[d X_{t}-\mu_{0}\left(X_{t}\right) d t\right]
$$


with $L_{0}=1$. Further applications of Itô's formula in (3.11) and (3.12) then show that

$$
\begin{aligned}
& d \Pi_{t}=\lambda\left(1-\Pi_{t}\right) d t+\rho\left(X_{t}\right) \Pi_{t}\left(1-\Pi_{t}\right) d \bar{B}_{t} \\
& d \Phi_{t}=\left[\lambda\left(1+\Phi_{t}\right)+\rho^{2}\left(X_{t}\right) \frac{\Phi_{t}^{2}}{1+\Phi_{t}}\right] d t+\rho\left(X_{t}\right) \Phi_{t} d \bar{B}_{t}
\end{aligned}
$$

upon noting that $X$ solves

$$
d X_{t}=\left[\mu_{0}\left(X_{t}\right)+\Pi_{t}\left(\mu_{1}\left(X_{t}\right)-\mu_{0}\left(X_{t}\right)\right)\right] d t+\sigma\left(X_{t}\right) d \bar{B}_{t},
$$

where $\bar{B}=\left(\bar{B}_{t}\right)_{t \geq 0}$ is the innovation process defined by

$$
\bar{B}_{t}=\int_{0}^{t} \frac{d X_{s}}{\sigma\left(X_{s}\right)}-\int_{0}^{t}\left[\frac{\mu_{0}\left(X_{s}\right)}{\sigma\left(X_{s}\right)}+\Pi_{s} \frac{\mu_{1}\left(X_{s}\right)-\mu_{0}\left(X_{s}\right)}{\sigma\left(X_{s}\right)}\right] d s
$$

for $t \geq 0$ from where we see by Lévy's characterisation theorem that $\bar{B}$ is a standard Brownian motion with respect to $\left(\mathcal{F}_{t}^{X}\right)_{t \geq 0}$ under $\mathrm{P}_{\pi}$ for $\pi \in[0,1]$.

8. From (3.14) and (3.15), it is evident that $\Pi$ and $\Phi$ cannot be Markov processes unless the signal-to-noise ratio $\rho$ defined in (3.7) is constant. If $\rho$ is not constant such as in (3.8) above, then one needs to look at (3.14) + (3.16) and $(3.15)+(3.16)$ as two systems of stochastic differential equations for the pairs of processes $(\Pi, X)$ and $(\Phi, X)$, respectively. It is well known (see, e.g., [17], pages $158-163)$ that when these systems have a unique weak solution then $(\Pi, X)$ and $(\Phi, X)$ are (time-homogeneous) strong Markov processes under $\mathrm{P}_{\pi}$ for $\pi \in[0,1]$. Recalling known sufficient conditions for the existence and uniqueness of weak solutions (see, e.g., [17], pages 166-173) we see that this is the case whenever $x \mapsto \mu_{0}(x), x \mapsto \mu_{1}(x)$ and $x \mapsto \sigma(x)$ are continuous with $\mu_{0}(x) \neq \mu_{1}(x)$ and $\sigma(x)>0$ for all $x$ in the state space of $X$ (possibly excluding entrance boundary points). Note that these conclusions are not confined to the setting of Bessel processes but hold generally in the quickest detection problems for diffusion processes $X$ solving (3.1) when the old drift $\mu_{0}$ is changing to the new drift $\mu_{1}$ at the random/unobservable time $\theta$.

9. The preceding considerations show that the optimal stopping problems (3.4) and (3.6) are inherently/fully two-dimensional with the pairs of processes $(\Pi, X)$ and $(\Phi, X)$ solving (3.14) + (3.16) and (3.15) + (3.16) being strong Markov when (3.8) holds. This fact makes the subsequent analysis of these problems more challenging than when the signal-to-noise ratio $\rho$ defined in (3.7) is constant. The analysis of (3.4) and (3.6) performed in [9] is based on the stochastic differential equations $(3.14)+(3.16)$ and $(3.15)+(3.16)$ under the probability measure $\mathrm{P}_{\pi}$ for $\pi \in[0,1]$. In this case, one sees that these two systems of stochastic differential equations are fully coupled [as both $\Pi$ and $X$ as well as $\Phi$ and $X$ enter both (3.14) and (3.16) as well as (3.15) and (3.16), respectively]. This makes the analy- 
sis of (3.4) and (3.6) more involved. In the next section, we will see that a change of measure argument simplifies the setting and decouples the systems (3.14) + (3.16) and (3.15) + (3.16) in the second equation so that the analysis of (3.4) and (3.6) becomes easier and more penetrating. This change of measure argument is not confined to the Bessel process setting and holds in general. Moreover, another major difficulty encountered in [9] is that both $\Pi$ and $X$ as well as $\Phi$ and $X$ enter the diffusion coefficient (3.15) and (3.16), respectively. This makes the use of comparison theorems for the systems of stochastic differential equations (3.14) + $(3.16)$ and $(3.15)+(3.16)$ more challenging. We will see in Section 7 below that time change arguments remove the dependence of the diffusion coefficient on the process $X$ in both systems so that known comparison theorems are applicable in this case. This will enable us in Section 8 below to derive monotonicity of the optimal stopping boundary that plays an important role in characterising the solution to the optimal stopping problems (3.4) and (3.6) above.

4. Measure change. In this section, we show that changing the measure $P_{\pi}$ for $\pi \in[0,1]$ to $\mathrm{P}^{\infty}$ in the optimal stopping problems (3.4) and (3.6) above provides crucial simplifications of the setting which makes the subsequent analysis possible. We first describe the rationale for the change of measure calculation and then furnish its formal verification in the proof of Lemma 1 below. Recalling that the systems of stochastic differential equations $(3.14)+(3.16)$ and $(3.15)+(3.16)$ are equivalent our focus in the sequel will be on the system $(3.15)+(3.16)$ for the pair of processes $(\Phi, X)$ after showing that this system takes a simpler form under the new measure $\mathrm{P}^{\infty}$. This is then followed by a reformulation of the optimal stopping problem (3.6) in terms of $(\Phi, X)$ under the new measure $\mathrm{P}^{\infty}$ in Proposition 2 below upon recalling that the optimal stopping problems (3.4) and (3.6) are equivalent.

1. To calculate the Radon-Nikodym derivative corresponding to the change of measure from $\mathrm{P}_{\pi}$ to $\mathrm{P}^{\infty}$ with $\pi \in[0,1]$ given and fixed, we may recall that if $Q=\alpha Q_{1}+(1-\alpha) Q_{2}$ where $Q_{1}$ and $Q_{2}$ are probability measures on $(\Omega, \mathcal{F})$ and $\alpha \in[0,1]$ then

$$
\mathrm{E}_{Q}(X \mid \mathcal{G})=\alpha \mathrm{E}_{Q_{1}}(X \mid \mathcal{G}) \frac{d Q_{1}^{\mathcal{G}}}{d Q^{\mathcal{G}}}+(1-\alpha) \mathrm{E}_{Q_{2}}(X \mid \mathcal{G}) \frac{d Q_{2}^{\mathcal{G}}}{d Q^{\mathcal{G}}}
$$

for any (integrable) random variable $X$ on $(\Omega, \mathcal{F})$ and any $\sigma$-algebra $\mathcal{G} \subseteq \mathcal{F}$ where $Q_{i}^{\mathcal{G}}$ and $Q^{\mathcal{G}}$ denote the restrictions of $Q_{i}$ and $Q$ to $\mathcal{G}$ for $i=1,2$, respectively. Clearly, (4.1) follows by a direct verification and the argument extends to more general convex combinations of probability measures including their integrals as limiting cases. With this argument in place, we can then return to the setting described in Section 3 above and proceed as follows. 
2. Denoting by $\mathrm{P}_{t}^{s}$ and $\mathrm{P}_{\pi, t}$ the restrictions of measures $\mathrm{P}^{s}$ and $\mathrm{P}_{\pi}$ to $\mathcal{F}_{t}^{X}$ for $s \geq 0$ and $t \geq 0$, we find by (3.3) using the extended version of (4.1) above that

$1-\Pi_{t}=1-\mathrm{P}_{\pi}\left(\theta \leq t \mid \mathcal{F}_{t}^{X}\right)=\mathrm{P}_{\pi}\left(\theta>t \mid \mathcal{F}_{t}^{X}\right)$

$$
\begin{aligned}
& =\pi \mathrm{P}^{0}\left(\theta>t \mid \mathcal{F}_{t}^{X}\right) \frac{d \mathrm{P}_{t}^{0}}{d \mathrm{P}_{\pi, t}}+(1-\pi) \int_{0}^{\infty} \lambda e^{-\lambda s} \mathrm{P}^{s}\left(\theta>t \mid \mathcal{F}_{t}^{X}\right) \frac{d \mathrm{P}_{t}^{s}}{d \mathrm{P}_{\pi, t}} d s \\
& =(1-\pi) \int_{t}^{\infty} \lambda e^{-\lambda s} \frac{d \mathrm{P}_{t}^{\infty}}{d \mathrm{P}_{\pi, t}} d s=(1-\pi) e^{-\lambda t} \frac{d \mathrm{P}_{t}^{\infty}}{d \mathrm{P}_{\pi, t}},
\end{aligned}
$$

where we use that $\mathrm{P}^{0}\left(\theta>t \mid \mathcal{F}_{t}^{X}\right)$ equals 0 and $\mathrm{P}^{s}\left(\theta>t \mid \mathcal{F}_{t}^{X}\right)$ equals 1 if $s>t$ and 0 if $s \leq t$, and we also use that $d \mathrm{P}_{t}^{s} / d \mathrm{P}_{\pi, t}=d \mathrm{P}_{t}^{\infty} / d \mathrm{P}_{\pi, t}$ for $s>t$. From (4.2), we find that

$$
\frac{d \mathrm{P}_{\pi, t}}{d \mathrm{P}_{t}^{\infty}}=e^{-\lambda t} \frac{1-\pi}{1-\Pi_{t}}
$$

for $t \in[0, \infty)$ and $\pi \in[0,1)$. Using (3.11) and (3.12), it is easily seen that the right-hand side in (4.3) tends to $L_{t}$ as $\pi \uparrow 1$. Given that $\mathrm{P}_{1, t}$ coincides with $\mathrm{P}_{t}^{0}$ we see that this consequence is consistent with (3.9) above.

3. We now formally verify that the identity (4.3) extends to stopping times $\tau$ of $X$ as well. In this case we let $\mathrm{P}_{\tau}^{\infty}$ and $\mathrm{P}_{\pi, \tau}$ denote the restrictions of measures $\mathrm{P}^{\infty}$ and $\mathrm{P}_{\pi}$ to $\mathcal{F}_{\tau}^{X}$ for $\pi \in[0,1)$, respectively.

LEMMA 1. The following identity holds:

$$
\frac{d \mathrm{P}_{\pi, \tau}}{d \mathrm{P}_{\tau}^{\infty}}=e^{-\lambda \tau} \frac{1-\pi}{1-\Pi_{\tau}}
$$

for all stopping times $\tau$ of $X$ and all $\pi \in[0,1)$.

PROOF. It is enough to show that

$$
\frac{d \mathrm{P}_{\tau}^{\infty}}{d \mathrm{P}_{\pi, \tau}}=e^{\lambda \tau} \frac{1-\Pi_{\tau}}{1-\pi}
$$

on $\mathcal{F}_{\tau}^{X}$ with $\tau$ and $\pi$ as above given and fixed. For this, take $F \in \mathcal{F}_{\tau}^{X}$. Then

$$
\begin{aligned}
\int_{F} e^{\lambda \tau} & \frac{1-\Pi_{\tau}}{1-\pi} d \mathrm{P}_{\pi, \tau} \\
& =\frac{1}{1-\pi} \mathrm{E}_{\pi}\left[1_{F} e^{\lambda \tau}\left(1-\Pi_{\tau}\right)\right]=\frac{1}{1-\pi} \mathrm{E}_{\pi}\left[1_{F} e^{\lambda \tau} \mathrm{P}_{\pi}\left(\theta>\tau \mid \mathcal{F}_{\tau}^{X}\right)\right] \\
& =\frac{1}{1-\pi} \mathrm{E}_{\pi}\left[\mathrm{E}_{\pi}\left(1_{F} e^{\lambda \tau} I(\theta>\tau) \mid \mathcal{F}_{\tau}^{X}\right)\right]=\frac{1}{1-\pi} \mathrm{E}_{\pi}\left[1_{F} e^{\lambda \tau} I(\theta>\tau)\right] \\
\text { (4.6) } \quad & =\frac{1}{1-\pi}\left(\pi \mathrm{E}^{0}\left[1_{F} e^{\lambda \tau} I(\theta>\tau)\right]\right.
\end{aligned}
$$




$$
\begin{aligned}
& \left.+(1-\pi) \int_{0}^{\infty} \lambda e^{-\lambda t} \mathrm{E}^{t}\left[1_{F} e^{\lambda \tau} I(\theta>\tau)\right] d t\right) \\
= & \int_{0}^{\infty} \lambda e^{-\lambda t} \mathrm{E}^{t}\left[1_{F} e^{\lambda \tau} I(t>\tau)\right] d t=\int_{0}^{\infty} \lambda e^{-\lambda t} \mathrm{E}^{\infty}\left[1_{F} e^{\lambda \tau} I(t>\tau)\right] d t \\
= & \mathrm{E}^{\infty}\left[1_{F} e^{\lambda \tau} \int_{\tau}^{\infty} \lambda e^{-\lambda t} d t\right]=\mathrm{P}_{\tau}^{\infty}(F)
\end{aligned}
$$

as needed to verify (4.5) and the proof is complete.

4. From (3.15) and (3.17), we see that the stochastic differential equations for $(\Phi, X)$ under the measure $\mathrm{P}^{\infty}$ simplify to read as follows:

$$
\begin{aligned}
& d \Phi_{t}=\lambda\left(1+\Phi_{t}\right) d t+\rho\left(X_{t}\right) \Phi_{t} d B_{t}, \\
& d X_{t}=\mu_{0}\left(X_{t}\right) d t+\sigma\left(X_{t}\right) d B_{t},
\end{aligned}
$$

where (4.8) follows from (3.1) upon recalling that $\theta$ formally equals $\infty$ under $\mathrm{P}^{\infty}$. Recall that $\rho$ in (4.7) is given by (3.8) above, and $\mu_{0}$ and $\sigma$ in (4.8) are given in (3.2) above. The stochastic differential equations (4.7) $+(4.8)$ also hold in general under $\mathrm{P}^{\infty}$ whenever $\mu_{0} \neq \mu_{1}$ and $\sigma>0$ are continuous and for the reasons stated at the end of Section 3 in this case we know that $(\Phi, X)$ is a strong Markov process under $\mathrm{P}^{\infty}$. Note also from (3.13) that the stochastic differential equation for $L$ under $\mathrm{P}^{\infty}$ simplifies to read as follows:

$$
d L_{t}=\rho\left(X_{t}\right) L_{t} d B_{t}
$$

so that [as it is also seen from (3.10) above] we have

$$
L_{t}=\exp \left(\int_{0}^{t} \rho\left(X_{s}\right) d B_{s}-\frac{1}{2} \int_{0}^{t} \rho^{2}\left(X_{s}\right) d s\right)
$$

for $t \geq 0$. The stochastic differential equation (3.14) for the process $\Pi$ takes a more complicated form under the measure $\mathrm{P}^{\infty}$ and given that this equation is equivalent to (4.7) due to (3.11) we will not state it explicitly. Thus, our focus in the sequel will be on the system $(4.7)+(4.8)$ for the pair of processes $(\Phi, X)$ under the measure $\mathrm{P}^{\infty}$.

5. We now show that the optimal stopping problem (3.6) admits a transparent reformulation under the measure $\mathrm{P}^{\infty}$ in terms of the process $\Phi$ solving (4.7) with (4.8). Recall that $\Phi$ starts at $\Phi_{0}=\pi /(1-\pi)$ and this dependence on the initial point will be indicated by a superscript to $\Phi$ when needed.

Proposition 2. The value function $V$ from (3.6) satisfies the identity

$$
V(\pi)=(1-\pi)[1+c \hat{V}(\pi)],
$$

where the value function $\hat{V}$ is given by

$$
\hat{V}(\pi)=\inf _{\tau} \mathrm{E}^{\infty}\left[\int_{0}^{\tau} e^{-\lambda t}\left(\Phi_{t}^{\pi /(1-\pi)}-\frac{\lambda}{c}\right) d t\right]
$$

for $\pi \in[0,1)$ and the infimum in (4.12) is taken over all stopping times $\tau$ of $X$. 
PRoOF. With $\pi \in[0,1)$ given and fixed, and dropping the superscript from $\Phi$ in the sequel for simplicity, by standard localisation arguments it is enough to show that

$$
\begin{aligned}
\mathrm{E}_{\pi}\left(1-\Pi_{\tau}+c \int_{0}^{\tau} \Pi_{t} d t\right) \\
\quad=(1-\pi)\left(1+c \mathrm{E}^{\infty}\left[\int_{0}^{\tau} e^{-\lambda t}\left(\Phi_{t}-\frac{\lambda}{c}\right) d t\right]\right)
\end{aligned}
$$

for all bounded stopping times $\tau$ of $X$ such that $\Pi^{\tau}=\left(\Pi_{t \wedge \tau}\right)_{t \geq 0}$ [and hence $\Phi^{\tau}=$ $\left(\Phi_{t \wedge \tau}\right)_{t \geq 0}$ as well] and $\rho(X)^{\tau}=\left(\rho\left(X_{t \wedge \tau}\right)\right)_{t \geq 0}$ are bounded (recall that $X$ starts at a strictly positive point and stays away from 0 at all times). Let such a stopping time $\tau$ be given and fixed in the sequel. From (3.14), we find by the optional sampling theorem that

$$
\mathrm{E}_{\pi}\left(\int_{0}^{\tau} \Pi_{t} d t\right)=\frac{1}{\lambda}\left[\pi+\lambda \mathrm{E}_{\pi}(\tau)-\mathrm{E}_{\pi}\left(\Pi_{\tau}\right)\right] .
$$

Inserting this expression into the left-hand side of (4.13) and using Lemma 1 we get

$$
\begin{aligned}
\mathrm{E}_{\pi}( & \left.-\Pi_{\tau}+c \int_{0}^{\tau} \Pi_{t} d t\right) \\
& =\mathrm{E}_{\pi}\left[1-\left(1+\frac{c}{\lambda}\right) \Pi_{\tau}+c \tau\right]+\frac{c}{\lambda} \pi \\
& =\frac{\lambda+c}{\lambda} \mathrm{E}^{\infty}\left[e^{-\lambda \tau} \frac{1-\pi}{1-\Pi_{\tau}}\left(1-\Pi_{\tau}+\frac{\lambda c}{\lambda+c} \tau\right)\right]-\frac{c}{\lambda}(1-\pi) \\
& =(1-\pi)\left(1+\frac{\lambda+c}{\lambda} \mathrm{E}^{\infty}\left[-\lambda \int_{0}^{\tau} e^{-\lambda t} d t+\frac{\lambda c}{\lambda+c} \tau e^{-\lambda \tau}\left(1+\Phi_{\tau}\right)\right]\right),
\end{aligned}
$$

where we use that $1 /\left(1-\Pi_{t}\right)=1+\Phi_{t}$ for $t \geq 0$. Integration by parts using (4.7) shows that

$$
\begin{aligned}
M_{t} & :=e^{-\lambda t}\left(1+\Phi_{t}\right)=1+\Phi_{0}-\lambda \int_{0}^{t} e^{-\lambda s}\left(1+\Phi_{s}\right) d s+\int_{0}^{t} e^{-\lambda s} d \Phi_{s} \\
& =1+\Phi_{0}+\int_{0}^{t} e^{-\lambda s} \rho\left(X_{s}\right) \Phi_{s} d B_{s}
\end{aligned}
$$

is a continuous local martingale under $\mathrm{P}^{\infty}$ for $t \geq 0$. Another integration by parts yields

$$
t M_{t}=\int_{0}^{t} M_{s} d s+\int_{0}^{t} s d M_{s},
$$

where the final integral defines another continuous local martingale under $\mathrm{P}^{\infty}$ for $t \geq 0$. Recalling the definition of $\tau$ it is easily verified that the optional sampling 
theorem is applicable to that local martingale stopped at $\tau$ and in this way we get

$$
\begin{aligned}
\mathrm{E}^{\infty}\left[\tau e^{-\lambda \tau}\left(1+\Phi_{\tau}\right)\right] & =\mathrm{E}^{\infty}\left(\tau M_{\tau}\right)=\mathrm{E}^{\infty}\left(\int_{0}^{\tau} M_{t} d t\right) \\
& =\mathrm{E}^{\infty}\left[\int_{0}^{\tau} e^{-\lambda t}\left(1+\Phi_{t}\right) d t\right]
\end{aligned}
$$

Inserting this expression in (4.15), we find that (4.13) is satisfied and the proof is complete.

6. Note that the identities (4.11) and (4.12) are not confined to the setting of Bessel processes but hold generally in the quickest detection problems for diffusion processes $X$ solving (3.1) when the old drift $\mu_{0}$ is changing to the new drift $\mu_{1}$ at the random/unobservable time $\theta$. If $\rho$ is not constant such as in (3.8) above, then to tackle the resulting optimal stopping problem (4.12) for the strong Markov process $(\Phi, X)$ solving $(4.7)+(4.8)$ we will enable $(\Phi, X)$ to start at any point $(\varphi, x)$ in $[0, \infty) \times(0, \infty)$ under the probability measure $\mathrm{P}_{\varphi, x}^{\infty}$ so that the optimal stopping problem (4.12) extends as follows:

$$
\hat{V}(\varphi, x)=\inf _{\tau} \mathrm{E}_{\varphi, x}^{\infty}\left[\int_{0}^{\tau} e^{-\lambda t}\left(\Phi_{t}-\frac{\lambda}{c}\right) d t\right]
$$

for $(\varphi, x) \in[0, \infty) \times(0, \infty)$ with $\mathrm{P}_{\varphi, x}^{\infty}\left(\left(\Phi_{0}, X_{0}\right)=(\varphi, x)\right)=1$ where the infimum in (4.19) is taken over all stopping times $\tau$ of $(\Phi, X)$. In this way, we have reduced the initial quickest detection problems (3.4) and (3.6) to the optimal stopping problem (4.19) for the strong Markov process $(\Phi, X)$ solving the system of stochastic differential equations

$$
\begin{aligned}
& d \Phi_{t}=\lambda\left(1+\Phi_{t}\right) d t+\gamma \frac{\Phi_{t}}{X_{t}} d B_{t}, \\
& d X_{t}=\frac{\delta_{0}-1}{2 X_{t}} d t+d B_{t}
\end{aligned}
$$

under the measure $\mathrm{P}_{\varphi, x}^{\infty}$ with $(\varphi, x) \in[0, \infty) \times(0, \infty)$ where we recall that $\gamma=\left(\delta_{1}-\delta_{0}\right) / 2$. Note that this optimal stopping problem is inherently/fully twodimensional.

5. Mayer formulation. The optimal stopping problem (4.19) is Lagrange formulated. In this section, we derive its Mayer reformulation which is helpful in the subsequent analysis. We will first present a stochastic derivation of the Mayer formulation in the proof below and then indicate how this also follows more directly by verifying that the candidate function (once it is given or found) solves a killed infinitesimal generator equation for $(\Phi, X)$. 
Proposition 3. The value function $\hat{V}$ from (4.19) satisfies the identity

$$
\hat{V}(\varphi, x)=\frac{1}{\delta_{1}}\left[\bar{V}(\varphi, x)-(1+\varphi) x^{2}-\alpha\right]
$$

where the value function $\bar{V}$ is given by

$$
\bar{V}(\varphi, x)=\inf _{\tau} \mathrm{E}_{\varphi, x}^{\infty}\left(e^{-\lambda \tau}\left[\left(1+\Phi_{\tau}\right) X_{\tau}^{2}+\alpha\right]\right)
$$

for $(\varphi, x) \in[0, \infty) \times(0, \infty)$ with $\alpha=\delta_{0} / \lambda+\delta_{1} / c$ and the infimum in (5.2) is taken over all stopping times $\tau$ of $(\Phi, X)$.

Proof. Set $Y=X^{2}$ and $y=x^{2}$ for $x>0$. With $(\varphi, x) \in[0, \infty) \times(0, \infty)$ given and fixed, and dropping the subscript from $\mathrm{E}^{\infty}$ in the sequel for simplicity, by standard localisation arguments it is enough to show that

$$
\begin{aligned}
\mathrm{E}^{\infty}[ & \left.\int_{0}^{\tau} e^{-\lambda t}\left(\Phi_{t}-\frac{\lambda}{c}\right) d t\right] \\
& =\frac{1}{\delta_{1}}\left[\mathrm{E}^{\infty}\left(e^{-\lambda \tau}\left[\left(1+\Phi_{\tau}\right) Y_{\tau}+\alpha\right]\right)-\left(1+\Phi_{0}\right) Y_{0}-\alpha\right]
\end{aligned}
$$

for all bounded stopping times $\tau$ of $(\Phi, X)$ such that $\Phi^{\tau}=\left(\Phi_{t \wedge \tau}\right)_{t \geq 0}$ and $Y^{\tau}=$ $\left(Y_{t \wedge \tau}\right)_{t \geq 0}$ are bounded. Let such a stopping time $\tau$ be given and fixed in the sequel. From (4.21), we find by Itô's formula that $Y$ solves

$$
d Y_{t}=\delta_{0} d t+2 \sqrt{Y_{t}} d B_{t} .
$$

Using (4.20) and (5.4), we find by another application of Itô's formula that

$$
\Phi_{t} Y_{t}=\Phi_{0} Y_{0}+\int_{0}^{t}\left(\delta_{1} \Phi_{s}+\lambda\left(1+\Phi_{s}\right) Y_{s}\right) d s+\int_{0}^{t}(2+\gamma) \Phi_{s} \sqrt{Y_{s}} d B_{s}
$$

for $t \geq 0$. Integration by parts using (5.5) then yields

$$
\begin{aligned}
e^{-\lambda t} \Phi_{t} Y_{t}= & \Phi_{0} Y_{0}+\delta_{1} \int_{0}^{t} e^{-\lambda s} \Phi_{s} d s+\lambda \int_{0}^{t} e^{-\lambda s} Y_{s} d s \\
& +(2+\gamma) \int_{0}^{t} e^{-\lambda s} \Phi_{s} \sqrt{Y_{s}} d B_{s},
\end{aligned}
$$

where the final integral defines a continuous local martingale under $\mathrm{P}^{\infty}$ for $t \geq 0$. Recalling the definition of $\tau$ it is easily verified that the optional sampling theorem is applicable to that local martingale stopped at $\tau$ and in this way we get

$$
\begin{aligned}
& \mathrm{E}^{\infty}\left(e^{-\lambda \tau} \Phi_{\tau} Y_{\tau}\right) \\
& \quad=\Phi_{0} Y_{0}+\delta_{1} \mathrm{E}^{\infty}\left(\int_{0}^{\tau} e^{-\lambda s} \Phi_{s} d s\right)+\lambda \mathrm{E}^{\infty}\left(\int_{0}^{\tau} e^{-\lambda s} Y_{s} d s\right) .
\end{aligned}
$$


To calculate the final expectation above another integration by parts combined with (5.4) yields

$$
e^{-\lambda t} Y_{t}=Y_{0}-\lambda \int_{0}^{t} e^{-\lambda s} Y_{s} d s+\delta_{0} \int_{0}^{t} e^{-\lambda s} d s+2 \int_{0}^{t} e^{-\lambda s} \sqrt{Y_{s}} d B_{s},
$$

where the final integral defines a continuous local martingale under $\mathrm{P}^{\infty}$ for $t \geq 0$. For the same reasons as above, the optional sampling theorem is applicable to that local martingale stopped at $\tau$ and in this way we get

$$
\mathrm{E}^{\infty}\left(e^{-\lambda \tau} Y_{\tau}\right)=Y_{0}-\lambda \mathrm{E}^{\infty}\left(\int_{0}^{\tau} e^{-\lambda s} Y_{s} d s\right)+\delta_{0} \mathrm{E}^{\infty}\left(\int_{0}^{\tau} e^{-\lambda s} d s\right) .
$$

Inserting (5.9) into (5.7), we find that

$$
\begin{aligned}
\mathrm{E}^{\infty}\left(\int_{0}^{\tau} e^{-\lambda t} \Phi_{t} d t\right)= & \frac{1}{\delta_{1}}\left[\mathrm{E}^{\infty}\left(e^{-\lambda \tau} \Phi_{\tau} Y_{\tau}\right)+\mathrm{E}^{\infty}\left(e^{-\lambda \tau} Y_{\tau}\right)\right. \\
& \left.-\delta_{0} \mathrm{E}^{\infty}\left(\int_{0}^{\tau} e^{-\lambda s} d s\right)-\Phi_{0} Y_{0}-Y_{0}\right] .
\end{aligned}
$$

Subtracting $(\lambda / c) \mathrm{E}^{\infty}\left(\int_{0}^{\tau} e^{-\lambda s} d s\right)$ from both sides in (5.10) and using that

$$
\begin{aligned}
-\frac{\lambda}{c} \mathrm{E}^{\infty} & \left(\int_{0}^{\tau} e^{-\lambda s} d s\right)-\frac{\delta_{0}}{\delta_{1}} \mathrm{E}^{\infty}\left(\int_{0}^{\tau} e^{-\lambda s} d s\right) \\
& =\frac{1}{\delta_{1}}\left[\left(\frac{\delta_{1}}{c}+\frac{\delta_{0}}{\lambda}\right) \mathrm{E}^{\infty}\left(e^{-\lambda \tau}\right)-\left(\frac{\delta_{1}}{c}+\frac{\delta_{0}}{\lambda}\right)\right]
\end{aligned}
$$

we see that (5.3) holds as claimed and the proof is complete.

1. Defining a function $M$ by setting

$$
M(\varphi, x)=\frac{1}{\delta_{1}}\left[(1+\varphi) x^{2}+\alpha\right]
$$

we see from Proposition 3 that the optimal stopping problem (4.19) is equivalent to the optimal stopping problem defined by

$$
\check{V}(\varphi, x)=\inf _{\tau} \mathrm{E}_{\varphi, x}^{\infty}\left[e^{-\lambda \tau} M\left(\Phi_{\tau}, X_{\tau}\right)\right]
$$

for $(\varphi, x) \in[0, \infty) \times(0, \infty)$ where the infimum in (5.13) is taken over all stopping times $\tau$ of $(\Phi, X)$. The optimal stopping problem (5.13) is Mayer formulated. From (5.1), we see that

$$
\check{V}(\varphi, x)=M(\varphi, x)+\hat{V}(\varphi, x)
$$

for all $(\varphi, x) \in[0, \infty) \times(0, \infty)$. The identity (5.14) can also be obtained by noting that $M$ from (5.12) is a particular solution to

$$
\mathbb{L}_{\Phi, X} M-\lambda M=H
$$


on $[0, \infty) \times(0, \infty)$ where we set

$$
H(\varphi, x)=\varphi-\frac{\lambda}{c}
$$

for $(\varphi, x) \in[0, \infty) \times(0, \infty)$ and from $(4.20)+(4.21)$ we see that the infinitesimal generator $\mathbb{L}_{\Phi, X}$ of $(\Phi, X)$ is given by

$$
\mathbb{L}_{\Phi, X}=\lambda(1+\varphi) \partial_{\varphi}+\frac{\delta_{0}-1}{2 x} \partial_{x}+\gamma \frac{\varphi}{x} \partial_{\varphi x}+\frac{\gamma^{2}}{2} \frac{\varphi^{2}}{x^{2}} \partial_{\varphi \varphi}+\frac{1}{2} \partial_{x x} .
$$

Applying Itô's formula to $M(\Phi, X)$ and using the optional sampling theorem, we get

$$
\mathrm{E}_{\varphi, x}^{\infty}\left[e^{-\lambda \tau} M\left(\Phi_{\tau}, X_{\tau}\right)\right]=M(\varphi, x)+\mathrm{E}_{\varphi, x}^{\infty}\left[\int_{0}^{\tau} e^{-\lambda t} H\left(\Phi_{t}, X_{t}\right) d t\right]
$$

for all stopping times $\tau$ of $(\Phi, X)$ as in the proof of Proposition 3 from where we obtain (5.14) by a simple limiting argument as claimed.

2. Note that the Mayer reformulation (5.1) + (5.2) of the optimal stopping problem (4.19) is specific to the setting of Bessel processes. To find a Mayer reformulation of (4.19) in the quickest detection problems for diffusion processes $X$ solving (3.1) when the old drift $\mu_{0}$ is changing to the new drift $\mu_{1}$ at the random/unobservable time $\theta$, one needs to find a particular solution $M$ to equation (5.15) where the infinitesimal generator $\mathbb{L}_{\Phi, X}$ of $(\Phi, X)$ is given by

$$
\begin{aligned}
\mathbb{L}_{\Phi, X}= & \lambda(1+\varphi) \partial_{\varphi}+\mu_{0}(x) \partial_{x}+\varphi \rho(x) \sigma(x) \partial_{\varphi x} \\
& +\frac{1}{2} \varphi^{2} \rho^{2}(x) \partial_{\varphi \varphi}+\frac{1}{2} \sigma^{2}(x) \partial_{x x}
\end{aligned}
$$

and $\rho$ is given by (3.7) above. Note that such explicit solutions may not be available in general. Note also that if $\rho$ is constant then one needs to look for a solution $M$ to (5.15) that is a function of $\varphi$ only since in this case $\Phi$ is a one-dimensional strong Markov process.

6. Reduction to canonical PDE. Recall that we have reduced the initial quickest detection problems (3.4) and (3.6) to the optimal stopping problem (4.19) for the strong Markov process $(\Phi, X)$ solving $(4.7)+(4.8)$ which in the setting of Bessel processes becomes $(4.20)+(4.21)$. It follows in particular that the process $(\Phi, X)$ forms a sufficient statistic in the quickest detection problems (3.4) and (3.6). This is a consequence of the fact (which is formally verified in Section 8 below) that the first entry time of $(\Phi, X)$ to the stopping set $D$ is an optimal stopping time in (4.19), and hence in (3.4) and (3.6) as well. It is therefore of theoretical and practical importance to understand/describe how $\Phi$ is being formed through the observation of $X$.

On closer look, we see from (3.10) and (3.12) that $\Phi_{t}$ is expressed in terms of a stochastic integral with respect to $X$ and as such is not an explicit functional of 
the observed sample path of $X$ up to time $t$ in $[0, \infty)$. In this section, we show that such a functional can be determined explicitly. This is first done in the proof below using probabilistic arguments. It enables us to rewrite the optimal stopping problem (4.19) in terms of another strong Markov process $(U, \Phi)$ where the process $U$ is of bounded variation. We then show using analytic arguments that the passage from $(\Phi, X)$ to $(U, \Phi)$ is equivalent to the standard reduction of the parabolic equation $\mathbb{L}_{\Phi, X}$ to its canonical form $\mathbb{L}_{U, \Phi}$ where $\mathbb{L}_{\Phi, X}$ and $\mathbb{L}_{U, \Phi}$ denote the infinitesimal generators of $(\Phi, X)$ to $(U, \Phi)$, respectively. Note that these facts are not confined to the setting of Bessel process but hold generally in the quickest detection problems for diffusion processes $X$ solving (3.1) when the old drift $\mu_{0}$ is changing to the new dirft $\mu_{1}$ at the random/unobservable time $\theta$.

Although the subsequent analysis of Bessel processes will be largely performed using the process $(\Phi, X)$, we will also see that reduction to the process $(U, \Phi)$ has some advantages in the sequel when it comes to proving continuity of the optimal stopping boundary (Section 12), for example, as well as exploiting the parabolic (evolutionary) nature of the underlying partial differential equation in a simplifying/powerful manner (Section 14).

1. To formulate the next result, we assume that $x \mapsto \rho(x) / \sigma(x)$ is continuously differentiable in addition to $x \mapsto \mu_{0}(x), x \mapsto \mu_{1}(x)$ and $x \mapsto \sigma(x)$ being continuous with $\mu_{0}(x) \neq \mu_{1}(x)$ and $\sigma(x)>0$ for all $x$ in the state space of $X$ (possibly excluding entrance boundary points). Note that these assumptions are satisfied in the case of (3.2) above.

Proposition 4. Let the functions $F$ and $K$ be defined by

$$
\begin{aligned}
F(x) & =\int_{1}^{x} \frac{\rho(y)}{\sigma(y)} d y, \\
K & =\frac{1}{2}\left[\sigma^{2} F^{\prime \prime}+\left(\mu_{1}+\mu_{0}\right) F^{\prime}\right]=\frac{1}{2}\left[\sigma^{2}\left(\frac{\rho}{\sigma}\right)^{\prime}+\left(\mu_{1}+\mu_{0}\right)\left(\frac{\rho}{\sigma}\right)\right]
\end{aligned}
$$

for $x$ in the state space of $X$. Then the following identity holds:

$$
e^{F\left(X_{t}\right)}=\Phi_{t} e^{U_{t}},
$$

where the process $U=\left(U_{t}\right)_{t \geq 0}$ is defined by

$$
U_{t}=U_{0}+\int_{0}^{t}\left[K\left(F^{-1}\left(\log \Phi_{s}+U_{s}\right)\right)-\lambda \frac{1+\Phi_{s}}{\Phi_{s}}\right] d s
$$

for $t \geq 0$. In particular, the process $(U, \Phi)$ solving

$$
\begin{aligned}
& d U_{t}=\left[K\left(F^{-1}\left(\log \Phi_{t}+U_{t}\right)\right)-\lambda \frac{1+\Phi_{t}}{\Phi_{t}}\right] d t, \\
& d \Phi_{t}=\lambda\left(1+\Phi_{t}\right) d t+\rho\left(F^{-1}\left(\log \Phi_{t}+U_{t}\right)\right) \Phi_{t} d B_{t}
\end{aligned}
$$

is a (time-homogeneous) strong Markov process under $\mathrm{P}_{u, \varphi}^{\infty}$ with $\mathrm{P}_{u, \varphi}^{\infty}\left(\left(U_{0}, \Phi_{0}\right)=\right.$ $(u, \varphi))=1$ for $(u, \varphi) \in \mathbb{R} \times[0, \infty)$. 
ProOF. From (3.12), we see that

$$
\log \Phi_{t}=\lambda t+\log L_{t}+\log \left(\Phi_{0}+\lambda \int_{0}^{t} \frac{d s}{e^{\lambda s} L_{s}}\right)
$$

for $t \geq 0$. From (3.10), we find by Itô's formula using (6.1) and (6.2) that

$$
\begin{aligned}
\log L_{t}= & \int_{0}^{t}\left(\frac{\rho}{\sigma}\right)\left(X_{s}\right) d X_{s}-\frac{1}{2} \int_{0}^{t}\left(\mu_{1}+\mu_{0}\right)\left(\frac{\rho}{\sigma}\right)\left(X_{s}\right) d s \\
= & F\left(X_{t}\right)-F\left(X_{0}\right)-\frac{1}{2} \int_{0}^{t} F^{\prime \prime}\left(X_{s}\right) d\langle X, X\rangle_{s} \\
& -\frac{1}{2} \int_{0}^{t}\left(\mu_{1}+\mu_{0}\right)\left(\frac{\rho}{\sigma}\right)\left(X_{s}\right) d s \\
= & F\left(X_{t}\right)-F\left(X_{0}\right)-\int_{0}^{t} K\left(X_{S}\right) d s
\end{aligned}
$$

for $t \geq 0$. Moreover, integrating by parts we find that

$$
\log \left(\Phi_{0}+\lambda \int_{0}^{t} \frac{d s}{e^{\lambda s} L_{s}}\right)=\log \Phi_{0}+\lambda \int_{0}^{t} \frac{d s}{\Phi_{s}}
$$

for $t \geq 0$. Inserting (6.8) and (6.9) into (6.7), we obtain

$$
\log \Phi_{t}=F\left(X_{t}\right)-F\left(X_{0}\right)+\log \Phi_{0}+\int_{0}^{t}\left[\lambda\left(1+\frac{1}{\Phi_{s}}\right)-K\left(X_{s}\right)\right] d s
$$

for $t \geq 0$. From (6.10), we see that

$$
F\left(X_{t}\right)=\log \Phi_{t}+U_{t},
$$

where $U_{t}$ is given by the following expression:

$$
U_{t}=U_{0}+\int_{0}^{t}\left[K\left(X_{S}\right)-\lambda\left(1+\frac{1}{\Phi_{s}}\right)\right] d s
$$

for $t \geq 0$ with $U_{0}=F\left(X_{0}\right)-\log \Phi_{0}$. Applying the exponential function on both sides of (6.11), we see that (6.3) holds. From (6.11), we also see that $X_{s}=$ $F^{-1}\left(\log \Phi_{s}+U_{s}\right)$ for $s \in[0, t]$. Inserting this expression back into (6.12), we obtain (6.4). From (6.4), we see that (6.5) holds and (6.6) follows likewise from (4.7) above. Recalling known sufficient conditions (see, e.g., [17], pages 166-173) we see that the system of stochastic differential equations $(6.5)+(6.6)$ has a unique weak solution, and hence by the well-known result (see, e.g., [17], pages 158-163) we can conclude that $(U, \Phi)$ is a (time-homogeneous) strong Markov process un$\operatorname{der} \mathrm{P}_{u, \varphi}^{\infty}$ for $(u, \varphi) \in \mathbb{R} \times[0, \infty)$ as claimed. This completes the proof.

2. We now specialise the general result of Proposition 4 to the case of Bessel processes when the identities (3.2) above are satisfied. 
COROLlary 5. If $X$ solves (3.1) with (3.2), then

$$
F(x)=\log \left(x^{\gamma}\right) \quad \text { and } \quad K(x)=\frac{\beta}{x^{2}}
$$

for $x>0$ where $\gamma=\left(\delta_{1}-\delta_{0}\right) / 2$ and $\beta=\left(\delta_{1}-\delta_{0}\right)\left(\delta_{1}+\delta_{0}-4\right) / 8$. We thus have

$$
X_{t}^{\gamma}=\Phi_{t} e^{U_{t}}
$$

where the process $U=\left(U_{t}\right)_{t \geq 0}$ is defined by

$$
U_{t}=U_{0}+\int_{0}^{t}\left[\frac{\beta}{\Phi_{s}^{2 / \gamma} e^{\frac{2}{\gamma} U_{s}}}-\lambda \frac{1+\Phi_{s}}{\Phi_{s}}\right] d s
$$

for $t \geq 0$. In particular, the process $(U, \Phi)$ solving

$$
\begin{aligned}
& d U_{t}=\left[\frac{\beta}{\Phi_{t}^{2 / \gamma} e^{\frac{2}{\gamma} U_{t}}}-\lambda \frac{1+\Phi_{t}}{\Phi_{t}}\right] d t, \\
& d \Phi_{t}=\lambda\left(1+\Phi_{t}\right) d t+\gamma \frac{\Phi_{t}^{1-1 / \gamma}}{e^{\frac{1}{\gamma} U_{t}}} d B_{t}
\end{aligned}
$$

is a (time-homogeneous) strong Markov process under $\mathrm{P}_{u, \varphi}^{\infty}$ with $\mathrm{P}_{u, \varphi}^{\infty}\left(\left(U_{0}, \Phi_{0}\right)=\right.$ $(u, \varphi))=1$ for $(u, \varphi) \in \mathbb{R} \times[0, \infty)$.

PROOF. Inserting (3.2) and (3.8) into (6.1) and (6.2), it is easily seen that (6.13) holds. Rewriting (6.3)-(6.6) in terms of $F$ and $K$ given in (6.13), we obtain (6.14)-(6.17) as claimed and the proof is complete.

3. Note from (6.8) above that

$$
L_{t}=e^{F\left(X_{t}\right)-F\left(X_{0}\right)+\int_{0}^{t} K\left(X_{s}\right) d s}
$$

for $t \geq 0$ with $F$ and $K$ given in (6.1) and (6.2). When specialised to the case of Bessel processes with (6.13) being satisfied this relation reads

$$
L_{t}=\left(\frac{X_{t}}{X_{0}}\right)^{\gamma} e^{-\beta A_{t}},
$$

where $A=\left(A_{t}\right)_{t \geq 0}$ is an additive functional defined by

$$
A_{t}=\int_{0}^{t} \frac{d s}{X_{s}^{2}}
$$

for $t \geq 0$. The process $A$ is known to play an important role in time changing $X$ as discussed in the next section. Recalling the closed form expression (3.12) for $\Phi_{t}$ in terms of $\left(L_{s}\right)_{0 \leq s \leq t}$ we see from (6.18)-(6.20) how the observed sample path $\left(X_{s}\right)_{0 \leq s \leq t}$ builds $\Phi_{t}$, and thus yields an optimal stopping strategy as discussed in the first paragraph above. 
4. The result of Proposition 4 and Corollary 5 shows that the optimal stopping problem (4.19) for the strong Markov process $(\Phi, X)$ solving (4.7) + (4.8) [or $(4.20)+(4.21)$ in the case of Bessel processes] can be considered as an optimal stopping problem for the strong Markov process $(U, \Phi)$ solving $(6.5)+(6.6)$ [or $(6.16)+(6.17)$ in the case of Bessel processes], respectively. The two optimal stopping problems are equivalent and we will make use of this equivalence in the sequel. The advantage of the latter problem is that the process $U$ is of bounded variation. The advantage of the former problem in the case of Bessel processes is that $X$ can be time changed into a geometric Brownian motion for which explicit calculations in terms of the initial point are possible. This time-change technique will be discussed in the next section.

5. The arguments used in the proof of Proposition 4 for the passage from $(\Phi, X)$ to $(U, \Phi)$ are probabilistic. We now show using purely analytic arguments that this passage is equivalent to the standard reduction of the parabolic equation

$$
\begin{aligned}
\mathbb{L}_{\Phi, X}= & \lambda(1+\varphi) \partial_{\varphi}+\mu_{0}(x) \partial_{x}+\varphi \rho(x) \sigma(x) \partial_{\varphi x} \\
& +\frac{1}{2} \varphi^{2} \rho^{2}(x) \partial_{\varphi \varphi}+\frac{1}{2} \sigma^{2}(x) \partial_{x x}
\end{aligned}
$$

to its canonical form

$$
\begin{aligned}
\mathbb{L}_{U, \Phi}= & {\left[K\left(F^{-1}(\log \varphi+u)\right)-\lambda \frac{1+\varphi}{\varphi}\right] \partial_{u} } \\
& +\lambda(1+\varphi) \partial_{\varphi}+\frac{1}{2} \rho^{2}\left(F^{-1}(\log \varphi+u)\right) \varphi^{2} \partial_{\varphi \varphi},
\end{aligned}
$$

where $\mathbb{L}_{\Phi, X}$ and $\mathbb{L}_{U, \Phi}$ denote the infinitesimal generators of $(\Phi, X)$ to $(U, \Phi)$, respectively. For this, let us name the coefficients in (6.21) by setting

$$
\begin{aligned}
& a(\varphi, x)=\frac{1}{2} \varphi^{2} \rho^{2}(x), \quad 2 b(\varphi, x)=\varphi \rho(x) \sigma(x) \quad \text { and } \\
& c(\varphi, x)=\frac{1}{2} \sigma^{2}(x)
\end{aligned}
$$

for $(\varphi, x) \in[0, \infty) \times(0, \infty)$ given and fixed. Then

$$
b^{2}(\varphi, x)-a(\varphi, x) c(\varphi, x)=\frac{1}{4} \varphi^{2} \rho^{2}(x) \sigma^{2}(x)-\frac{1}{4} \varphi^{2} \rho^{2}(x) \sigma^{2}(x)=0
$$

showing that the equation for $\mathbb{L}_{\Phi, X}$ in (6.21) is parabolic. Moreover, its unique family of characteristic curves (obtained by letting $u$ to be constant below) is given by

$$
\begin{aligned}
\frac{d x}{d \varphi} & =\frac{b(\varphi, x)}{a(\varphi, x)}=\frac{\sigma(x)}{\varphi \rho(x)} \quad \Leftrightarrow \quad \frac{\rho(x)}{\sigma(x)} d x=\frac{d \varphi}{\varphi} \\
\Leftrightarrow F^{\prime}(x) d x & =\frac{d \varphi}{\varphi} \quad \Leftrightarrow \quad F(x)=\log \varphi+u,
\end{aligned}
$$


where we note that the final identity coincides with the identity (6.3) above. Setting $\xi(\varphi, x)=u=F(x)-\log \varphi$ and $\eta(\varphi, x)=\varphi$, we see that the Jacobian $J=\partial(\xi, \eta) / \partial(\varphi, x)=\xi_{\varphi} \eta_{x}-\xi_{x} \eta_{\varphi}=-F^{\prime}(x)=-\rho(x) / \sigma(x) \notin\{0, \infty\}$ as needed (for the inverse function theorem). It follows that the coefficients $A(u, \varphi)$ and $B(u, \varphi)$ associated with $\partial_{u u}$ and $\partial_{u \varphi}$ in the resulting equation (6.22) are zero (as is already visible due to the result of Proposition 4). This establishes a full equivalence between the probabilistic and analytic approach as claimed. The canonical equation (6.22) is easily specialised to the setting of Bessel processes using (6.13) above [or this can be also read off more directly from $(6.16)+(6.17)$ above] yielding

$$
\mathbb{L}_{U, \Phi}=\left[\frac{\beta}{\varphi^{2 / \gamma} e^{\frac{2}{\gamma} u}}-\lambda \frac{1+\varphi}{\varphi}\right] \partial_{u}+\lambda(1+\varphi) \partial_{\varphi}+\frac{\gamma^{2}}{2} \frac{\varphi^{2-2 / \gamma}}{e^{\frac{2}{\gamma} u}} \partial_{\varphi \varphi}
$$

for $(u, \varphi) \in \mathbb{R} \times[0, \infty)$.

6. Note that the process $U$ as the first component of the strong Markov process $(U, \Phi)$ can be either increasing or decreasing depending on the position of $(U, \Phi)$ in the state space $\mathbb{R} \times[0, \infty)$ at which the integrand in (6.4) is positive or negative, respectively. Specialising to the case of Bessel processes when $U$ is given by (6.15), it is easily verified that the turning curve $z$ is given by the expression

$$
z(\varphi)=\frac{\gamma}{2} \log \left(\frac{\beta}{\lambda} \frac{\varphi^{1-2 / \gamma}}{1+\varphi}\right)
$$

for $\varphi \in(0, \infty)$. It means that the state space $\mathbb{R} \times[0, \infty)$ splits into three disjoint sets $P=\{(u, \varphi) \in \mathbb{R} \times[0, \infty) \mid u<z(\varphi)\}, Z=\{(u, \varphi) \in \mathbb{R} \times[0, \infty) \mid u=z(\varphi)\}$, $N=\{(u, \varphi) \in \mathbb{R} \times[0, \infty) \mid u>z(\varphi)\}$ and when the process $(U, \Phi)$ belongs to $P$ or $N$ its first component $U$ is increasing or decreasing, respectively. This shows that the evolutionary character of the parabolic equation (6.26) is fully exhibited only in the sets $P$ and $N$ alone while $Z$ serves as a crossing set. It is easily seen that $z(\infty)=-\infty$ and $z(0)$ equals $\infty$ or $-\infty$ depending on whether $\gamma<2$ or $\gamma>2$ while $z(0)$ takes a finite value in $\mathbb{R}$ when $\gamma=2$. Moreover, if $\gamma \leq 2$ then it is easily verified that $\varphi \mapsto z(\varphi)$ is decreasing on $(0, \infty)$. This shows that when $\gamma \leq 2$ the process $(U, \Phi)$ "circles" around the turning curve $\varphi \mapsto z(\varphi)$ anticlockwise. On the other hand, if $\gamma>2$ then it is easily verified that $\varphi \mapsto z(\varphi)$ is increasing on $(0,(\gamma / 2)-1)$ and decreasing on $((\gamma / 2)-1, \infty)$. In the former case, we thus have that the process $(U, \Phi)$ "circles" around the turning curve $\varphi \mapsto z(\varphi)$ clockwise and in the latter case we similarly have that the process $(U, \Phi)$ "circles" around the turning curve $\varphi \mapsto z(\varphi)$ anticlockwise as in the remaining cases above. Bearing in mind these facts helps to visualise the motion of $(U, \Phi)$ which in turn proves useful in the treatment of the optimal stopping problem (4.19) below. 
7. Time change. In this section, we return to the optimal stopping problem (4.19) and expose key elements of the time-change technique which will be applied in the following sections. The usefulness of the time change itself is twofold. First, it will enable us to realise the second component of the time-changed process (geometric Brownian motion) as an explicit Markovian functional of the initial point. This simplifies the arguments in the sequel and makes a more penetrating analysis of the optimal stopping problem possible. Second, it will enable us to remove dependence of the diffusion coefficient in the first component of the timechanged process on its second component. This makes it possible to use known comparison theorems for the resulting system of coupled stochastic differential equations and will be particularly useful in relation to regularity questions in the subsequent analysis below:

1. To make the time change note that sample paths of the additive functional $A=\left(A_{t}\right)_{t \geq 0}$ defined by (6.20) are continuous and strictly increasing with $A_{0}=0$ and $A_{t} \uparrow \infty$ as $t \uparrow \infty$ (the latter property is well known for Bessel processes $X$ of dimension $\delta \geq 2$ but will also be verified below). Hence, the same properties hold for its inverse $T=\left(T_{t}\right)_{t \geq 0}$ defined by

$$
T_{t}=A_{t}^{-1}
$$

for $t \geq 0$. Since $A$ is adapted to $\left(\mathcal{F}_{t}^{X}\right)_{t \geq 0}$, it follows that each $T_{t}$ is a stopping time with respect to $\left(\mathcal{F}_{t}^{X}\right)_{t \geq 0}$ so that $T=\left(T_{t}\right)_{t \geq 0}$ defines a time change relative to $\left(\mathcal{F}_{t}^{X}\right)_{t \geq 0}$. The fact that $t \mapsto T_{t}$ is continuous and strictly increasing with $T_{t}<\infty$ for $t \geq 0$ (or equivalently $A_{t} \uparrow \infty$ as $t \uparrow \infty$ ) implies that standard time change transformations are applicable to continuous semimartingales and their stochastic integrals without extra conditions on their sample paths (see, e.g., [16], pages 7-9 and pages 179-181) and they will be used in the sequel without explicit mention. Moreover, since $(\Phi, X)$ is a strong Markov process by the well-known result dating back to [21] (see, e.g., [17], page 175, for a modern exposition) we know that the time-changed process $(\hat{\Phi}, \hat{X})=\left(\left(\hat{\Phi}_{t}, \hat{X}_{t}\right)\right)_{t \geq 0}$ defined by

$$
\left(\hat{\Phi}_{t}, \hat{X}_{t}\right)=\left(\Phi_{T_{t}}, X_{T_{t}}\right)
$$

for $t \geq 0$ is a Markov process under $\mathrm{P}_{\varphi, x}^{\infty}$ for $(\varphi, x) \in[0, \infty) \times(0, \infty)$. It is possible to verify that $(\Phi, X)$ is a Feller process, and hence by the same well-known result we could also conclude that $(\hat{\Phi}, \hat{X})$ is a strong Markov process, however, strong Feller property of $(\Phi, X)$ and $(\hat{\Phi}, \hat{X})$ also follows from the existence and uniqueness of a weak solution (cf. [17], page 170) to the systems of stochastic differential equations for $(\Phi, X)$ and $(\hat{\Phi}, \hat{X})$ derived above and below. Moreover, from (6.20) one can read off that the infinitesimal generator of $(\hat{\Phi}, \hat{X})$ is given by

$$
\mathbb{L}_{\hat{\Phi}, \hat{X}}=x^{2} \mathbb{L}_{\Phi, X},
$$

where $\mathbb{L}_{\Phi, X}$ is the infinitesimal generator of $(\Phi, X)$. Note also that $\sigma=A_{\tau}$ is a stopping time of $(\hat{\Phi}, \hat{X})$ if and only if $\tau=T_{\sigma}$ is a stopping time of $(\Phi, X)$ [where 
we recall that the natural filtration of $(\hat{\Phi}, \hat{X})$ coincides with the time-changed natural filtration of $(\Phi, X)$ given by $\hat{\mathcal{F}}_{t}^{\Phi, X}=\mathcal{F}_{T_{t}}^{\Phi, X}$ for $\left.t \geq 0\right]$. Finally, in addition to (6.20) it is easily seen using (7.1) that

$$
T_{t}=\int_{0}^{t} \hat{X}_{s}^{2} d s
$$

for $t \geq 0$. Below we will make frequent use of this relation also.

2 . Recalling that the process $(\Phi, X)$ solves the system of stochastic differential equations $(4.20)+(4.21)$ and making use of these equations we find that

$$
\begin{aligned}
\hat{\Phi}_{t} & =\Phi_{T_{t}}=\Phi_{0}+\int_{0}^{T_{t}} \lambda\left(1+\Phi_{s}\right) d s+\int_{0}^{T_{t}} \gamma \frac{\Phi_{s}}{X_{s}} d B_{s} \\
& =\hat{\Phi}_{0}+\int_{0}^{t} \lambda\left(1+\hat{\Phi}_{s}\right) \hat{X}_{s}^{2} d s+\int_{0}^{t} \gamma \hat{\Phi}_{s} d \tilde{B}_{s}, \\
\hat{X}_{t} & =X_{T_{t}}=X_{0}+\int_{0}^{T_{t}} \frac{\delta_{0}-1}{2 X_{s}} d s+\int_{0}^{T_{t}} d B_{s} \\
& =\hat{X}_{0}+\int_{0}^{t} \frac{\delta_{0}-1}{2} \hat{X}_{s} d s+\int_{0}^{t} \hat{X}_{s} d \tilde{B}_{s},
\end{aligned}
$$

where the process $\tilde{B}=\left(\tilde{B}_{t}\right)_{t \geq 0}$ is defined by

$$
\tilde{B}_{t}=\int_{0}^{t} \frac{d \hat{B}_{s}}{\hat{X}_{s}}=\int_{0}^{T_{t}} \frac{d B_{s}}{X_{s}}=M_{T_{t}}
$$

upon setting $M_{t}=\int_{0}^{t} d B_{s} / X_{s}$ for $t \geq 0$. Since $M=\left(M_{t}\right)_{t \geq 0}$ is a continuous local martingale with respect to $\left(\mathcal{F}_{t}^{X}\right)_{t \geq 0}$, it follows that $\tilde{B}=\left(\tilde{B}_{t}\right)_{t \geq 0}$ is a continuous local martingale with respect to $\left(\mathcal{F}_{t}^{X}\right)_{t \geq 0}$. Note moreover that $\langle\tilde{B}, \tilde{B}\rangle_{t}=$ $\left\langle M_{T}, M_{T}\right\rangle_{t}=\langle M, M\rangle_{T_{t}}=\int_{0}^{T_{t}} d s / X_{s}^{2}=A_{T_{t}}=t$ for $t \geq 0$. Hence, by Lévy's characterisation theorem (see, e.g., [16], page 150) we can conclude that $\tilde{B}$ is a standard Brownian motion with respect to $\left(\mathcal{F}_{t}^{X}\right)_{t \geq 0}$. It follows therefore that $(7.5)+(7.6)$ can be written as the following stochastic differential equations:

$$
\begin{aligned}
& d \hat{\Phi}_{t}=\lambda\left(1+\hat{\Phi}_{t}\right) \hat{X}_{t}^{2} d t+\gamma \hat{\Phi}_{t} d \tilde{B}_{t}, \\
& d \hat{X}_{t}=\left(\frac{\delta_{0}-1}{2}\right) \hat{X}_{t} d t+\hat{X}_{t} d \tilde{B}_{t}
\end{aligned}
$$

under $\mathrm{P}_{\varphi, x}^{\infty}$ for $(\varphi, x) \in[0, \infty) \times(0, \infty)$. Recalling known sufficient conditions (see, e.g., [17], pages 166-173) we see that the system of stochastic differential equations $(7.8)+(7.9)$ has a unique weak solution, and hence by the well-known result (see, e.g., [17], pages 158-163) we can conclude that $(\hat{\Phi}, \hat{X})$ is a (timehomogeneous) strong Markov process under $\mathrm{P}_{\varphi, x}^{\infty}$ for $(\varphi, x) \in[0, \infty) \times(0, \infty)$. 
From (7.9), we see that $\hat{X}$ defines a geometric Brownian motion, and hence the following explicit representation is satisfied:

$$
\hat{X}_{t}^{x}=x \exp \left(\tilde{B}_{t}+\left(\frac{\delta_{0}}{2}-1\right) t\right)
$$

for $t \geq 0$ and $x \in(0, \infty)$ under $\mathrm{P}^{\infty}$ with respect to which $\tilde{B}$ is a standard Brownian motion. This fact will be useful in the subsequent analysis. From (7.10), one can also easily verify that $\lim _{t \rightarrow \infty} A_{t}$ cannot be finite as stated above. Observe moreover that the diffusion coefficient in (7.8) no longer depends on the process $\hat{X}$ from (7.9). Finally, note that in exactly the same way as in (7.5)-(7.9) above, we find that the time-changed process $\hat{L}=\left(L_{T_{t}}\right)_{t \geq 0}$ of $L$ from (4.9) and (4.10) solves the following stochastic differential equation:

$$
d \hat{L}_{t}=\gamma \hat{L}_{t} d \tilde{B}_{t}
$$

and hence is given explicitly by

$$
\hat{L}_{t}=\exp \left(\gamma \tilde{B}_{t}-\frac{\gamma^{2}}{2} t\right)
$$

for $t \geq 0$. This fact will also be handy in the subsequent analysis.

3. We can now make use of the previous facts and derive a time-changed version of the optimal stopping problem (4.19) above.

Proposition 6. The value function $\hat{V}$ from (4.19) satisfies the identity

$$
\hat{V}(\varphi, x)=\inf _{\sigma} \mathrm{E}_{\varphi, x}^{\infty}\left[\int_{0}^{\sigma} e^{-\lambda \int_{0}^{t} \hat{X}_{s}^{2} d s}\left(\hat{\Phi}_{t}-\frac{\lambda}{c}\right) \hat{X}_{t}^{2} d t\right]
$$

for $(\varphi, x) \in[0, \infty) \times(0, \infty)$ where the infimum is taken over all stopping times $\sigma$ of $(\hat{\Phi}, \hat{X})$.

PROOF. Recall that $\tau=T_{\sigma}$ is a stopping time of $(\Phi, X)$ if and only if $\sigma=A_{\tau}$ is a stopping time of $(\hat{\Phi}, \hat{X})$. Thus, if either $\tau$ or $\sigma$ is given we can form $\sigma$ or $\tau$, respectively, and using (7.4) note that

$$
\begin{aligned}
\mathrm{E}_{\varphi, x}^{\infty}\left[\int_{0}^{\tau} e^{-\lambda t}\left(\Phi_{t}-\frac{\lambda}{c}\right) d t\right] & =\mathrm{E}_{\varphi, x}^{\infty}\left[\int_{0}^{T_{\sigma}} e^{-\lambda t}\left(\Phi_{t}-\frac{\lambda}{c}\right) d t\right] \\
& =\mathrm{E}_{\varphi, x}^{\infty}\left[\int_{0}^{\sigma} e^{-\lambda T_{t}}\left(\Phi_{T_{t}}-\frac{\lambda}{c}\right) d T_{t}\right] \\
& =\mathrm{E}_{\varphi, x}^{\infty}\left[\int_{0}^{\sigma} e^{-\lambda \int_{0}^{t} \hat{X}_{s}^{2} d s}\left(\hat{\Phi}_{t}-\frac{\lambda}{c}\right) \hat{X}_{t}^{2} d t\right] .
\end{aligned}
$$

Taking the infimum over all $\tau$ and/or $\sigma$ on both sides of (7.14) as above we see that (7.13) holds as claimed and the proof is complete. 
It follows from Proposition 6 that the optimal stopping problem (4.19) is equivalent to the optimal stopping problem defined on the right-hand side of (7.13) for the strong Markov process $(\hat{\Phi}, \hat{X})$ solving the system of stochastic differential equations $(7.8)+(7.9)$ under $\mathrm{P}_{\varphi, x}$ for $(\varphi, x) \in[0, \infty) \times(0, \infty)$. This equivalence will be exploited in the next section when deriving basic properties of the optimal stopping boundary.

8. Monotonicity of the optimal stopping boundary. In this section, we establish the existence of an optimal stopping time in (4.19) and derive basic properties of the optimal stopping boundary.

1. Recalling that the Lagrange formulated problem (4.19) and the Mayer formulated problem (5.13) are equivalent, we may conclude that the (candidate) continuation and stopping sets in these problems need to be defined as follows:

$$
\begin{aligned}
C & =\{(\varphi, x) \in[0, \infty) \times(0, \infty) \mid \hat{V}(\varphi, x)<0\} \\
& =\{(\varphi, x) \in[0, \infty) \times(0, \infty) \mid \check{V}(\varphi, x)<M(\varphi, x)\}, \\
D & =\{(\varphi, x) \in[0, \infty) \times(0, \infty) \mid \hat{V}(\varphi, x)=0\} \\
& =\{(\varphi, x) \in[0, \infty) \times(0, \infty) \mid \check{V}(\varphi, x)=M(\varphi, x)\},
\end{aligned}
$$

respectively. Using the fact that $L$ from (3.10) is a martingale it is easily verified that the right-hand side of (3.12) defines a Markovian functional of the initial point. Time changing (5.13) by (7.1) and recalling that (7.10) also defines a Markovian functional of the initial point, we see that the expectation in (5.13) defines a continuous function of the initial point $(\varphi, x)$ for every (bounded) stopping time $\tau$ given and fixed. Taking the infimum over all (bounded) stopping times $\tau$, we can thus conclude that the value function $\check{V}$ is upper semicontinuous. From (5.12), we see that the loss function $M$ is continuous, and hence lower semicontinuous as well. It follows therefore by [15], Corollary 2.9, that the first entry time of the process $(\Phi, X)$ into the closed set $D$ defined by

$$
\tau_{D}=\inf \left\{t \geq 0 \mid\left(\Phi_{t}, X_{t}\right) \in D\right\}
$$

is optimal in (4.19) and (5.13) whenever $\mathrm{P}_{\varphi, x}\left(\tau_{D}<\infty\right)=1$ for all $(\varphi, x) \in$ $[0, \infty) \times(0, \infty)$. In the sequel, we will establish this and other properties of $\tau_{D}$ by analysing the boundary of $D$.

2. Since the integrand in (4.19) is strictly negative for $\varphi<\lambda / c$, it is clear that this region of the state space is contained in $C$ [otherwise the first exit times of $(\Phi, X)$ from sufficiently small neighbourhoods would violate stopping at once]. To expand on this further, let us formally define the (least) boundary between $C$ and $D$ by setting

$$
b(x)=\inf \{\varphi \geq 0 \mid(\varphi, x) \in D\}
$$


for every $x>0$ given and fixed. Clearly, $b(x) \geq \lambda / c$ and the infimum in (8.4) is attained for every $x>0$ since $D$ is closed. From (3.12), we see that $\varphi \mapsto \Phi^{\varphi, x}$ is increasing on $[0, \infty)$ for every $x>0$ given and fixed (superscripts throughout indicate the initial points of Markov processes), and hence from the structure of (4.19) it is evident that

$$
\varphi \mapsto \hat{V}(\varphi, x) \text { is increasing on }[0, \infty)
$$

for every $x>0$ given and fixed. This shows that if $\left(\varphi_{1}, x\right) \in D$ and $\varphi_{2} \geq \varphi_{1}$ then $\left(\varphi_{2}, x\right) \in D$ because $0=\hat{V}\left(\varphi_{1}, x\right) \leq \hat{V}\left(\varphi_{2}, x\right) \leq 0$ so that $\hat{V}\left(\varphi_{2}, x\right)=0$ as well implying the claim. From this fact, it follows that $b$ from (8.4) defines the (entire) boundary between $C$ and $D$. The key property of $b$ will be verified shortly below as a consequence of the following fact.

LEMMA 7. The following inequality holds:

$$
\hat{V}(\varphi, y) \geq \frac{y^{2}}{x^{2}} \hat{V}(\varphi, x)
$$

for all $\varphi \geq 0$ and all $y \geq x>0$.

ProOF. By the time change (7.1), we know using the result of Proposition 6 that the value function $\hat{V}$ from (4.19) is given by the right-hand side of (7.13). To analyse the latter, we will first reduce the complexity arising from a time-changed equation (3.12) regarding the initial point and pass to another stochastic differential equation instead.

1. Set $\Psi_{t}=e^{-\lambda t} \Phi_{t}$ for $t \geq 0$ and note that integration by parts using (4.12) yields

$$
d \Psi_{t}=\lambda e^{-\lambda t} d t+\gamma \frac{\Psi_{t}}{X_{t}} d B_{t}
$$

with $\Psi_{0}=\Phi_{0}$. Time changing $\Psi$ by setting $\hat{\Psi}_{t}=\Psi_{T_{t}}$ where $T_{t}$ is given by (7.1) for $t \geq 0$ we find in exactly the same way as in (7.8) that

$$
d \hat{\Psi}_{t}=\lambda e^{-\lambda T_{t}} \hat{X}_{t}^{2} d t+\gamma \hat{\Psi}_{t} d \tilde{B}_{t},
$$

where by (7.4) we also have

$$
d T_{t}=\hat{X}_{t}^{2} d t
$$

as well as by (7.9) we know that

$$
d \hat{X}_{t}=\left(\frac{\delta_{0}-1}{2}\right) \hat{X}_{t} d t+\hat{X}_{t} d \tilde{B}_{t}
$$

with $\left(\hat{\Psi}_{0}, T_{0}, \hat{X}_{0}\right)=(\varphi, 0, x)$ under $\mathrm{P}_{\varphi, x}^{\infty}$ for $(\varphi, x) \in[0, \infty) \times(0, \infty)$. This shows that the process $(\hat{\Psi}, T, \hat{X})$ solves the system of stochastic differential equations 
$(8.8)+(8.9)+(8.10)$ where the diffusion coefficients in (8.8) and (8.10) depend only on $\hat{\Psi}$ and $\hat{X}$, respectively [the analogous fact is also true for (8.9) above]. It follows therefore by the comparison result for solutions to multidimensional stochastic differential equations given in [7], Theorem 1, that

$$
\mathrm{P}^{\infty}\left(\hat{\Psi}_{t}^{\varphi, x} \leq \hat{\Psi}_{t}^{\varphi, y} \text { for all } t \geq 0\right)=1
$$

for $\varphi \in[0, \infty)$ and $x \leq y$ in $(0, \infty)$ where the superscripts $\varphi, x$ indicate dependence on the initial points $\hat{\Psi}_{0}=\varphi, \hat{X}_{0}=x$ and dependence on the initial point $T_{0}=0$ is ignored (as it plays no role in the argument below).

2. Let $\varphi \in[0, \infty)$ and $x \leq y$ in $(0, \infty)$ be given and fixed. For $\varepsilon>0$ given and fixed, we can choose a stopping time $\tau_{\varepsilon}=\tau_{\varepsilon}(\varphi, y)$ such that

$$
\hat{V}(\varphi, y)+\varepsilon \geq \mathrm{E}_{\varphi, y}^{\infty}\left[\int_{0}^{\tau_{\varepsilon}} e^{-\lambda t}\left(\Phi_{t}-\frac{\lambda}{c}\right) d t\right] .
$$

To indicate dependence of $A_{t}$ from (6.20) on the initial point $x$ of $X^{x}$ through $\left(X_{s}^{x}\right)_{0 \leq s \leq t}$ in the integral, we will write $A_{t}^{(x)}$, and similarly for its inverse $T_{t}$ from (7.1) we will write $T_{t}^{(x)}$ in the sequel. Note from (7.4) and (7.10) that $T_{t}^{(x)}=$ $x^{2} T_{t}^{(1)}$ for $x>0$. Similarly, from (7.10) we see that $\hat{X}_{t}^{x}=x \hat{X}_{t}^{1}$ for $x>0$. We will use these facts freely below with no further mention.

Setting $\sigma_{\varepsilon}=A_{\tau_{\varepsilon}}^{(y)}$ we find similarly to (7.14) above using the same notation/arguments that the following facts are satisfied:

$$
\begin{aligned}
\mathrm{E}_{\varphi, y}^{\infty} & {\left[\int_{0}^{\tau_{\varepsilon}} e^{-\lambda t}\left(\Phi_{t}-\frac{\lambda}{c}\right) d t\right] } \\
& =\mathrm{E}^{\infty}\left[\int_{0}^{\tau_{\varepsilon}} e^{-\lambda t}\left(\Phi_{t}^{\varphi, y}-\frac{\lambda}{c}\right) d t\right] \\
& =\mathrm{E}^{\infty}\left[\int_{0}^{T_{\sigma \varepsilon}^{(y)}} \Psi_{t}^{\varphi, y} d t-\frac{\lambda}{c} \int_{0}^{T_{\sigma \varepsilon}^{(y)}} e^{-\lambda t} d t\right] \\
& =\mathrm{E}^{\infty}\left[\int_{0}^{\sigma_{\varepsilon}} \hat{\Psi}_{t}^{\varphi, y}\left(\hat{X}_{t}^{y}\right)^{2} d t-\frac{\lambda}{c} \int_{0}^{\sigma_{\varepsilon}} e^{-\lambda T_{t}^{(y)}}\left(\hat{X}_{t}^{y}\right)^{2} d t\right] \\
& \geq \mathrm{E}^{\infty}\left[\int_{0}^{\sigma_{\varepsilon}} \hat{\Psi}_{t}^{\varphi, x}\left(\hat{X}_{t}^{y}\right)^{2} d t-\frac{\lambda}{c} \int_{0}^{\sigma_{\varepsilon}} e^{-\lambda T_{t}^{(y)}}\left(\hat{X}_{t}^{y}\right)^{2} d t\right] \\
& \geq \mathrm{E}^{\infty}\left[\int_{0}^{\sigma_{\varepsilon}} \hat{\Psi}_{t}^{\varphi, x}\left(y \hat{X}_{t}^{1}\right)^{2} d t-\frac{\lambda}{c} \int_{0}^{\sigma_{\varepsilon}} e^{-\lambda x^{2} T_{t}^{(1)}}\left(y \hat{X}_{t}^{1}\right)^{2} d t\right] \\
& =\frac{y^{2}}{x^{2}} \mathrm{E}^{\infty}\left[\int_{0}^{\sigma_{\varepsilon}} \hat{\Psi}_{t}^{\varphi, x}\left(\hat{X}_{t}^{x}\right)^{2} d t-\frac{\lambda}{c} \int_{0}^{\sigma_{\varepsilon}} e^{-\lambda T_{t}^{(x)}}\left(\hat{X}_{t}^{x}\right)^{2} d t\right] \\
& \geq \frac{y^{2}}{x^{2}} \inf _{\sigma} \mathrm{E}^{\infty}\left[\int_{0}^{\sigma} \hat{\Psi}_{t}^{\varphi, x}\left(\hat{X}_{t}^{x}\right)^{2} d t-\frac{\lambda}{c} \int_{0}^{\sigma} e^{-\lambda T_{t}^{(x)}}\left(\hat{X}_{t}^{x}\right)^{2} d t\right]
\end{aligned}
$$




$$
\begin{aligned}
& =\frac{y^{2}}{x^{2}} \inf _{\sigma} \mathrm{E}_{\varphi, x}^{\infty}\left[\int_{0}^{\sigma} \hat{\Psi}_{t}\left(\hat{X}_{t}\right)^{2} d t-\frac{\lambda}{c} \int_{0}^{\sigma} e^{-\lambda T_{t}}\left(\hat{X}_{t}\right)^{2} d t\right] \\
& =\frac{y^{2}}{x^{2}} \inf _{\sigma} \mathrm{E}_{\varphi, x}^{\infty}\left[\int_{0}^{T_{\sigma}} \Psi_{t} d t-\frac{\lambda}{c} \int_{0}^{T_{\sigma}} e^{-\lambda t} d t\right] \\
& =\frac{y^{2}}{x^{2}} \inf _{\tau} \mathrm{E}_{\varphi, x}^{\infty}\left[\int_{0}^{\tau} e^{-\lambda t}\left(\Phi_{t}-\frac{\lambda}{c}\right) d t\right]=\frac{y^{2}}{x^{2}} \hat{V}(\varphi, x),
\end{aligned}
$$

where in the first inequality we use (8.11) above. Combining (8.12) and (8.13), and letting $\varepsilon \rightarrow 0$, we see that (8.6) holds as claimed and the proof is complete.

We can now derive the following important consequence of Lemma 7 about the boundary $b$ between the sets $C$ and $D$.

COROLlaRY 8. The mapping $x \mapsto b(x)$ is decreasing on $(0, \infty)$.

Proof. If $(\varphi, x) \in D$ and $y \geq x$, then by (8.6) we see that

$$
0 \geq \hat{V}(\varphi, y) \geq \frac{y^{2}}{x^{2}} \hat{V}(\varphi, x)=0
$$

so that $\hat{V}(\varphi, y)=0$, and hence $(\varphi, y) \in D$. This shows using (8.4) above that $b$ is decreasing on $(0, \infty)$ as claimed and the proof is complete.

3. It is evident from (7.8) and (7.9) using simple comparison arguments (when $\delta_{0}=2$ as well) that the process $(\Phi, X)$ hits every quadrant $\left\{\left(\varphi^{\prime}, x^{\prime}\right) \mid \varphi^{\prime} \geq\right.$ $\varphi_{1}$ and $\left.x^{\prime} \geq x_{1}\right\}$ with $\mathrm{P}_{\varphi, x}$-probability one whenever $0 \leq \varphi<\varphi_{1}$ and $0<x<x_{1}$ are given and fixed. It follows therefore by the result of Corollary 8 that $\tau_{D}<\infty$ with $\mathrm{P}_{\varphi, x}$-probability one whenever there exists $x_{1}>0$ such that $b\left(x_{1}\right)<\infty$ which in turn is equivalent to $D$ being nonempty. To rule out the possibility that $D$ is empty, note that using (8.7) this would imply that

$$
\begin{aligned}
\hat{V}(\varphi, x) & =\mathrm{E}_{\varphi, x}^{\infty}\left[\int_{0}^{\infty} e^{-\lambda t}\left(\Phi_{t}-\frac{\lambda}{c}\right) d t\right] \\
& =\int_{0}^{\infty}\left[(1+\varphi)-(1+\lambda / c) e^{-\lambda t}\right] d t=\infty
\end{aligned}
$$

which is a contradiction (with the fact that $\hat{V}$ is nonpositive). We can therefore conclude that $\mathrm{P}_{\varphi, x}\left(\tau_{D}<\infty\right)=1$ for all $(\varphi, x) \in[0, \infty) \times(0, \infty)$, and hence $\tau_{D}$ is optimal in (4.19) as stated following (8.3) above.

4. Despite being finite at least one point, and hence at all larger points too, the boundary $b$ could in principle take infinite values as well. We next show that this is not the case.

PROPOSITION 9. We have $b(x)<\infty$ for all $x>0$. 
Proof. Suppose that there exists $x_{1}>0$ such that $b\left(x_{1}\right)=\infty$. Then by the result of Corollary 8 we know that $b(x)=\infty$ for all $x \in\left(0, x_{1}\right]$. Fix any $x \in\left(0, x_{1}\right)$ (e.g., take $x=x_{1} / 2$ or similar) and consider the stopping time $\tau_{1}=\inf \left\{t \geq 0 \mid X_{t} \geq\right.$ $\left.x_{1}\right\}$ under the measure $\mathrm{P}_{\varphi, x}$ where $\varphi>0$ is given and fixed. Then clearly $\tau_{D} \geq \tau_{1}$ so that

$$
\begin{aligned}
\hat{V}(\varphi, x) & =\mathrm{E}_{\varphi, x}^{\infty}\left[\int_{0}^{\tau_{D}} e^{-\lambda t}\left(\Phi_{t}-\frac{\lambda}{c}\right) d t\right] \\
& \geq \mathrm{E}_{\varphi, x}^{\infty}\left[\int_{0}^{\tau_{1}} e^{-\lambda t} \Phi_{t} d t\right]+\frac{1}{c}\left[\mathrm{E}_{\varphi, x}^{\infty}\left(e^{-\lambda \tau_{D}}\right)-1\right] \\
& \geq \mathrm{E}^{\infty}\left[\int_{0}^{\tau_{1}^{(x)}} L_{t}^{(x)}\left(\varphi+\lambda \int_{0}^{t} \frac{d s}{e^{\lambda s} L_{s}^{(x)}}\right) d t\right]-\frac{1}{c} \\
& \geq \varphi \mathrm{E}^{\infty}\left[\int_{0}^{\tau_{1}^{(x)}} L_{t}^{(x)} d t\right]-\frac{1}{c},
\end{aligned}
$$

where in the second inequality we make use of (3.12) above. Using that the final integral in (8.16) is strictly positive and taking $\varphi>0$ large enough we can make the final expression in (8.16) strictly positive so that $\hat{V}(\varphi, x)>0$ which is a contradiction. It follows therefore that there is no $x_{1}>0$ such that $b\left(x_{1}\right)=\infty$ as claimed and the proof is complete.

5. Recalling from Corollary 8 and Proposition 9 that $b$ is decreasing and finite valued, we know that the limit of $b(x)$ exists and is finite as $x \rightarrow \infty$. We now identify this limit as the constant appearing in the integrand of (4.19) above.

\section{PROPOSITION 10. The following relation holds:}

$$
\lim _{x \rightarrow \infty} b(x)=\frac{\lambda}{c}
$$

Proof. Since the set $\{(\varphi, x) \in[0, \infty) \times(0, \infty) \mid \varphi<\lambda / c\}$ is contained in $C$, we see that $\ell_{1}:=\lim _{x \rightarrow \infty} b(x) \geq \lambda / c$. Suppose that $\ell_{1}>\lambda / c$. Choose $\delta>0$ small enough so that $\ell_{0}:=(\lambda / c)+\delta<\ell_{1}$ and let $\varphi \in\left(\ell_{0}, \ell_{1}\right)$ be given and fixed (take the mid point for instance). Consider $\tau_{\ell_{0}, \ell_{1}}=\inf \left\{t \geq 0 \mid \Phi_{t} \notin\left(\ell_{0}, \ell_{1}\right)\right\}$ and note from (4.20) that $\Phi^{\varphi, x} \rightarrow \Phi^{\varphi, \infty}$ uniformly on finite time intervals with $\mathrm{P}^{\infty}$-probability one as $x \rightarrow \infty$ where $\Phi^{\varphi, \infty}=\left(\Phi_{t}^{\varphi, \infty}\right)_{t \geq 0}$ is a deterministic process with a strictly positive drift solving (4.20) without the stochastic differential on the right-hand side. A quick way to establish this convergence is to note from (4.10) (using the Burkholder-Davis-Gundy inequality (see, e.g., [16], page 161) applied to the stochastic integral appearing there) that $L^{(x)} \rightarrow 1$ uniformly on finite time intervals with $\mathrm{P}^{\infty}$-probability one as $x \rightarrow \infty$ and then make use of this fact in (3.12) above. Setting $\tau_{\ell_{1}}=\inf \left\{t \geq 0 \mid \Phi_{t} \geq \ell_{1}\right\}$ it follows therefore that $\tau_{\ell_{0}, \ell_{1}}^{\varphi, x} \rightarrow \tau_{\ell_{1}}^{\varphi, \infty}$ as 
well as $\tau_{\ell_{0}, \ell_{1}}^{\varphi, x}-\tau_{D} \rightarrow 0$ with $\mathrm{P}^{\infty}$-probability one as $x \rightarrow \infty$. Using these facts with $\tau_{\ell_{0}, \ell_{1}} \leq \tau_{D}$, we find that

$$
\begin{aligned}
\hat{V}(\varphi, x)= & \mathrm{E}_{\varphi, x}^{\infty}\left[\int_{0}^{\tau_{D}} e^{-\lambda t}\left(\Phi_{t}-\frac{\lambda}{c}\right) d t\right] \\
\geq & \mathrm{E}_{\varphi, x}^{\infty}\left[\int_{0}^{\tau_{\ell_{0}, \ell_{1}}} e^{-\lambda t} \Phi_{t} d t\right]+\frac{1}{c}\left[\mathrm{E}_{\varphi, x}^{\infty}\left(e^{-\lambda \tau_{D}}\right)-1\right] \\
\geq & \ell_{0} \mathrm{E}_{\varphi, x}^{\infty}\left[\int_{0}^{\tau_{\ell_{0}, \ell_{1}}} e^{-\lambda t} d t\right]+\frac{1}{c}\left[\mathrm{E}_{\varphi, x}^{\infty}\left(e^{-\lambda \tau_{D}}\right)-1\right] \\
= & \frac{1}{c}\left[1-\mathrm{E}_{\varphi, x}^{\infty}\left(e^{-\lambda \tau_{\ell_{0}, \ell_{1}}}\right)\right]+\frac{1}{c}\left[\mathrm{E}_{\varphi, x}^{\infty}\left(e^{-\lambda \tau_{D}}\right)-1\right] \\
& +\frac{\delta}{\lambda}\left[1-\mathrm{E}_{\varphi, x}^{\infty}\left(e^{-\lambda \tau_{\ell_{0}, \ell_{1}}}\right)\right] \\
= & \frac{1}{c}\left[\mathrm{E}_{\varphi, x}^{\infty}\left(e^{-\lambda \tau_{D}}\right)-\mathrm{E}_{\varphi, x}^{\infty}\left(e^{-\lambda \tau_{\ell_{0}, \ell_{1}}}\right)\right]+\frac{\delta}{\lambda}\left[1-\mathrm{E}_{\varphi, x}^{\infty}\left(e^{-\lambda \tau_{\ell_{0}, \ell_{1}}}\right)\right] \\
= & \frac{1}{c} \mathrm{E}^{\infty}\left(e^{-\lambda \tau_{D}^{\varphi, x}}-e^{-\lambda \tau_{\ell_{0}, \ell_{1}}^{\varphi, x}}\right)+\frac{\delta}{\lambda}\left[1-\mathrm{E}^{\infty}\left(e^{-\lambda \tau_{\ell_{0}, \ell_{1}}^{\varphi, x}}\right)\right] \\
\rightarrow & \frac{\delta}{\lambda}\left[1-\mathrm{E}^{\infty}\left(e^{-\lambda \tau_{\ell_{1}}^{\varphi, \infty}}\right)\right]>0
\end{aligned}
$$

as $x \rightarrow \infty$ where the final strict inequality follows from the fact that $\Phi^{\varphi, \infty}$ is a continuous deterministic motion to the right starting at $\varphi$ which is strictly smaller than $\ell_{1}$ so that $\tau_{\ell_{1}}^{\varphi, \infty}$ is strictly positive. From (8.18), we see that for $x>0$ large enough we would have that $\hat{V}(\varphi, x)>0$ which is a contradiction (with the fact that $\hat{V}$ is nonpositive). We can therefore conclude that $\ell_{1}$ must be equal to $\lambda / c$ as claimed and the proof is complete.

6. For the reasons recalled prior to Proposition 10, we know that the limit of $b(x)$ exists as $x \downarrow 0$. We now show that this limit is not finite. Note that this also implies that $C$ and $D$ are not trivial (i.e., $b$ is not identically equal to $\lambda / c$ ).

PROPOSITION 11. The following relation holds:

$$
\lim _{x \downarrow 0} b(x)=\infty
$$

PROOF. Dividing both sides of (7.13) by $x^{2}$ upon recalling (7.10) and taking the limsup as $x \downarrow 0$, we see that the question reduces to establish that it is not optimal to stop at once in the optimal stopping problem

$$
\inf _{\sigma} \mathrm{E}^{\infty}\left[\int_{0}^{\sigma}\left(\hat{\Phi}_{t}^{\varphi, 0}-\frac{\lambda}{c}\right)\left(\hat{X}_{t}^{1}\right)^{2} d t\right]
$$


where the infimum is taken over all stopping times $\sigma$ of $\left(\hat{\Phi}^{\varphi, 0}, \hat{X}^{1}\right)$ and $\Phi^{\varphi, 0}$ solves (7.8) with no drift term so that $\hat{\Phi}_{t}^{\varphi, 0}=\varphi \exp \left(\gamma \tilde{B}_{t}-\left(\gamma^{2} / 2\right) t\right)$ for $\varphi>0$ and $t \geq 0$. Hence, we see that $\hat{\Phi}_{t}^{\varphi, 0} \rightarrow 0$ as $t \rightarrow 0$ while from (7.10) we infer that $\lim \sup _{t \rightarrow \infty} \hat{X}_{t}^{1}=\infty$ when $\delta_{0}=2$ and $\hat{X}_{t}^{1} \rightarrow \infty$ as $t \rightarrow \infty$ when $\delta_{0}>2$ all with $\mathrm{P}^{\infty}$-probability one. Combining these facts and defining $\sigma_{-n}:=\{s \geq$ $\left.0 \mid \int_{0}^{s}\left(\hat{\Phi}_{t}^{\varphi, 0}-\lambda / c\right)\left(\hat{X}_{t}^{1}\right)^{2} d t=-n\right\}$, we see that $\sigma_{-n}<\infty$ with $\mathrm{P}^{\infty}$-probability one for every $n \geq 1$. This shows that the infimum in (8.20) equals $-\infty$ so that it is not optimal to stop at once as needed. Combining this fact with the initial limsup equality obtained from (7.13) as $x \downarrow 0$ and the fact that $\hat{\Phi}^{\varphi, x} \rightarrow \hat{\Phi}^{\varphi, 0}$ uniformly on finite time intervals with $\mathrm{P}^{\infty}$-probability one as $x \downarrow 0$, we see that for every $\varphi>0$ as large as desired, there exists $x>0$ small enough such that $\hat{V}(\varphi, x)<0$. This shows that the limit in (8.19) cannot be finite as claimed and the proof is complete.

9. Continuity of the value function. In this section, we prove that the value function $\hat{V}$ from (4.19) is continuous. A key argument in the proof is due to the fact that

$$
\varphi \mapsto \sigma_{D}^{\varphi, x} \quad \text { and } \quad x \mapsto \sigma_{D}^{\varphi, x} \text { are decreasing }
$$

on $[0, \infty)$ and $(0, \infty)$, respectively, where we let

$$
\sigma_{D}^{\varphi, x}=\inf \left\{t \geq 0 \mid\left(\hat{\Phi}_{t}^{\varphi, x}, \hat{X}_{t}^{x}\right) \in D\right\}
$$

denote the optimal stopping time for $\hat{V}(\varphi, x)$ in (7.13) under $\mathrm{P}^{\infty}$ with $(\varphi, x) \in$ $[0, \infty) \times(0, \infty)$ given and fixed. Indeed, noting that the diffusion coefficients in (7.8) and (7.9) depend only on $\hat{\Phi}$ and $\hat{X}$, respectively, we can conclude by the comparison result for solutions to multidimensional stochastic differential equations given in [7], Theorem 1, that

$$
\mathrm{P}^{\infty}\left(\hat{\Phi}_{t}^{\varphi_{1}, x_{1}} \leq \hat{\Phi}_{t}^{\varphi_{2}, x_{2}} \text { and } \hat{X}_{t}^{x_{1}} \leq \hat{X}_{t}^{x_{2}} \text { for all } t \geq 0\right)=1
$$

for $\varphi_{1} \leq \varphi_{2}$ in $[0, \infty)$ and $x_{1} \leq x_{2}$ in $(0, \infty)$ with $t \geq 0$ [the second inequality in (9.3) being also evident from (7.10) above] so that (9.1) follows immediately from the fact established in Corollary 8 above that $x \mapsto b(x)$ is decreasing on $(0, \infty)$. Equipped with this conclusion, we can now state the main result of this section.

Proposition 12. For the value function $\hat{V}$ from (4.19), we have

$$
(\varphi, x) \mapsto \hat{V}(\varphi, x) \text { is continuous }
$$

on $[0, \infty) \times(0, \infty)$.

Proof. To establish (9.4), it is enough to show that

(9.5) $\varphi \mapsto \hat{V}(\varphi, x)$ is continuous at $\varphi_{0}$ uniformly over $x \in\left[x_{0}-\delta, x_{0}+\delta\right]$,

(9.6) $x \mapsto \hat{V}\left(\varphi_{0}, x\right)$ is continuous at $x_{0}$ 
for every $\left(\varphi_{0}, x_{0}\right) \in[0, \infty) \times(0, \infty)$ given and fixed with some $\delta>0$ sufficiently small.

1. To derive (9.5), fix $\varphi_{1} \leq \varphi_{2}$ in $\left[\varphi_{0}-\delta, \varphi_{0}+\delta\right]$ and $x \in\left[x_{0}-\delta, x_{0}+\delta\right]$ for $\delta>0$ such that $\varphi_{0}-\delta>0$ and $x_{0}-\delta>0$ where $\varphi_{0}>0$ and $x_{0}>0$ are given and fixed (the case $\varphi_{0}=0$ can be treated analogously by replacing $\varphi_{0}-\delta$ with 0 throughout). Let $\tau_{D}^{\varphi_{1}, x}$ denote the optimal stopping time for $\hat{V}\left(\varphi_{1}, x\right)$ in (4.19) under $\mathrm{P}^{\infty}$. By the time change (7.1) and the result of Proposition 6, we know that $\sigma_{D}^{\varphi_{1}, x}=T^{(x)} \circ \tau_{D}^{\varphi_{1}, x}$ is the optimal stopping time for $\hat{V}\left(\varphi_{1}, x\right)$ in (7.13) under $\mathrm{P}^{\infty}$. It follows therefore as in (7.14) using (3.12) and (8.5) that

$$
\begin{aligned}
0 \leq & \hat{V}\left(\varphi_{2}, x\right)-\hat{V}\left(\varphi_{1}, x\right) \\
\leq & \mathrm{E}^{\infty}\left[\int_{0}^{\sigma_{D}^{\varphi_{1}, x}} e^{-\lambda T_{t}^{(x)}}\left(\hat{\Phi}_{t}^{\varphi_{2}, x}-\frac{\lambda}{c}\right) d T_{t}^{(x)}\right] \\
& -\mathrm{E}^{\infty}\left[\int_{0}^{\sigma_{D}^{\varphi_{1}, x}} e^{-\lambda T_{t}^{(x)}}\left(\hat{\Phi}_{t}^{\varphi_{1}, x}-\frac{\lambda}{c}\right) d T_{t}^{(x)}\right] \\
= & \left(\varphi_{2}-\varphi_{1}\right) \mathrm{E}^{\infty}\left[\int_{0}^{\sigma_{D}^{\varphi_{1}, x}} L_{T_{t}^{(x)}}^{(x)}\left(\hat{X}_{t}^{x}\right)^{2} d t\right] \\
= & \left(\varphi_{2}-\varphi_{1}\right) x^{2} \mathrm{E}^{\infty}\left[\int_{0}^{\sigma_{D}^{\varphi_{1}, x}} \hat{L}_{t}^{(1)}\left(\hat{X}_{t}^{1}\right)^{2} d t\right] \\
\leq & \left(\varphi_{2}-\varphi_{1}\right)\left(x_{0}+\delta\right)^{2} \mathrm{E}^{\infty}\left[\int_{0}^{\sigma_{D}^{\varphi_{0}-\delta, x_{0}-\delta}} \hat{L}_{t}^{(1)}\left(\hat{X}_{t}^{1}\right)^{2} d t\right],
\end{aligned}
$$

where in the second equality we use that $\hat{L}_{t}^{(x)}=L^{(x)} \circ T_{t}^{(x)}=L^{(1)} \circ T_{t}^{(1)}=\hat{L}_{t}^{(1)}$ by (6.19) above and in the final inequality we use (9.1) above. Since the final expectation in (9.7) is finite [as is easily seen from (7.13) above] and not dependent on $\varphi_{1}, \varphi_{2}$ and $x$, we see that (9.5) follows from (9.7) as claimed.

2. To derive (9.6) fix $x_{1}$ and $x_{2}$ in $\left[x_{0}-\delta, x_{0}+\delta\right]$ for $\delta>0$ such that $x_{0}-\delta>0$ with $\varphi \geq 0$ and $x_{0}>0$ given and fixed. Let $\tau_{D}^{\varphi_{0}, x_{1}}$ and $\tau_{D}^{\varphi_{0}, x_{2}}$ denote the optimal stopping time for $\hat{V}\left(\varphi_{0}, x_{1}\right)$ and $\hat{V}\left(\varphi_{0}, x_{2}\right)$ in (4.19) under $\mathrm{P}^{\infty}$, respectively. By the time change (7.1) and the result of Proposition 6, we know that $\sigma_{D}^{\varphi_{0}, x_{1}}=T^{\left(x_{1}\right)}$ 。 $\tau_{D}^{\varphi_{0}, x_{1}}$ and $\sigma_{D}^{\varphi_{0}, x_{2}}=T^{\left(x_{2}\right)} \circ \tau_{D}^{\varphi_{0}, x_{2}}$ are the optimal stopping times for $\hat{V}\left(\varphi_{0}, x_{1}\right)$ and $\hat{V}\left(\varphi_{0}, x_{2}\right)$ in $(7.13)$ under $\mathrm{P}^{\infty}$, respectively. It follows therefore that the following two inequalities are satisfied:

$$
\begin{gathered}
\mathrm{E}^{\infty}\left[\int_{0}^{\sigma_{D}^{\varphi_{0}, x_{2}}} e^{-\lambda T_{t}^{\left(x_{2}\right)}}\left(\hat{\Phi}_{t}^{\varphi_{0}, x_{2}}-\frac{\lambda}{c}\right) d T_{t}^{\left(x_{2}\right)}\right] \\
-\mathrm{E}^{\infty}\left[\int_{0}^{\sigma_{D}^{\varphi_{0}, x_{2}}} e^{-\lambda T_{t}^{\left(x_{1}\right)}}\left(\hat{\Phi}_{t}^{\varphi_{0}, x_{1}}-\frac{\lambda}{c}\right) d T_{t}^{\left(x_{1}\right)}\right] \\
\leq \hat{V}\left(\varphi_{0}, x_{2}\right)-\hat{V}\left(\varphi_{0}, x_{1}\right)
\end{gathered}
$$




$$
\begin{aligned}
\leq & \mathrm{E}^{\infty}\left[\int_{0}^{\sigma_{D}^{\varphi_{0}, x_{1}}} e^{-\lambda T_{t}^{\left(x_{2}\right)}}\left(\hat{\Phi}_{t}^{\varphi_{0}, x_{2}}-\frac{\lambda}{c}\right) d T_{t}^{\left(x_{2}\right)}\right] \\
& -\mathrm{E}^{\infty}\left[\int_{0}^{\sigma_{D}^{\varphi_{0}, x_{1}}} e^{-\lambda T_{t}^{\left(x_{1}\right)}}\left(\hat{\Phi}_{t}^{\varphi_{0}, x_{1}}-\frac{\lambda}{c}\right) d T_{t}^{\left(x_{1}\right)}\right] .
\end{aligned}
$$

For $i=1,2$, we find that

$$
\begin{aligned}
\mid \mathrm{E}^{\infty} & {\left[\int_{0}^{\sigma_{D}^{\varphi_{0}, x_{i}}} e^{-\lambda T_{t}^{\left(x_{2}\right)}}\left(\hat{\Phi}_{t}^{\varphi_{0}, x_{2}}-\frac{\lambda}{c}\right) d T_{t}^{\left(x_{2}\right)}\right] } \\
- & \mathrm{E}^{\infty}\left[\int_{0}^{\sigma_{D}^{\varphi_{0}, x_{i}}} e^{-\lambda T_{t}^{\left(x_{1}\right)}}\left(\hat{\Phi}_{t}^{\varphi_{0}, x_{1}}-\frac{\lambda}{c}\right) d T_{t}^{\left(x_{1}\right)}\right] \mid \\
& \leq \mathrm{E}^{\infty}\left[\int_{0}^{\sigma_{D}^{\varphi_{0}, x_{0}-\delta}}\left|H\left(t ; \varphi_{0}, x_{2}\right)-H\left(t ; \varphi_{0}, x_{1}\right)\right| d t\right],
\end{aligned}
$$

where the (random) function $H$ is defined by

$$
H(t ; \varphi, x)=\left[\hat{L}_{t}^{(1)}\left(\varphi+\lambda \int_{0}^{t} \frac{x^{2}\left(\hat{X}_{s}^{1}\right)^{2}}{e^{-\lambda x^{2} T_{s}^{(1)}} \hat{L}_{s}^{(1)}} d s\right)-\frac{\lambda}{c} e^{-\lambda x^{2} T_{t}^{(1)}}\right] x^{2}\left(\hat{X}_{t}^{1}\right)^{2}
$$

for $t \geq 0$ and $(\varphi, x) \in[0, \infty) \times(0, \infty)$, and in the final inequality of (9.9) we use (9.1) above. Letting $x_{2}-x_{1} \rightarrow 0$ and using the dominated convergence theorem we see that the right-hand side of (9.9) tends to zero, and thus the left-hand side of (9.9) tends to zero as well. Using this fact in (9.8), we see that (9.6) holds as claimed and the proof is complete.

10. Smooth fit. In this section, we prove that the value function $\hat{V}$ from (4.19) satisfies the smooth fit condition at the optimal stopping boundary $b$ from (8.4). A key argument in the proof is based upon the fact that the boundary points are regular for $D$ relative to $(\Phi, X)$ and $(\hat{\Phi}, \hat{X})$ in the sense that

$$
\tau_{D}^{\varphi_{n}, x_{n}} \rightarrow 0 \text { and } \sigma_{D}^{\varphi_{n}, x_{n}} \rightarrow 0
$$

with $\mathrm{P}^{\infty}$-probability one whenever $\left(\varphi_{n}, x_{n}\right)$ from $C$ tends to $(\varphi, x)$ at its boundary $\partial C$ specified by $\varphi=b(x)$ for $x>0$ as $n \rightarrow \infty$. Recall in (10.1) that $\tau_{D}^{\varphi_{n}, x_{n}}$ is the first entry time of $\left(\Phi^{\varphi_{n}, x_{n}}, X^{x_{n}}\right)$ into $D$ and $\sigma_{D}^{\varphi_{n}, x_{n}}$ is the first entry time of ( $\hat{\Phi}^{\varphi_{n}, x_{n}}, \hat{X}^{x_{n}}$ ) into $D$ for $n \geq 1$. It is well known that $(10.1)$ is equivalent to the fact that the first hitting times of $(\Phi, X)$ and $(\hat{\Phi}, \hat{X})$ to $D$ defined by $\tilde{\tau}_{D}=\inf \{t>$ $\left.0 \mid\left(\Phi_{t}, X_{t}\right) \in D\right\}$ and $\tilde{\sigma}_{D}=\inf \left\{t>0 \mid\left(\hat{\Phi}_{t}, \hat{X}_{t}\right) \in D\right\}$ are equal to zero with $\mathrm{P}^{\infty}$. probability one whenever $(\varphi, x)$ belongs to $\partial C$. Given that the time change $t \mapsto T_{t}$ in $(7.1)$ which builds $(\hat{\Phi}, \hat{X})$ from $(\Phi, X)$ is strictly increasing on $[0, \infty)$ we thus see that the boundary points in $\partial C$ are regular for $D$ relative to $(\Phi, X)$ if and only if they are regular relative to $(\hat{\Phi}, \hat{X})$. Recalling that $(\hat{\Phi}, \hat{X})$ solves the system of 
stochastic differential equations $(7.8)+(7.9)$ we can modify the first equation in this system to read

$$
d \tilde{\Phi}_{t}=\gamma \tilde{\Phi}_{t} d \tilde{B}_{t}
$$

upon noting that the (nonnegative) drift term in (7.8) dominates the (zero) drift term in (10.2) so that the comparison result for solutions to multidimensional stochastic differential equations given in [7], Theorem 1, is applicable and yields

$$
\mathrm{P}^{\infty}\left(\tilde{\Phi}_{t}^{\varphi, x} \leq \hat{\Phi}_{t}^{\varphi, x} \text { for all } t \geq 0\right)=1
$$

for $(\varphi, x) \in[0, \infty) \times(0, \infty)$ given and fixed. Since the boundary $x \mapsto b(x)$ between $C$ and $D$ is decreasing on $(0, \infty)$, this shows that the boundary points in $\partial C$ are regular for $D$ relative to $(\hat{\Phi}, \hat{X})$ if they are regular relative to $(\tilde{\Phi}, \hat{X})$. The latter process however is just a pair of geometric Brownian motions (driven by the same Brownian motion) for which [recalling (7.10) above] the regularity at each point $\varphi$ or $x$ for $[\varphi, \infty)$ or $[x, \infty)$ is evident from the regularity of 0 for $[0, \infty)$ relative to standard Brownian motion (with drift). These facts establish (10.1) and equipped with this conclusion we can now state the main result of this section.

Proposition 13 (Smooth fit). For the value function $\hat{V}$ from (4.19), we have

$$
\begin{array}{ll}
\hat{V}_{\varphi}(\varphi, x)=0 & \text { at } \varphi=b(x), \\
\hat{V}_{x}(\varphi, x)=0 & \text { at } \varphi=b(x)
\end{array}
$$

for all $x>0$.

Proof. Let $\varphi=b(x)$ for $x>0$ be given and fixed in the sequel. We first verify that (10.4) holds. This can be done in two steps as follows. We first note that

$$
\liminf _{\varepsilon \downarrow 0} \frac{\hat{V}(\varphi-\varepsilon, x)-\hat{V}(\varphi, x)}{-\varepsilon} \geq 0
$$

due to $\hat{V}(\varphi, x)=0$ and $\hat{V}(\varphi-\varepsilon, x) \leq 0$ for $\varepsilon>0$. We next show that

$$
\limsup _{\varepsilon \downarrow 0} \frac{\hat{V}(\varphi-\varepsilon, x)-\hat{V}(\varphi, x)}{-\varepsilon} \leq 0 .
$$

For this, let $\tau_{\varepsilon}:=\tau_{D}^{\varphi-\varepsilon, x}$ denote the optimal stopping time for $\hat{V}(\varphi-\varepsilon, x)$ in (4.19) under $\mathrm{P}^{\infty}$ with $\varepsilon>0$. Recalling (3.12), we find that

$$
\begin{aligned}
\hat{V}(\varphi & -\varepsilon, x)-\hat{V}(\varphi, x) \\
& \geq \mathrm{E}^{\infty}\left[\int_{0}^{\tau_{\varepsilon}} e^{-\lambda t}\left(\Phi_{t}^{\varphi-\varepsilon, x}-\frac{\lambda}{c}\right) d t\right]-\mathrm{E}^{\infty}\left[\int_{0}^{\tau_{\varepsilon}} e^{-\lambda t}\left(\Phi_{t}^{\varphi, x}-\frac{\lambda}{c}\right) d t\right] \\
& =\mathrm{E}^{\infty}\left[\int_{0}^{\tau_{\varepsilon}}\left(L_{t}^{(x)}\left(\varphi-\varepsilon+\lambda \int_{0}^{t} \frac{d s}{e^{\lambda s} L_{s}^{(x)}}\right)-\frac{\lambda}{c} e^{-\lambda t}\right) d t\right]
\end{aligned}
$$




$$
\begin{aligned}
& -\mathrm{E}^{\infty}\left[\int_{0}^{\tau_{\varepsilon}}\left(L_{t}^{(x)}\left(\varphi+\lambda \int_{0}^{t} \frac{d s}{e^{\lambda s} L_{s}^{(x)}}\right)-\frac{\lambda}{c} e^{-\lambda t}\right) d t\right] \\
= & -\varepsilon \mathrm{E}^{\infty}\left[\int_{0}^{\tau_{\varepsilon}} L_{t}^{(x)} d t\right]
\end{aligned}
$$

for $\varepsilon>0$. Hence, we see that

$$
\frac{\hat{V}(\varphi-\varepsilon, x)-\hat{V}(\varphi, x)}{-\varepsilon} \leq \mathrm{E}^{\infty}\left[\int_{0}^{\tau_{\varepsilon}} L_{t}^{(x)} d t\right]
$$

for $\varepsilon>0$. Letting $\varepsilon \downarrow 0$ and recalling that $\tau_{\varepsilon} \rightarrow 0$ with $\mathrm{P}^{\infty}$-probability one by (10.1) above we see that (10.9) implies (10.7) as claimed. Combining (10.6) and (10.7), we obtain (10.4).

We next verify that (10.5) holds. This can be similarly done in two steps as follows. We first note in parallel to (10.6) that

$$
\liminf _{\varepsilon \downarrow 0} \frac{\hat{V}(\varphi, x-\varepsilon)-\hat{V}(\varphi, x)}{-\varepsilon} \geq 0
$$

due to $\hat{V}(\varphi, x)=0$ and $\hat{V}(\varphi, x-\varepsilon) \leq 0$ for $\varepsilon>0$. We next show in parallel to (10.7) that

$$
\limsup _{\varepsilon \downarrow 0} \frac{\hat{V}(\varphi, x-\varepsilon)-\hat{V}(\varphi, x)}{-\varepsilon} \leq 0 .
$$

For this, let $\sigma_{\varepsilon}:=\sigma_{D}^{\varphi, x-\varepsilon}$ denote the optimal stopping time for $\hat{V}(\varphi, x-\varepsilon)$ in (7.13) under $\mathrm{P}^{\infty}$ with $\varepsilon>0$. Recalling the definition of the (random) function $H$ in (9.10) we find by the mean value theorem that

$$
\begin{aligned}
\hat{V}(\varphi, x-\varepsilon)-\hat{V}(\varphi, x) \geq & \mathrm{E}^{\infty}\left[\int_{0}^{\sigma_{\varepsilon}} e^{-\lambda T_{t}^{(x-\varepsilon)}}\left(\hat{\Phi}_{t}^{\varphi, x-\varepsilon}-\frac{\lambda}{c}\right) d T_{t}^{(x-\varepsilon)}\right] \\
& -\mathrm{E}^{\infty}\left[\int_{0}^{\sigma_{\varepsilon}} e^{-\lambda T_{t}^{(x)}}\left(\hat{\Phi}_{t}^{\varphi, x}-\frac{\lambda}{c}\right) d T_{t}^{(x)}\right] \\
= & \mathrm{E}^{\infty}\left[\int_{0}^{\sigma_{\varepsilon}}(H(t ; \varphi, x-\varepsilon)-H(t ; \varphi, x)) d t\right] \\
= & -\varepsilon \mathrm{E}^{\infty}\left[\int_{0}^{\sigma_{\varepsilon}} H_{x}\left(t ; \varphi, y_{\varepsilon}\right) d t\right]
\end{aligned}
$$

with some $y_{\varepsilon} \in(x-\varepsilon, x)$ for $\varepsilon>0$. Hence, we see that

$$
\frac{\hat{V}(\varphi, x-\varepsilon)-\hat{V}(\varphi, x)}{-\varepsilon} \leq \mathrm{E}^{\infty}\left[\int_{0}^{\sigma_{\varepsilon}} H_{x}\left(t ; \varphi, y_{\varepsilon}\right) d t\right]
$$

for $\varepsilon>0$. Letting $\varepsilon \downarrow 0$ and recalling that $\sigma_{\varepsilon} \rightarrow 0$ with $\mathrm{P}^{\infty}$-probability one by (10.1) above we see that (10.13) implies (10.11) as claimed. Combining (10.10) and (10.11), we obtain (10.5) and the proof is complete. 
11. Regularity of the value function. In this section, we refine the smooth fit results from the previous section and establish a global $C^{1}$ regularity of the value function $\hat{V}$ from (4.19). A key argument in the proof is still based upon the fact that the boundary points are regular for $D$ relative to $(\Phi, X)$ and $(\hat{\Phi}, \hat{X})$ in the sense of (10.1) above. Moreover, recalling reduction of the process $(\Phi, X)$ to the process $(U, \Phi)$ established in Section 6 where $U$ is of bounded variation, we will see that this enables us to extend the global $C^{1}$-regularity to a local $C^{2}$ regularity of $\tilde{V}$ with respect to the second argument up to the optimal stopping boundary within the continuation set (the one within the stopping set being evident). These regularity results play an important role when deriving continuity of the optimal stopping boundary in Section 12 and when applying the local time-space formula from [13] to derive a nonlinear Fredholm integral equation for the optimal stopping boundary in Section 14.

Proposition 14 ( $C^{1}$ regularity). For the value function $\hat{V}$ from (4.19), we have

$$
\begin{aligned}
& (\varphi, x) \mapsto \hat{V}_{\varphi}(\varphi, x) \text { is continuous on }[0, \infty) \times(0, \infty) \\
& (\varphi, x) \mapsto \hat{V}_{x}(\varphi, x) \text { is continuous on }[0, \infty) \times(0, \infty)
\end{aligned}
$$

PROOF. Standard results on partial differential equations of parabolic type (cf. [11], Chapter V) combined with Itô's formula and the optional sampling theorem (cf. [15], pape 131) show that $\hat{V}$ is $C^{2}$ on $C$. Since $\hat{V}$ equals zero on $D$, we thus see that it is enough to prove (11.1) and (11.2) at the optimal stopping boundary. Let us therefore fix $(\varphi, x) \in[0, \infty) \times(0, \infty)$ in the sequel such that $\varphi=b(x)$.

1. We first verify that (11.1) holds. This can be done in two steps as follows. Take any sequence $\left(\varphi_{n}, x_{n}\right) \rightarrow(\varphi, x)$ as $n \rightarrow \infty$ upon recalling that $\varphi=b(x)$. Since $\hat{V}\left(\varphi_{n}, x_{n}\right)=0$ for $\left(\varphi_{n}, x_{n}\right) \in D$ and we have derived (10.4) at $\varphi=b(x)$, it is no restriction to assume that $\left(\varphi_{n}, x_{n}\right) \in C$ for all $n \geq 1$. Thus, to establish (11.1) it is enough to verify that

$$
\lim _{n \rightarrow \infty} \hat{V}_{\varphi}\left(\varphi_{n}, x_{n}\right)=0
$$

For this, first note that

$$
\liminf _{n \rightarrow \infty} \hat{V}_{\varphi}\left(\varphi_{n}, x_{n}\right)=\liminf _{n \rightarrow \infty} \lim _{h \downarrow 0} \frac{\hat{V}\left(\varphi_{n}-h, x_{n}\right)-\hat{V}\left(\varphi_{n}, x_{n}\right)}{-h} \geq 0
$$

since $\varphi \mapsto \hat{V}(\varphi, x)$ is increasing on $[0, \infty)$ for every $x>0$ given and fixed by (8.5) above. Moreover, we next show that

$$
\limsup _{n \rightarrow \infty} \hat{V}_{\varphi}\left(\varphi_{n}, x_{n}\right)=\limsup _{n \rightarrow \infty} \lim _{h \downarrow 0} \frac{\hat{V}\left(\varphi_{n}-h, x_{n}\right)-\hat{V}\left(\varphi_{n}, x_{n}\right)}{-h} \leq 0 .
$$


For this, note from the first identity in (11.5) that we can choose subsequences $\left(\varphi_{n_{k}}\right)_{k \geq 1},\left(x_{n_{k}}\right)_{k \geq 1}$ and $\left(h_{k}\right)_{k \geq 1}$ such that

$$
\limsup _{n \rightarrow \infty} \hat{V}_{\varphi}\left(\varphi_{n}, x_{n}\right)=\lim _{k \rightarrow \infty} \frac{\hat{V}\left(\varphi_{n_{k}}-h_{k}, x_{n_{k}}\right)-\hat{V}\left(\varphi_{n_{k}}, x_{n_{k}}\right)}{-h_{k}}
$$

with $\left(\varphi_{n_{k}}-h_{k}, x_{n_{k}}\right) \rightarrow(\varphi, x)$ as $k \rightarrow \infty$. Let $\tau_{k}:=\tau_{D}^{\varphi_{n_{k}}-h_{k}, x_{n_{k}}}$ denote the optimal stopping time for $\hat{V}\left(\varphi_{n_{k}}-h_{k}, x_{n_{k}}\right)$ under $\mathrm{P}^{\infty}$ with $k \geq 1$. Recalling (3.12), we find in the same way as in (10.8) above that

$$
\hat{V}\left(\varphi_{n_{k}}-h_{k}, x_{n_{k}}\right)-\hat{V}\left(\varphi_{n_{k}}, x_{n_{k}}\right) \geq-h_{k} \mathrm{E}^{\infty}\left[\int_{0}^{\tau_{k}} L_{t}^{\left(x_{n_{k}}\right)} d t\right]
$$

for $k \geq 1$. Using the time change (7.1) and setting $\sigma_{k}=A_{\tau_{k}}$, we find in the same way as in (9.7) above that

$$
\begin{aligned}
\mathrm{E}^{\infty}\left[\int_{0}^{\tau_{k}} L_{t}^{\left(x_{n_{k}}\right)} d t\right] & =\mathrm{E}^{\infty}\left[\int_{0}^{\sigma_{k}} \hat{L}_{t}^{(1)}\left(\hat{X}_{t}^{x_{n_{k}}}\right)^{2} d t\right] \\
& =x_{n_{k}}^{2} \mathrm{E}^{\infty}\left[\int_{0}^{\sigma_{k}} \hat{L}_{t}^{(1)}\left(\hat{X}_{t}^{1}\right)^{2} d t\right]
\end{aligned}
$$

for $k \geq 1$. Combining (11.7) and (11.8), we see that

$$
\frac{\hat{V}\left(\varphi_{n_{k}}-h_{k}, x_{n_{k}}\right)-\hat{V}\left(\varphi_{n_{k}}, x_{n_{k}}\right)}{-h_{k}} \leq x_{n_{k}}^{2} \mathrm{E}^{\infty}\left[\int_{0}^{\sigma_{k}} \hat{L}_{t}^{(1)}\left(\hat{X}_{t}^{1}\right)^{2} d t\right]
$$

for $k \geq 1$. Letting $k \rightarrow \infty$ and recalling that $\tau_{k} \rightarrow 0$ and $\sigma_{k} \rightarrow 0$ with $\mathrm{P}^{\infty}$ probability one by (10.1) above, we see by the dominated convergence theorem that (11.9) combined with (11.6) implies (11.5) as claimed. Combining (11.4) and (11.5), we obtain (11.3), and hence (11.1) follows as explained above.

2. We next verify that (11.2) holds. This can be similarly done in two steps as follows. Recall that to establish (11.2) it is enough to verify that

$$
\lim _{n \rightarrow \infty} \hat{V}_{x}\left(\varphi_{n}, x_{n}\right)=0 .
$$

For this, we first claim in parallel to (11.4) that

$$
\liminf _{n \rightarrow \infty} \hat{V}_{x}\left(\varphi_{n}, x_{n}\right)=\liminf _{n \rightarrow \infty} \lim _{h \downarrow 0} \frac{\hat{V}\left(\varphi_{n}, x_{n}-h\right)-\hat{V}\left(\varphi_{n}, x_{n}\right)}{-h} \geq 0 .
$$

To derive this, note from the first identity in (11.11) that we can choose subsequences $\left(\varphi_{n_{k}}\right)_{k \geq 1},\left(x_{n_{k}}\right)_{k \geq 1}$ and $\left(h_{k}\right)_{k \geq 1}$ such that

$$
\liminf _{n \rightarrow \infty} \hat{V}_{x}\left(\varphi_{n}, x_{n}\right)=\lim _{k \rightarrow \infty} \frac{\hat{V}\left(\varphi_{n_{k}}, x_{n_{k}}-h_{k}\right)-\hat{V}\left(\varphi_{n_{k}}, x_{n_{k}}\right)}{-h_{k}}
$$


with $\left(\varphi_{n_{k}}, x_{n_{k}}-h_{k}\right) \rightarrow(\varphi, x)$ as $k \rightarrow \infty$. Let $\sigma_{k}:=\sigma_{D}^{\varphi_{n_{k}}, x_{n_{k}}}$ denote the optimal stopping time for $\hat{V}\left(\varphi_{n_{k}}, x_{n_{k}}\right)$ in (7.13) under $\mathrm{P}^{\infty}$ with $k \geq 1$. Recalling the definition of the (random) function $H$ in (9.10) we find by the mean value theorem in the same way as in (10.12) above that we have

$$
\begin{aligned}
& \hat{V}\left(\varphi_{n_{k}}, x_{n_{k}}-h_{k}\right)-\hat{V}\left(\varphi_{n_{k}}, x_{n_{k}}\right) \\
& \quad \leq \mathrm{E}^{\infty}\left[\int_{0}^{\sigma_{k}}\left(H\left(t ; \varphi_{n_{k}}, x_{n_{k}}-h_{k}\right)-H\left(t ; \varphi_{n_{k}}, x_{n_{k}}\right)\right) d t\right] \\
& \quad=-h_{k} \mathrm{E}^{\infty}\left[\int_{0}^{\sigma_{k}} H_{x}\left(t ; \varphi_{n_{k}}, y_{k}\right) d t\right]
\end{aligned}
$$

with some $y_{k} \in\left(x_{n_{k}}-h_{k}, x_{n_{k}}\right)$ for $k \geq 1$. Hence, we see that

$$
\frac{\hat{V}\left(\varphi_{n_{k}}, x_{n_{k}}-h_{k}\right)-\hat{V}\left(\varphi_{n_{k}}, x_{n_{k}}\right)}{-h_{k}} \geq \mathrm{E}^{\infty}\left[\int_{0}^{\sigma_{k}} H_{x}\left(t ; \varphi_{n_{k}}, y_{k}\right) d t\right]
$$

for $k \geq 1$. Letting $k \rightarrow \infty$ and recalling that $\sigma_{k} \rightarrow 0$ with $\mathrm{P}^{\infty}$-probability one we see by the dominated convergence theorem that (11.14) combined with (11.12) implies (11.11) as claimed.

We next claim in parallel to (11.5) that

$$
\limsup _{n \rightarrow \infty} \hat{V}_{x}\left(\varphi_{n}, x_{n}\right)=\limsup _{n \rightarrow \infty} \lim _{h \downarrow 0} \frac{\hat{V}\left(\varphi_{n}, x_{n}-h\right)-\hat{V}\left(\varphi_{n}, x_{n}\right)}{-h} \leq 0 .
$$

For this, we can proceed similarly to the arguments given above and choose subsequences $\left(\varphi_{n_{k}}\right)_{k \geq 1},\left(x_{n_{k}}\right)_{k \geq 1}$ and $\left(h_{k}\right)_{k \geq 1}$ such that

$$
\limsup _{n \rightarrow \infty} \hat{V}_{x}\left(\varphi_{n}, x_{n}\right)=\lim _{k \rightarrow \infty} \frac{\hat{V}\left(\varphi_{n_{k}}, x_{n_{k}}-h_{k}\right)-\hat{V}\left(\varphi_{n_{k}}, x_{n_{k}}\right)}{-h_{k}}
$$

with $\left(\varphi_{n_{k}}, x_{n_{k}}-h_{k}\right) \rightarrow(\varphi, x)$ as $k \rightarrow \infty$. Let $\sigma_{k}:=\sigma_{D}^{\varphi_{n_{k}}, x_{n_{k}}-h_{k}}$ denote the optimal stopping time for $\hat{V}\left(\varphi_{n_{k}}-h_{k}, x_{n_{k}}\right)$ in (7.13) under $\mathrm{P}^{\infty}$ with $k \geq 1$. Then in the same way as in (11.13) we find that

$$
\begin{aligned}
& \hat{V}\left(\varphi_{n_{k}}, x_{n_{k}}-h_{k}\right)-\hat{V}\left(\varphi_{n_{k}}, x_{n_{k}}\right) \\
& \quad \geq \mathrm{E}^{\infty}\left[\int_{0}^{\sigma_{k}}\left(H\left(t ; \varphi_{n_{k}}, x_{n_{k}}-h_{k}\right)-H\left(t ; \varphi_{n_{k}}, x_{n_{k}}\right)\right) d t\right] \\
& \quad=-h_{k} \mathrm{E}^{\infty}\left[\int_{0}^{\sigma_{k}} H_{x}\left(t ; \varphi_{n_{k}}, y_{k}\right) d t\right]
\end{aligned}
$$

with some $y_{k} \in\left(x_{n_{k}}-h_{k}, x_{n_{k}}\right)$ for $k \geq 1$. Hence, we see that

$$
\frac{\hat{V}\left(\varphi_{n_{k}}, x_{n_{k}}-h_{k}\right)-\hat{V}\left(\varphi_{n_{k}}, x_{n_{k}}\right)}{-h_{k}} \leq \mathrm{E}^{\infty}\left[\int_{0}^{\sigma_{k}} H_{x}\left(t ; \varphi_{n_{k}}, y_{k}\right) d t\right]
$$


for $k \geq 1$. Letting $k \rightarrow \infty$ and recalling that $\sigma_{k} \rightarrow 0$ with $\mathrm{P}^{\infty}$-probability one we see by the dominated convergence theorem that (11.18) combined with (11.16) implies (11.15) as claimed. Combining (11.11) and (11.15), we obtain (11.10) as needed and the proof is complete.

1. The result of Proposition 4 and Corollary 5 showed that the optimal stopping problem (4.19) for the strong Markov process $(\Phi, X)$ can be considered as the optimal stopping problem

$$
\tilde{V}(u, \varphi)=\inf _{\tau} \mathrm{E}_{u, \varphi}^{\infty}\left[\int_{0}^{\tau} e^{-\lambda t}\left(\Phi_{t}-\frac{\lambda}{c}\right) d t\right]
$$

for the strong Markov process $(U, \Phi)$ with $\mathrm{P}_{u, \varphi}^{\infty}\left(\left(U_{0}, \Phi_{0}\right)=(u, \varphi)\right)=1$ for $(u, \varphi) \in \mathbb{R} \times[0, \infty)$ and the infimum in (11.19) is taken over all stopping times $\tau$ of $(U, \Phi)$. The two optimal stopping problems are equivalent and the transformation (6.14) maps the optimal stopping boundary $b$ in the problem (4.19) to the optimal stopping boundary $\tilde{b}$ in the problem (11.19) that is determined as follows. Recalling from (6.14) that $x^{\gamma}=\varphi e^{u}$, or equivalently $\varphi=e^{-u} x^{\gamma}$, and using the results of Corollary 8 and Propositions 9 and 10, we see that for every $u \in \mathbb{R}$ there exists a unique $x>0$ such that

$$
\varphi:=b(x)=e^{-u} x^{\gamma}=: \tilde{b}(u) .
$$

The global one-to-one nature of this transformation shows that (11.20) defines the optimal stopping boundary in (11.19) so that its continuation and stopping sets are given by

$$
\begin{aligned}
& \tilde{C}=\{(u, \varphi) \in \mathbb{R} \times[0, \infty) \mid \varphi<\tilde{b}(u)\}, \\
& \tilde{D}=\{(u, \varphi) \in \mathbb{R} \times[0, \infty) \mid \varphi \geq \tilde{b}(u)\} .
\end{aligned}
$$

Moreover, from the results of Corollary 8 and Propositions 9 and 10 we see that

$$
\begin{aligned}
u & \mapsto \tilde{b}(u) \text { is decreasing on } \mathbb{R}, \\
\lim _{u \rightarrow-\infty} \tilde{b}(u) & =\infty, \\
\lim _{u \rightarrow \infty} \tilde{b}(u) & =\frac{\lambda}{c} .
\end{aligned}
$$

From (6.14), we see that the basic relationship between $\hat{V}$ and $\tilde{V}$ is described by

$$
\hat{V}(\varphi, x)=\tilde{V}\left(\log \left(x^{\gamma} / \varphi\right), \varphi\right) \quad \text { and } \quad \tilde{V}(u, \varphi)=\hat{V}\left(\varphi, \varphi^{1 / \gamma} e^{u / \gamma}\right)
$$

for $(\varphi, x) \in[0, \infty) \times(0, \infty)$ and $u \in \mathbb{R}$ where $x^{\gamma}=\varphi e^{u}$ as stated in (6.14) above.

2. The advantage of the optimal stopping problem (11.19) in comparison with the optimal stopping problem (4.19) is that the process $U$ is of bounded variation. We will now see how this enables us to extend the global continuity of the first partial derivatives of $\tilde{V}$ to the continuity of its second partial derivative with respect 
to $\varphi$ up to the optimal stopping boundary $\tilde{b}$ within $\tilde{C}$. For this, let $\operatorname{cl}(\tilde{C})$ denote the (topological) closure of $\tilde{C}$ in $\mathbb{R} \times[0, \infty)$ given by

$$
\operatorname{cl}(\tilde{C})=\{(u, \varphi) \in \mathbb{R} \times[0, \infty) \mid \varphi \leq \tilde{b}(u)\} .
$$

Recall from the result and proof of Proposition 14 that $\hat{V}$ is $C^{1}$ globally and $C^{2}$ on $C$ so that due to (11.26) we see that $\tilde{V}$ is $C^{1}$ globally and $C^{2}$ on $\tilde{C}$. Making use of the optimal stopping problem (11.19) combined with the fact that the process $U$ is of bounded variation we can now extend the latter fact as follows.

COROLlary 15 ( $C^{2}$ regularity). For the value function $\tilde{V}$ from (11.19), we have

$\tilde{V}_{\varphi \varphi}$ admits a continuous extension from $\tilde{C}$ to $\operatorname{cl}(\tilde{C})$.

PROOF. Recalling (11.26), we see from (11.1) and (11.2) that

$$
\tilde{V} \text { is } C^{1} \quad \text { on } \mathbb{R} \times[0, \infty)
$$

as noted above. Moreover, from the Lagrange formulation (11.19) we know that

$$
\mathbb{L}_{U, \Phi} \tilde{V}-\lambda \tilde{V}=-(\varphi-\lambda / c)
$$

in $\tilde{C}$ where $\mathbb{L}_{U, \Phi}$ is the infinitesimal generator of $(U, \Phi)$ given by (6.26) above. Noting that the left-hand side in (11.30) contains only one second partial derivative $\tilde{V}_{\varphi \varphi}$ in addition to $\tilde{V}_{u}$ and $\tilde{V}_{\varphi}$, we see that (11.29) combined with (11.30) implies that (11.28) holds as claimed.

12. Continuity of the optimal stopping boundary. In this section, we prove that the optimal stopping boundary $b$ from (8.4) is continuous. The proof makes use of the fact that its optimal stopping problem (4.19) of $(\Phi, X)$ is equivalent to the optimal stopping problem (11.19) of $(U, \Phi)$ for which the boundary $\tilde{b}$ from (11.20) is optimal. The advantage of the latter problem is that the infinitesimal generator $\mathbb{L}_{U, \Phi}$ from (6.26) contains only one second partial derivative (with respect to the second argument) so that simple techniques based on its dual $\mathbb{L}_{U, \Phi}^{*}$ are directly applicable. This is another consequence of the fact that the infinitesimal generator $\mathbb{L}_{\Phi, X}$ from (5.17) is of parabolic type.

Proposition 16. The mapping $x \mapsto b(x)$ is continuous on $(0, \infty)$.

ProOF. Let us first show that $x \mapsto b(x)$ is right-continuous. For this, fix $x \in(0, \infty)$ and take any sequence $x_{n} \downarrow x$ as $n \rightarrow \infty$. Since $x \mapsto b(x)$ is decreasing on $(0, \infty)$ as established in Corollary 8 , it follows that the right-hand limit $b(x+)$ exists. Because $\left(b\left(x_{n}\right), x_{n}\right) \in D$ for $n \geq 1$ and $D$ is closed this implies that $(b(x+), x) \in D$. Hence, by definition of $b$ in (8.4) we see that $b(x) \leq b(x+)$. Since 
the reverse inequality follows clearly from the fact that $x \mapsto b(x)$ is decreasing on $(0, \infty)$, we see that $b(x+)=b(x)$ as claimed.

Suppose that at some point $x_{*}$ in $(0, \infty)$ the function $b$ makes a jump. Using the transformation $x^{\gamma}=\varphi e^{u}$ originating in (6.14) and recalling the results of Corollary 8 and Propositions 9 and 10, we thus see from (11.20) that at some point $u_{*}$ in $\mathbb{R}$ the function $\tilde{b}$ makes a jump. Fix a point $u_{1}<u_{2}$ close to $u_{2}$ upon setting $u_{2}=u_{*}$, denote $\varphi_{1}=\tilde{b}\left(u_{2}\right)$ and $\varphi_{2}=\tilde{b}\left(u_{2}-\right)$, and consider the rectangle $R=\left[u_{1}, u_{2}\right] \times\left[\varphi_{1}, \varphi_{2}\right]$ with vertices $\left(u_{1}, \varphi_{1}\right),\left(u_{2}, \varphi_{1}\right),\left(u_{1}, \varphi_{2}\right)$ and $\left(u_{2}, \varphi_{2}\right)$. Note from (11.23) and (11.25) that there is no loss of generality in assuming that $\varphi_{1}>\lambda / c$ since otherwise we could formally replace $\varphi_{1}$ by $\left(\varphi_{1}+\varphi_{2}\right) / 2$ in the proof below and still have $\varphi_{1}<\varphi_{2}$ due to the jump of $\tilde{b}$ at $u_{2}$ as needed.

Recall from (6.26) that

$$
\mathbb{L}_{U, \Phi} F=\left[\frac{\beta}{\varphi^{2 / \gamma} e^{\frac{2}{\gamma} u}}-\lambda \frac{1+\varphi}{\varphi}\right] F_{u}+\lambda(1+\varphi) F_{\varphi}+\frac{\gamma^{2}}{2} \frac{\varphi^{2-2 / \gamma}}{e^{\frac{2}{\gamma} u}} F_{\varphi \varphi}
$$

for a smooth function $F=F(u, \varphi)$ with $(u, \varphi) \in \mathbb{R} \times[0, \infty)$. Motivated by particular needs that will become clearer below, define

$$
\begin{aligned}
& f(u)=u-u_{1}, \\
& g(\varphi)=\frac{1}{\varphi_{2}-\varphi_{1}}\left[1+\cos \left(\frac{2 \varphi-\varphi_{1}-\varphi_{2}}{\varphi_{2}-\varphi_{1}} \pi\right)\right]
\end{aligned}
$$

for $u \in\left[u_{1}, u_{2}\right]$ and $\varphi \in\left[\varphi_{1}, \varphi_{2}\right]$, respectively. Note that $f\left(u_{1}\right)=0, f^{\prime}(u)=1$ and $f^{\prime \prime}(u)=0$ for $u \in\left[u_{1}, u_{2}\right]$ and it is easily verified that $g\left(\varphi_{i}\right)=g^{\prime}\left(\varphi_{i}\right)=0$ for $i=1,2$ with $g(\varphi)>0$ for $\varphi \in\left(\varphi_{1}, \varphi_{2}\right)$ and $\int_{\varphi_{1}}^{\varphi_{2}} g(\varphi) d \varphi=1$. Defining

$$
F(u, \varphi)=f(u) g(\varphi)
$$

for $u \in\left[u_{1}, u_{2}\right]$ and $\varphi \in\left[\varphi_{1}, \varphi_{2}\right]$ this shows that

$$
\begin{array}{rlll}
F\left(u, \varphi_{1}\right)=0 & \text { and } & F\left(u, \varphi_{2}\right)=0, \\
F_{\varphi}\left(u, \varphi_{1}\right)=0 & \text { and } & F_{\varphi}\left(u, \varphi_{2}\right)=0, \\
F\left(u_{1}, \varphi\right)=0 & \text { and } & F_{\varphi}\left(u_{1}, \varphi\right)=0
\end{array}
$$

for all $u \in\left(u_{1}, u_{2}\right)$ and $\varphi \in\left(\varphi_{1}, \varphi_{2}\right)$. Moreover, recalling (10.4) and (10.5) together with $(11.26)$ and the fact that $\left(u_{2}, \varphi\right) \in \tilde{D}$, we see that the instantaneous stopping condition and the smooth fit condition in the optimal stopping problem (11.19) read as follows:

$$
\begin{gathered}
\tilde{V}\left(u_{2}, \varphi\right)=0, \\
\tilde{V}_{u}\left(u_{2}, \varphi\right)=0
\end{gathered}
$$

for all $\varphi \in\left(\varphi_{1}, \varphi_{2}\right)$. A lengthy calculation based on a repeated integration by parts that makes use of (12.5)-(12.7) and (12.8) + (12.9) then shows that

$$
\int_{u_{1}}^{u_{2}} \int_{\varphi_{1}}^{\varphi_{2}} F \mathbb{L}_{U, \Phi} \tilde{V} d u d \varphi=\int_{u_{1}}^{u_{2}} \int_{\varphi_{1}}^{\varphi_{2}} \tilde{V} \mathbb{L}_{U, \Phi}^{*} F d u d \varphi
$$


where we set

$$
\begin{aligned}
\mathbb{L}_{U, \Phi}^{*} F= & -\left(\left[\frac{\beta}{\varphi^{2 / \gamma} e^{\frac{2}{\gamma} u}}-\lambda \frac{1+\varphi}{\varphi}\right] F\right)_{u} \\
& -(\lambda(1+\varphi) F)_{\varphi}+\left(\frac{\gamma^{2}}{2} \frac{\varphi^{2-2 / \gamma}}{e^{\frac{2}{\gamma} u}} F\right)_{\varphi \varphi} .
\end{aligned}
$$

Recalling that $\tilde{V}$ solves (11.30) in $\tilde{C}$, we see using (12.10) that

$$
\begin{aligned}
\int_{u_{1}}^{u_{2}} \int_{\varphi_{1}}^{\varphi_{2}}-(\varphi-\lambda / c) F d u d \varphi & =\int_{u_{1}}^{u_{2}} \int_{\varphi_{1}}^{\varphi_{2}}\left(\mathbb{L}_{U, \Phi} \tilde{V}-\lambda \tilde{V}\right) F d u d \varphi \\
& =\int_{u_{1}}^{u_{2}} \int_{\varphi_{1}}^{\varphi_{2}} \tilde{V}\left(\mathbb{L}_{U, \Phi}^{*} F-\lambda F\right) d u d \varphi .
\end{aligned}
$$

To proceed further, we will now estimate the initial and the final expression in (12.12).

First, using (12.4) we find that

$$
\begin{aligned}
\int_{u_{1}}^{u_{2}} \int_{\varphi_{1}}^{\varphi_{2}}-(\varphi-\lambda / c) F d u d \varphi & \leq-\left(\varphi_{1}-\lambda / c\right) \int_{u_{1}}^{u_{2}} \int_{\varphi_{1}}^{\varphi_{2}} f(u) g(\varphi) d u d \varphi \\
& =-\left(\varphi_{1}-\lambda / c\right) \frac{\left(u_{2}-u_{1}\right)^{2}}{2},
\end{aligned}
$$

where we recall that $\varphi_{1}>\lambda / c$ so that the final term is strictly negative.

Second, note that the mean value theorem together with the continuous/smooth fit $(12.8)+(12.9)$ and the regularity fact (11.29) imply the existence of a point $v \in\left(u, u_{2}\right)$ such that

$$
|\tilde{V}(u, \varphi)|=\left|\tilde{V}(u, \varphi)-\tilde{V}\left(u_{2}, \varphi\right)\right|=\left|\tilde{V}_{u}(v, \varphi)\right|\left|u-u_{2}\right| \leq \varepsilon\left(u_{2}-u\right)
$$

for all $(u, \varphi) \in\left(u_{1}, u_{2}\right) \times\left(\varphi_{1}, \varphi_{2}\right)$ with $u_{2}-u_{1}<\delta$.

Third, it is easily seen from (12.4) with (12.2) + (12.3) and (12.11) that

$$
\left|\mathbb{L}_{U, \Phi}^{*} F-\lambda F\right| \leq C
$$

on $\left[u_{1}, u_{2}\right] \times\left[\varphi_{1}, \varphi_{2}\right]$ for some $C>0$ large enough. Using (12.14) and (12.15), we find that

$$
\begin{aligned}
\int_{u_{1}}^{u_{2}} \int_{\varphi_{1}}^{\varphi_{2}} \tilde{V}\left(\mathbb{L}_{U, \Phi}^{*} F-\lambda F\right) d u d \varphi & \geq-\int_{u_{1}}^{u_{2}} \int_{\varphi_{1}}^{\varphi_{2}}|\tilde{V}|\left|\mathbb{L}_{U, \Phi}^{*} F-\lambda F\right| d u d \varphi \\
& \geq-\varepsilon C\left(\varphi_{2}-\varphi_{1}\right) \int_{u_{1}}^{u_{2}}\left(u_{2}-u\right) d u \\
& =-\varepsilon C\left(\varphi_{2}-\varphi_{1}\right) \frac{\left(u_{2}-u_{1}\right)^{2}}{2} .
\end{aligned}
$$


Combining (12.12), (12.13) and (12.16), we get

$$
-\varepsilon C\left(\varphi_{2}-\varphi_{1}\right) \frac{\left(u_{2}-u_{1}\right)^{2}}{2} \leq-\left(\varphi_{1}-\lambda / c\right) \frac{\left(u_{2}-u_{1}\right)^{2}}{2}
$$

for all $(u, \varphi) \in\left(u_{1}, u_{2}\right) \times\left(\varphi_{1}, \varphi_{2}\right)$ with $u_{2}-u_{1}<\delta$. This implies that $\varepsilon \geq\left(\varphi_{1}-\right.$ $\lambda / c) /\left(\varphi_{2}-\varphi_{1}\right)$ for all $\varepsilon>0$ and letting $\varepsilon \downarrow 0$ we get that $\varphi_{1}=\lambda / c$ which is a contradiction with the fact that $\varphi_{1}>\lambda / c$. It follows therefore that $\tilde{b}$ does not make a jump, and hence the same fact is true for $b$ as claimed. This completes the proof.

13. Free-boundary problem. In this section, we derive the free-boundary problems that stand in one-to-one correspondence with the optimal stopping problems (4.19) and (11.19), respectively. The two free-boundary problems are equivalent and the latter problem can be seen as a canonical reformulation of the former problem by means of the diffeomorphic transformation (6.14). Using results derived in the previous sections, we show that the value functions and their optimal stopping boundaries $(\hat{V}, b)$ and $(\tilde{V}, \tilde{b})$ from (4.19) and (11.19) solve the freeboundary problems, respectively. This establishes the existence of a solution. Its uniqueness in natural classes of functions will follow from a more general uniqueness result derived in Section 14 below. This will also yield a triple-integral representation for the value function $\hat{V}$ expressed in terms of the optimal stopping boundary $b$. A similar integral representation also holds for the value function $\tilde{V}$ expressed in terms of the optimal stopping boundary $\tilde{b}$ but we will not state it explicitly.

1. We first consider the optimal stopping problem (4.19) where the strong Markov process $(\Phi, X)$ solves the system of stochastic differential equations $(4.20)+(4.21)$ under the measure $\mathrm{P}_{\varphi, x}^{\infty}$ with $(\varphi, x) \in[0, \infty) \times(0, \infty)$. Recalling that the infinitesimal generator $\mathbb{L}_{\Phi, X}$ of $(\Phi, X)$ is given by (5.17) and relying on other properties of $\hat{V}$ and $b$ derived above, we are naturally led to formulate the following free-boundary problem for finding $\hat{V}$ and $b$ :

$$
\begin{gathered}
\lambda(1+\varphi) \hat{V}_{\varphi}+\frac{\delta_{0}-1}{2 x} \hat{V}_{x}+\gamma \frac{\varphi}{x} \hat{V}_{\varphi x}+\frac{\gamma^{2}}{2} \frac{\varphi^{2}}{x^{2}} \hat{V}_{\varphi \varphi}+\frac{1}{2} \hat{V}_{x x}-\lambda V \\
=-\left(\varphi-\frac{\lambda}{c}\right) \quad \text { in } C,
\end{gathered}
$$

where the (continuation) set $C$ and the (stopping) set $D$ are given by

$$
\begin{array}{ll}
\hat{V}(\varphi, x)=0 & \text { for }(\varphi, x) \in D \text { (instantaneous stopping), } \\
\hat{V}_{\varphi}(\varphi, x)=0 & \text { for } \varphi=b(x) \text { with } x>0 \text { (smooth fit), } \\
\hat{V}_{x}(\varphi, x)=0 & \text { for } \varphi=b(x) \text { with } x>0 \text { (smooth fit), }
\end{array}
$$

$$
\begin{aligned}
& C=\{(\varphi, x) \in[0, \infty) \times(0, \infty) \mid \varphi<b(x)\}, \\
& D=\{(\varphi, x) \in[0, \infty) \times(0, \infty) \mid \varphi \geq b(x)\} .
\end{aligned}
$$


Clearly, the global condition (13.2) can be replaced by the local condition $\hat{V}(\varphi, x)=0$ for $\varphi=b(x)$ with $x>0$ so that the free-boundary problem (13.1)(13.4) needs to be considered on the closure of $C$ only (the extension of $\hat{V}$ to $D$ as zero being then evident). Moreover, the free-boundary problem (13.1)-(13.4) can be reformulated in a number of ways. Among these, we could consider the Mayer reformulation (Section 5) or the time-change reformulation (Section 7), as well as various combinations of these and other possibilities [such as the one corresponding to the Markov process $(U, X)$ which we do not consider]. All these reformulations of the free-boundary problem (13.1)-(13.4) are equivalent and among them we will only single out a canonical reformulation corresponding to the parabolic PDE reduction (Section 6).

2. We next consider the optimal stopping problem (11.19) where the strong Markov process $(U, \Phi)$ solves the system of stochastic differential equations $(6.16)+(6.17)$ under the measure $\mathrm{P}_{u, \varphi}^{\infty}$ with $(u, \varphi) \in \mathbb{R} \times[0, \infty)$. Recalling that the infinitesimal generator $\mathbb{L}_{U, \Phi}$ of $(U, \Phi)$ is given by (6.26) and relying on the connection between $(U, \Phi)$ and $(\Phi, X)$ realised through (6.14) combined with other properties of $\tilde{V}$ and $\tilde{b}$ derived above, we are naturally led to formulate the following free-boundary problem for finding $\tilde{V}$ and $\tilde{b}$ :

$$
\begin{aligned}
& {\left[\frac{\beta}{\varphi^{2 / \gamma} e^{\frac{2}{\gamma} u}}-\lambda \frac{1+\varphi}{\varphi}\right] \tilde{V}_{u}+\lambda(1+\varphi) \tilde{V}_{\varphi}+\frac{\gamma^{2}}{2} \frac{\varphi^{2-2 / \gamma}}{e^{\frac{2}{\gamma} u}} \tilde{V}_{\varphi \varphi}-\lambda \tilde{V}} \\
& =-\left(\varphi-\frac{\lambda}{c}\right) \quad \text { in } \tilde{C},
\end{aligned}
$$

$$
\begin{array}{ll}
\tilde{V}(u, \varphi)=0 & \text { for }(u, \varphi) \in \tilde{D} \text { (instantaneous stopping), } \\
\tilde{V}_{u}(u, \varphi)=0 & \text { for } \varphi=\tilde{b}(u) \text { with } u \in \mathbb{R} \text { (smooth fit), } \\
\tilde{V}_{\varphi}(u, \varphi)=0 & \text { for } \varphi=\tilde{b}(u) \text { with } u \in \mathbb{R} \text { (smooth fit), }
\end{array}
$$

where the (continuation) set $\tilde{C}$ and the (stopping) set $\tilde{D}$ are given by

$$
\begin{aligned}
& \tilde{C}=\{(u, \varphi) \in \mathbb{R} \times[0, \infty) \mid \varphi<\tilde{b}(x)\}, \\
& \tilde{D}=\{(u, \varphi) \in \mathbb{R} \times[0, \infty) \mid \varphi \geq \tilde{b}(x)\} .
\end{aligned}
$$

Clearly, the global condition (13.8) can be replaced by the local condition $\tilde{V}(u, \varphi)=0$ for $\varphi=\tilde{b}(u)$ with $u \in \mathbb{R}$ so that the free-boundary problem (13.7)(13.10) needs to be considered on the closure of $\tilde{C}$ only (the extension of $\tilde{V}$ to $\tilde{D}$ as zero being then evident).

3. To formulate the existence and uniqueness result for the problem (13.1)(13.4), we let $\mathcal{C}$ denote the class of pairs of functions $(F, a)$ such that

(13.13) $F$ belongs to $C^{1}\left(\bar{C}_{a}\right) \cap C^{2}\left(C_{a}\right)$ and is bounded on $[0, \infty) \times(0, \infty)$, (13.14) $a$ is continuous and decreasing on $(0, \infty)$ with $a(x) \geq \lambda / c$ for $x>0$, where we set $C_{a}=\{(\varphi, x) \in[0, \infty) \times(0, \infty) \mid \varphi<a(x)\}$. 
THEOREM 17. The free-boundary problem (13.1)-(13.4) has a unique solution $(\hat{V}, b)$ in the class $\mathcal{C}$ where $\hat{V}$ is given by (4.19) and $b$ is defined in (8.4).

PROOF. For the value function $\hat{V}$ from (4.19) and its optimal stopping boundary $b$ from (8.4), it was established in Proposition 14 that $\hat{V}$ belongs to $C^{1}(\bar{C}) \cap$ $C^{2}(C)$ and in Proposition 16 with Corollary 8 that $b$ is continuous and decreasing on $(0, \infty)$. Since $\hat{V}$ is bounded this shows that the pair $(\hat{V}, b)$ belongs to the class $\mathcal{C}$. Moreover, from the Lagrange formulation (4.19) we know that $\hat{V}$ solves (13.1) and from Proposition 13 we know that $\hat{V}$ satisfies (13.3) and (13.4). Since $\hat{V}$ evidently satisfies (13.2) this shows that the pair $(\hat{V}, b)$ is a solution to the free-boundary problem (13.1)-(13.4) in the class $\mathcal{C}$. To derive uniqueness of the solution, we will first see in the next section that any solution $(F, a)$ to (13.1)(13.4) in the class $\mathcal{C}$ admits a closed triple-integral representation for $F$ expressed in terms of $a$, which in turn solves a nonlinear Fredholm integral equation, and we will then show that this equation cannot have other solutions satisfying (13.14) above. Putting these facts together shows that there could be no more than one solution to (13.1)-(13.4) in the class $\mathcal{C}$ as claimed.

4. To formulate the existence and uniqueness result for the problem (13.7)(13.10), we let $\tilde{\mathcal{C}}$ be defined in exactly the same way as $\mathcal{C}$ above with the domains $[0, \infty) \times(0, \infty)$ and $(0, \infty)$ being replaced by the domains $\mathbb{R} \times[0, \infty)$ and $\mathbb{R}$, respectively.

COROLLARY 18. The free-boundary problem (13.7)-(13.10) has a unique solution $(\tilde{V}, \tilde{b})$ in the class $\tilde{\mathcal{C}}$ where $\tilde{V}$ is given by (11.19) and $\tilde{b}$ is defined in (11.20).

PROOF. This follows from Theorem 17 using the fact that the value function $\tilde{V}$ from (11.19) and its optimal stopping boundary $\tilde{b}$ from (11.20) are a canonical reformulation of the value function $\hat{V}$ from (4.19) and its optimal stopping boundary $b$ from (8.4) obtained by means of the diffeomorphic transformation (6.14) as explained in Section 6 above.

14. Nonlinear integral equation. In this section, we show that the optimal stopping boundary $b$ from (8.4) can be characterised as the unique solution to a nonlinear Fredholm integral equation (Theorem 19). This also yields a closed triple-integral representation of the value function $\hat{V}$ from (4.19) expressed in terms of the optimal stopping boundary $b$. Analogous results also hold for the optimal stopping boundary $\tilde{b}$ from (11.20) and the value function $\tilde{V}$ from (11.19) but we will not state them explicitly. As a consequence of the existence and uniqueness result for the nonlinear Fredholm integral equation we also obtain uniqueness of the solution to the free-boundary problems (13.1)-(13.4) and (13.7)-(13.10) as explained in the proofs of Theorem 17 and Corollary 18 above. Finally, collecting the results derived throughout the paper we conclude our exposition at the end of this section by disclosing the solution to the initial problem (Corollary 20). 
1. To formulate the theorem below, let $p$ denote the transition density of the (time-homogeneous) Markov process $(\Phi, X)$ under $\mathrm{P}^{\infty}$ in the sense that

$$
\mathrm{P}_{\varphi, x}^{\infty}\left(\Phi_{t} \leq \psi, X_{t} \leq y\right)=\int_{0}^{\psi} \int_{0}^{y} p(t ; \varphi, x ; \eta, z) d \eta d z
$$

for $t>0$ with $(\varphi, x)$ and $(\psi, x)$ in $[0, \infty) \times(0, \infty)$. The function $p$ is characterised as the unique nonnegative solution to the Kolmogorov backward equation

$$
\begin{aligned}
p_{t}(t ; \varphi, x ; \eta, z) & =\mathbb{L}_{\Phi, X}(p)(t ; \varphi, x ; \eta, z), \\
p(0+; \varphi, x ; \eta, z) & =\delta_{(\varphi, x)}(\eta, z) \quad \text { (weakly) }
\end{aligned}
$$

satisfying $\int_{0}^{\infty} \int_{0}^{\infty} p(t ; \varphi, x ; \eta, z) d \eta d z=1$ for $t>0$ with $(\varphi, x)$ and $(\eta, z)$ in $[0, \infty) \times(0, \infty)$ (cf. [6]) where we recall that $\mathbb{L}_{\Phi, X}$ is given in (5.17) above and $\delta_{(\varphi, x)}$ denotes the Dirac measure at $(\varphi, x)$. The initial value problem $(14.2)+$ (14.3) can be used to determine $p$.

Having $p$, we can then evaluate the expression of interest appearing in the statement of the theorem below as follows:

$$
\begin{aligned}
\int_{0}^{\infty} & e^{-\lambda t} \mathrm{E}_{\varphi, x}^{\infty}\left[H\left(\Phi_{t}, X_{t}\right) I\left(\Phi_{t}<b\left(X_{t}\right)\right)\right] d t \\
& =\int_{0}^{\infty} \int_{0}^{\infty} \int_{0}^{b(y)} e^{-\lambda t} H(\psi, y) p(t ; \varphi, x ; \psi, y) d \psi d y d t
\end{aligned}
$$

for $(\varphi, x)$ in $[0, \infty) \times(0, \infty)$ where we recall that $H$ is defined in $(5.16)$ above.

THEOREM 19 (Existence and uniqueness). The optimal stopping boundary $b$ in the problem (4.19) can be characterised as the unique solution to the nonlinear integral equation

$$
\int_{0}^{\infty} \int_{0}^{\infty} \int_{0}^{b(y)} e^{-\lambda t} H(\psi, y) p(t ; b(x), x ; \psi, y) d \psi d y d t=0
$$

in the class of continuous and decreasing functions $x \mapsto b(x)$ satisfying $b(x) \geq$ $\lambda / c$ for $x>0$. The value function $\hat{V}$ in the problem (4.19) admits the following representation:

$$
\hat{V}(\varphi, x)=\int_{0}^{\infty} \int_{0}^{\infty} \int_{0}^{b(y)} e^{-\lambda t} H(\psi, y) p(t ; \varphi, x ; \psi, y) d \psi d y d t
$$

for $(\varphi, x)$ in $[0, \infty) \times(0, \infty)$. The optimal stopping time in the problem (4.19) is given by

$$
\tau_{b}=\inf \left\{t \geq 0 \mid \Phi_{t} \geq b\left(X_{t}\right)\right\}
$$

under $\mathrm{P}_{\varphi, x}^{\infty}$ with $(\varphi, x)$ in $[0, \infty) \times(0, \infty)$ given and fixed (see Figure 3 above). 


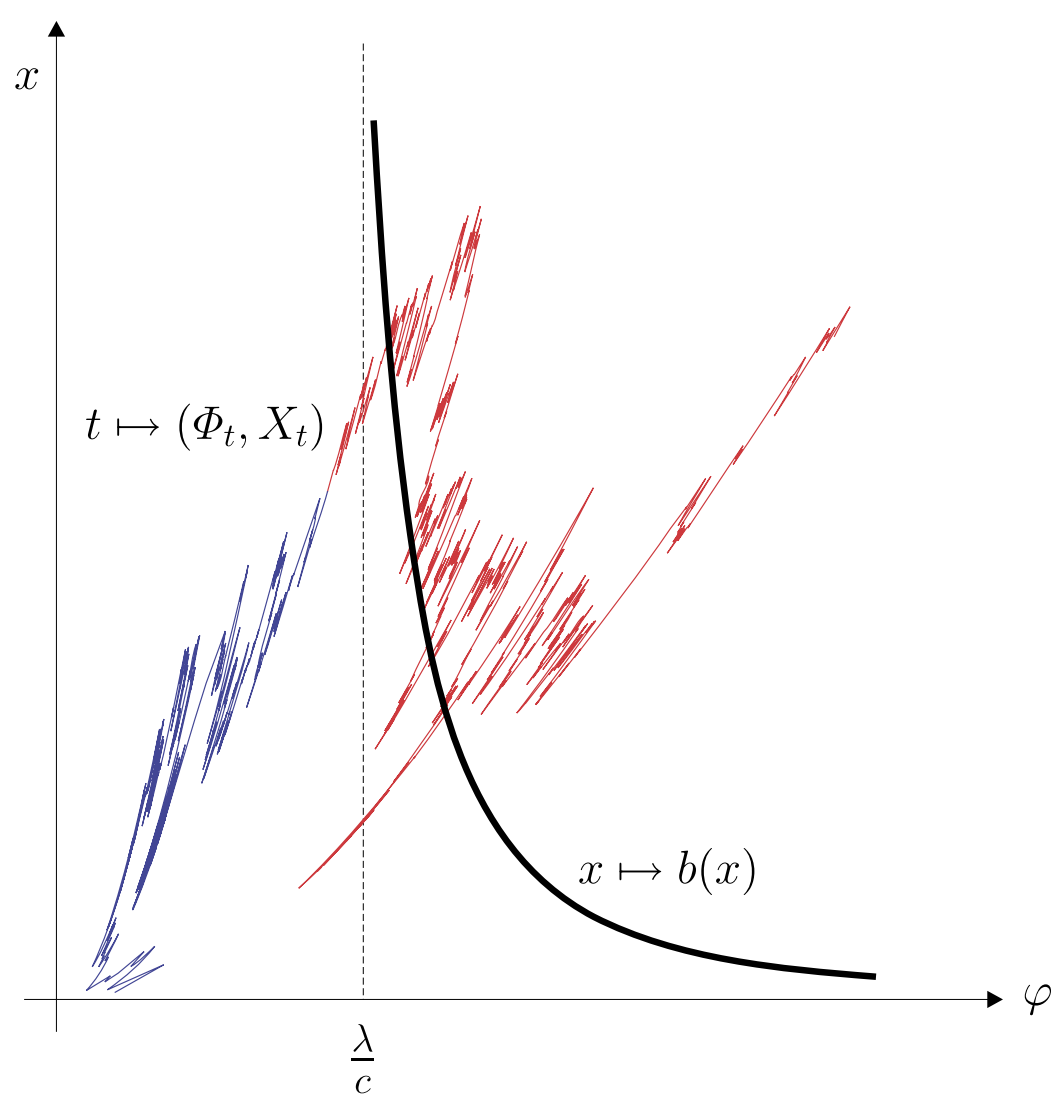

FIG. 3. Kinematics of the process $(\Phi, X)$ associated with the Bessel motion from Figure 2 above and location of the optimal stopping boundary $b$.

PROOF. 1. Existence. We first show that the optimal stopping boundary $b$ in the problem (4.19) solves the nonlinear integral equation (4.5). Recalling that $x \mapsto b(x)$ is a continuous and decreasing function satisfying $b(x) \geq \lambda / c$ for $x>0$ as established above, this will prove the existence of the solution to (4.5). For this, we will first show that Itô's formula is applicable to $\hat{V}$ composed with $(\Phi, X)$. Indeed, due to the bijective $C^{2}$ transformation (6.14) we see that it is enough to show that Itô's formula is applicable to $\tilde{V}$ composed with $(U, \Phi)$. For this, recall from the proof of Corollary 15 that $\tilde{V}$ is $C^{1,2}$ on the closure of $\tilde{C}$ and equals zero on $\tilde{D}$. We thus see that the local time-space formula from [13], Theorem 2.1 , is applicable to $\tilde{V}$ composed with $(U, \Phi)$ as long as $\tilde{b}(U)$ is shown to be a (continuous) semimartingale. For this, we know that $\tilde{b}$ is decreasing and $U$ is of bounded variation (both continuous), however, this is generally insufficient to conclude that $\tilde{b}(U)$ is of bounded variation itself (see [10]). We do know however that $U$ is monotone off the curve $z$ defined in (6.27) above. This fact can be used to establish the existence of a piecewise monotone process $U^{n}$ (i.e., monotone on each 
$\left[t_{i-1}, t_{i}\right)$ forming a partition of $[0, \infty)$ for $\left.i \geq 1\right)$ with continuous sample paths such that $U^{n}$ converges to $U$ uniformly on compacts of $[0, \infty)$, and the total variation of $U^{n}$ converges to the total variation of $U$ on each $[0, T]$ for $T>0$, both in probability as $n \rightarrow \infty$. One way of doing that is to take four continuous curves $z_{1}^{n}<z_{2}^{n}<z<z_{3}^{n}<z_{4}^{n}$ converging to $z$ uniformly on $[0, \infty)$ as $n \rightarrow \infty$, and if $(U, \Phi)$ starts outside the area between $z_{2}^{n}$ and $z_{3}^{n}$, consider the first hitting time of $(U, \Phi)$ to either $z_{2}^{n}$ or $z_{3}^{n}$, letting $U^{n}$ be equal to $U$ up to that time and freezing its value afterwards until $(U, \Phi)$ hits either $z_{1}^{n}$ or $z_{4}^{n}$, and then continuing by induction analogously; if $(U, \Phi)$ starts inside the area between $z_{2}^{n}$ and $z_{3}^{n}$ then $U^{n}$ can be set equal to the initial value of $U$ until $(U, \Phi)$ hits either $z_{1}^{n}$ or $z_{4}^{n}$, and we can then continue by induction as above. The resulting process is piecewise monotone and discontinuous, however, a simple straight-line approximation will make it continuous. Similar other constructions are also possible. Since each $\tilde{b}\left(U^{n}\right)$ is evidently of bounded variation and hence a (continuous) semimartingale, the local time-space formula from [13], Theorem 2.1, is applicable to $\tilde{V}$ composed with each $\left(U^{n}, \Phi\right)$, and due to the smooth-fit condition (11.29) we see that the local-time term in this formula vanishes, thus reducing it to Itô's formula for every $n \geq 1$ given and fixed. Letting then $n \rightarrow \infty$ and using the $C^{1,2}$ regularity of $\tilde{V}$ up to $\tilde{b}$ combined with the convergence relations of $U^{n}$ to $U$ (including the total variation in particular) this shows (see [5], Section 2.2) that Itô's formula extends from $\tilde{V}$ composed with each $\left(U^{n}, \Phi\right)$ for $n \geq 1$ to $\tilde{V}$ composed with $(U, \Phi)$ as needed. It follows therefore that Itô's formula is applicable to $\hat{V}$ composed with $(\Phi, X)$ as claimed.

Integrating further by parts this yields

$$
e^{-\lambda t} \hat{V}\left(\Phi_{t}, X_{t}\right)=\hat{V}(\varphi, x)+\int_{0}^{t} e^{-\lambda s}\left(\mathbb{L}_{\Phi, X} \hat{V}-\lambda \hat{V}\right)\left(\Phi_{s}, X_{s}\right) I\left(\Phi_{s} \neq X_{s}\right) d s
$$

$$
\begin{aligned}
& +\int_{0}^{t} e^{-\lambda s}\left[\gamma \frac{\Phi_{s}}{X_{s}} \hat{V}_{\varphi}\left(\Phi_{s}, X_{s}\right)+\hat{V}_{x}\left(\Phi_{s}, X_{s}\right)\right] d B_{s} \\
= & \hat{V}(\varphi, x)-\int_{0}^{t} e^{-\lambda s} H\left(\Phi_{s}, X_{s}\right) I\left(\Phi_{s}<b\left(X_{s}\right)\right) d s+M_{t}
\end{aligned}
$$

under $\mathrm{P}_{\varphi, x}^{\infty}$ with $(\varphi, x) \in[0, \infty) \times(0, \infty)$ given and fixed, where in the second equality we make use of (13.1) and (13.2), and $M_{t}=\int_{0}^{t} e^{-\lambda s}\left[\gamma\left(\Phi_{s} / X_{s}\right) \hat{V}_{\varphi}\left(\Phi_{s}\right.\right.$, $\left.\left.X_{S}\right)+\hat{V}_{x}\left(\Phi_{s}, X_{s}\right)\right] d B_{s}$ is a continuous local martingale for $t \geq 0$. Choosing a localisation sequence $\left(\tau_{n}\right)_{n \geq 1}$ of stopping times for $M$ and taking $\mathrm{E}_{\varphi, x}^{\infty}$ on both sides of (14.8) with $\tau_{n}$ in place of $t$ we find by the optional sampling theorem that

$$
\begin{aligned}
\mathrm{E}_{\varphi, x}^{\infty}[ & \left.e^{-\lambda \tau_{n}} \hat{V}\left(\Phi_{\tau_{n}}, X_{\tau_{n}}\right)\right] \\
& =\hat{V}(\varphi, x)-\mathrm{E}_{\varphi, x}^{\infty}\left[\int_{0}^{\tau_{n}} e^{-\lambda s} H\left(\Phi_{s}, X_{s}\right) I\left(\Phi_{s}<b\left(X_{s}\right)\right) d s\right]
\end{aligned}
$$

for $n \geq 1$. From (4.19), it is easily seen that

$$
-\frac{1}{c} \leq \hat{V} \leq 0
$$


on $[0, \infty) \times(0, \infty)$ so that the left-hand side in (14.9) tends to zero as $n \rightarrow \infty$. Moreover, from the form of $H$ given in (5.16) we see that the integral in (14.9) equals the difference of two integrals where the expected value of the second integral converges to a finite value. Letting $n \rightarrow \infty$ in (14.9) and using the monotone convergence theorem we therefore find that

$$
\hat{V}(\varphi, x)=\int_{0}^{\infty} e^{-\lambda s} \mathrm{E}_{\varphi, x}^{\infty}\left[H\left(\Phi_{s}, X_{s}\right) I\left(\Phi_{s}<b\left(X_{s}\right)\right)\right] d s
$$

for $(\varphi, x) \in[0, \infty) \times(0, \infty)$. Combining this expression with (14.4), we see that (14.6) holds as claimed. Setting $\varphi=b(x)$ in (4.15) for $x>0$ given and fixed, and using that $\hat{V}(b(x), x)=0$, we see that $b$ solves (14.5) and this completes the proof of existence.

2. Uniqueness. We next show that $b$ is a unique solution to (14.5) in the class of continuous and decreasing functions $x \mapsto b(x)$ satisfying $b(x) \geq \lambda / c$ for $x>0$. For this, take any continuous and decreasing function $c$ which solves (14.5) and satisfies $c(x) \geq \lambda / c$ for $x>0$. Motivated by (14.11) define the function $\hat{V}^{c}:[0, \infty) \times(0, \infty) \rightarrow \mathbb{R}$ by setting

$$
\hat{V}^{c}(\varphi, x)=\int_{0}^{\infty} e^{-\lambda s} \mathrm{E}_{\varphi, x}^{\infty}\left[H\left(\Phi_{s}, X_{s}\right) I\left(\Phi_{s}<c\left(X_{s}\right)\right)\right] d s
$$

for $(\varphi, x) \in[0, \infty) \times(0, \infty)$. Observe that $c$ solving (14.5) means exactly that $\hat{V}^{c}(c(x), x)=0$ for all $x>0$. Moreover, applying the strong Markov property of $(\Phi, X)$ at its first hitting time $\rho_{c}$ to $\{(c(x), x) \mid x>0\}$ under $\mathrm{P}_{\varphi, x}^{\infty}$ we see from the previous fact that $\hat{V}(\varphi, x)$ is finite as long as $\mathrm{E}_{\varphi, x}^{\infty}\left[\int_{0}^{\rho_{c}} H\left(\Phi_{s}, X_{s}\right) I\left(\Phi_{s}<\right.\right.$ $\left.\left.c\left(X_{s}\right)\right) d s\right]$ is finite. Letting $\rho$ denote the first hitting time of $(\Phi, X)$ to any given and fixed quadrant $\left\{\left(\varphi^{\prime}, x^{\prime}\right) \mid \varphi^{\prime} \geq \varphi_{1}\right.$ and $\left.x^{\prime} \geq x_{1}\right\}$ with $0 \leq \varphi<\varphi_{1}$ and $0<x<x_{1}$ such that its boundary lies above $c$ we see that the latter expectation is finite as long as $\mathrm{E}_{\varphi, x}^{\infty}\left[\int_{0}^{\rho} H\left(\Phi_{s}, X_{s}\right) I\left(\Phi_{s}<c\left(X_{s}\right)\right) d s\right]$ is finite. From (5.18), we see that the latter expectation is finite as long as $\lim _{n \rightarrow \infty} \mathrm{E}_{\varphi, x}^{\infty}\left[e^{-\lambda \rho_{n}} M\left(\Phi_{\rho_{n}}, X_{\rho_{n}}\right)\right]$ is finite (noting that the limit exists) where $\rho_{n}$ is any localised version of $\rho$ meeting the requirements of (5.18) with $\rho_{n} \uparrow \rho$ as $n \rightarrow \infty$. The fact that the latter limit is finite follows from (5.8) and (8.7) by the optional sampling theorem (upon localisation) combined with the facts that $\Phi_{\rho}$ and $X_{\rho}$ are constant on the set where $(\Phi, X)$ hits the quadrant at its vertical or horizontal side, respectively (recall from Section 8 that this happens with $\mathrm{P}_{\varphi, x}$-probability one). This shows that $\hat{V}^{c}$ is finite valued as claimed. On closer inspection, we also see that these arguments show that $\hat{V}^{c}$ is bounded on $[0, \infty) \times(0, \infty)$.

(i) We show that $\hat{V}^{c}(\varphi, x)=0$ for $(\varphi, x) \in[0, \infty) \times(0, \infty)$ such that $\varphi \geq$ $c(x)$. For this, take any such $(\varphi, x)$ and note that the Markov property of $(\Phi, X)$ implies that

$$
e^{-\lambda t} \hat{V}^{c}\left(\Phi_{t}, X_{t}\right)+\int_{0}^{t} e^{-\lambda s} H\left(\Phi_{s}, X_{s}\right) I\left(\Phi_{s}<c\left(X_{s}\right)\right) d s
$$


is a continuous martingale under $\mathrm{P}_{\varphi, x}^{\infty}$. Consider the stopping time

$$
\sigma_{c}=\inf \left\{t \geq 0 \mid \Phi_{t} \leq c\left(X_{t}\right)\right\}
$$

under $\mathrm{P}_{\varphi, x}^{\infty}$. Replacing $\sigma_{c}$ by $\sigma_{c} \wedge n$ in the sequel and letting $n \rightarrow \infty$ there is no restriction to assume that $\sigma_{c}$ is bounded. Since $\hat{V}^{c}(c(x), x)=0$ for all $x>0$, we see that $\hat{V}^{c}\left(\Phi_{\sigma_{c}}, X_{\sigma_{c}}\right)=0$ under $\mathrm{P}_{\varphi, x}^{\infty}$. Replacing $t$ by $\sigma_{c}$ in (14.13), taking $\mathrm{E}_{\varphi, x}^{\infty}$ on both sides and applying the optional sampling theorem, we thus find that

$$
\begin{aligned}
\hat{V}^{c}(\varphi, x)= & \mathrm{E}_{\varphi, x}^{\infty}\left[e^{-\lambda \sigma_{c}} \hat{V}^{c}\left(\Phi_{\sigma_{c}}, X_{\sigma_{c}}\right)\right] \\
& +\mathrm{E}_{\varphi, x}^{\infty}\left[\int_{0}^{\sigma_{c}} e^{-\lambda s} H\left(\Phi_{s}, X_{s}\right) I\left(\Phi_{s}<c\left(X_{s}\right)\right) d s\right]=0,
\end{aligned}
$$

where the second expectation equals zero by definition of $\sigma_{c}$. This shows that $\hat{V}^{c}$ equals zero above $c$ as claimed.

(ii) We show that $\hat{V}^{c}(\varphi, x) \geq \hat{V}(\varphi, x)$ for $(\varphi, x) \in[0, \infty) \times(0, \infty)$. For this, take any such $(\varphi, x)$ and consider the stopping time

$$
\tau_{c}=\inf \left\{t \geq 0 \mid \Phi_{t} \geq c\left(X_{t}\right)\right\}
$$

under $\mathrm{P}_{\varphi, x}^{\infty}$. Replacing $\tau_{c}$ by $\tau_{c} \wedge n$ in the sequel and letting $n \rightarrow \infty$ there is no restriction to assume that $\tau_{c}$ is bounded. We claim that $\hat{V}^{c}\left(\Phi_{\tau_{c}}, X_{\tau_{c}}\right)=0$ under $\mathrm{P}_{\varphi, x}^{\infty}$. Indeed, if $\varphi \geq c(x)$ then $\tau_{c}=0$ so that $\hat{V}^{c}\left(\Phi_{\tau_{c}}, X_{\tau_{c}}\right)=\hat{V}^{c}(\varphi, x)=0$ by (i) above. On the other hand, if $\varphi<c(x)$ then the claim follows since $\hat{V}^{c}(c(x)$, $x)=0$ as noted above. Replacing $t$ by $\tau_{c}$ in (14.13), taking $\mathrm{E}_{\varphi, x}^{\infty}$ on both sides and applying the optional sampling theorem, we thus find that

$$
\begin{aligned}
\hat{V}^{c}(\varphi, x)= & \mathrm{E}_{\varphi, x}^{\infty}\left[e^{-\lambda \tau_{c}} \hat{V}^{c}\left(\Phi_{\tau_{c}}, X_{\tau_{c}}\right)\right] \\
& +\mathrm{E}_{\varphi, x}^{\infty}\left[\int_{0}^{\tau_{c}} e^{-\lambda s} H\left(\Phi_{s}, X_{s}\right) I\left(\Phi_{s}<c\left(X_{s}\right)\right) d s\right] \\
= & \mathrm{E}_{\varphi, x}^{\infty}\left[\int_{0}^{\tau_{c}} e^{-\lambda s} H\left(\Phi_{s}, X_{s}\right) d s\right] \geq \hat{V}(\varphi, x),
\end{aligned}
$$

where the second equality follows by definition of $\tau_{c}$ and the inequality follows by definition of $\hat{V}$ in (4.19). This shows that $\hat{V}^{c} \geq \hat{V}$ on $[0, \infty) \times(0, \infty)$ as claimed.

(iii) We show that $c(x) \leq b(x)$ for all $x>0$. For this, suppose that there exists $x>0$ such that $c(x)>b(x)$. Fix any $\varphi \geq c(x)$ and consider the stopping time

$$
\sigma_{b}=\inf \left\{t \geq 0 \mid \Phi_{t} \leq b\left(X_{t}\right)\right\}
$$

under $\mathrm{P}_{\varphi, x}^{\infty}$. Replacing $\sigma_{b}$ by $\sigma_{b} \wedge n$ in the sequel and letting $n \rightarrow \infty$ there is no restriction to assume that $\sigma_{b}$ is bounded. Replacing $t$ by $\sigma_{b}$ in (14.13), taking $\mathrm{E}_{\varphi, x}^{\infty}$ on both sides and applying the optional sampling theorem, we find that

$$
\begin{aligned}
\hat{V}^{c}(\varphi, x)= & \mathrm{E}_{\varphi, x}^{\infty}\left[e^{-\lambda \sigma_{b}} \hat{V}^{c}\left(\Phi_{\sigma_{b}}, X_{\sigma_{b}}\right)\right] \\
& +\mathrm{E}_{\varphi, x}^{\infty}\left[\int_{0}^{\sigma_{b}} e^{-\lambda s} H\left(\Phi_{s}, X_{s}\right) I\left(\Phi_{s}<c\left(X_{s}\right)\right) d s\right] .
\end{aligned}
$$


Since $\varphi \geq c(x)$, we know that $\hat{V}^{c}(\varphi, x)=0$ by (i) above. Moreover, since $\hat{V}^{c} \geq \hat{V}$ by (ii) above and $\hat{V}\left(\Phi_{\sigma_{b}}, X_{\sigma_{b}}\right)=0$, we see that $\hat{V}^{c}\left(\Phi_{\sigma_{b}}, X_{\sigma_{b}}\right) \geq 0$. Combining the two conclusions in (14.19), we find that the following inequality holds:

$$
\mathrm{E}_{\varphi, x}^{\infty}\left[\int_{0}^{\sigma_{b}} e^{-\lambda s} H\left(\Phi_{s}, X_{s}\right) I\left(\Phi_{s}<c\left(X_{s}\right)\right) d s\right] \leq 0 .
$$

The fact that $c(x)>b(x)$ and the continuity of $b$ and $c$ imply that there exist $x_{1}<x$ and $x_{2}<x$ such that $c(y)>b(y)$ for all $y \in\left[x_{1}, x_{2}\right]$. Consequently, the $\mathrm{P}_{\varphi, x}^{\infty}$-probability of $(\Phi, X)$ spending a strictly positive amount of time below $c$ (with respect to Lebesgue measure) before hitting $b$ on $\left[x_{1}, x_{2}\right]$ is strictly positive. Combined with the fact that $b$ lies above $\lambda / c$ where $H$ is strictly positive, this forces the expectation in (14.20) to be strictly positive and provides a contradiction. Hence, $c \leq b$ on $(0, \infty)$ as claimed.

(iv) We show that $c(x)=b(x)$ for all $x>0$. For this, suppose that there exists $x>0$ such that $c(x)<b(x)$. Fix any $\varphi \in(c(x), b(x))$ and consider the stopping time

$$
\tau_{b}=\inf \left\{t \geq 0 \mid \Phi_{t} \geq b\left(X_{t}\right)\right\}
$$

under $\mathrm{P}_{\varphi, x}^{\infty}$. Replacing $\tau_{b}$ by $\tau_{b} \wedge n$ in the sequel and letting $n \rightarrow \infty$, there is no restriction to assume that $\tau_{b}$ is bounded. Replacing $t$ by $\tau_{c}$ in (14.8), taking $\mathrm{E}_{\varphi, x}^{\infty}$ on both sides and applying the optional sampling theorem, we find that

$$
\hat{V}(\varphi, x)=\mathrm{E}_{\varphi, x}^{\infty}\left[e^{-\lambda \tau_{b}} \hat{V}\left(\Phi_{\tau_{b}}, X_{\tau_{b}}\right)\right]+\mathrm{E}_{\varphi, x}^{\infty}\left[\int_{0}^{\tau_{b}} e^{-\lambda s} H\left(\Phi_{s}, X_{s}\right) d s\right] .
$$

Similarly, replacing $t$ by $\tau_{b}$ in (14.13), taking $\mathrm{E}_{\varphi, x}^{\infty}$ on both sides and applying the optional sampling theorem, we find that

$$
\begin{aligned}
\hat{V}^{c}(\varphi, x)= & \mathrm{E}_{\varphi, x}^{\infty}\left[e^{-\lambda \tau_{b}} \hat{V}^{c}\left(\Phi_{\tau_{b}}, X_{\tau_{b}}\right)\right] \\
& +\mathrm{E}_{\varphi, x}^{\infty}\left[\int_{0}^{\tau_{b}} e^{-\lambda s} H\left(\Phi_{s}, X_{s}\right) I\left(\Phi_{s}<c\left(X_{S}\right)\right) d s\right] .
\end{aligned}
$$

Since $\hat{V}(\varphi, x)<0$ due to $\varphi<b(x)$ and $\hat{V}\left(\Phi_{\tau_{b}}, X_{\tau_{b}}\right)=0$, we see that the final expectation in (14.22) is strictly negative. Since $\hat{V}^{c}(\varphi, x)=0$ by (i) above due to $\varphi>c(x)$, and $\hat{V}^{c}\left(\Phi_{\tau_{b}}, X_{\tau_{b}}\right)=0$ again by (i) above due to $b \geq c$ by (iii) above, we see that the final expectation in (14.23) equals zero. Combining the two conclusions on the final expectations in (14.22) and (14.23), it follows that

$$
\mathrm{E}_{\varphi, x}^{\infty}\left[\int_{0}^{\tau_{b}} e^{-\lambda s} H\left(\Phi_{s}, X_{s}\right) I\left(\Phi_{s} \geq c\left(X_{s}\right)\right) d s\right]<0 .
$$

But then as in (iii) above the continuity of $b$ and $c$ combined with the fact that $c$ lies above $\lambda / c$ where $H$ is strictly positive forces the expectation in (14.24) to be strictly positive and provides a contradiction. Thus, $c=b$ on $(0, \infty)$ as claimed and the proof is complete. 
Collecting the results derived throughout we now disclose the solution to the initial problem.

COROllary 20. With initial point $x>0$ of the process $X$ solving (3.1) + (3.2) given and fixed, the value function of the initial problem (3.6) is given by

$$
V(\pi)=(1-\pi)\left[1+c \hat{V}\left(\frac{\pi}{1-\pi}, x\right)\right]
$$

for $\pi \in[0,1]$ where the function $\hat{V}$ is given by (14.6) above. The optimal stopping time in the initial problem (3.6) is given by

$$
\begin{aligned}
\tau_{*}= & \inf \left\{t \geq 0 \mid\left(\frac{X_{t}}{x}\right)^{\gamma} e^{\lambda t-\beta \int_{0}^{t} \frac{d s}{X_{s}^{2}}}\left(\frac{\pi}{1-\pi}\right.\right. \\
& \left.\left.+\lambda \int_{0}^{t} \frac{d s}{\left(\frac{X_{s}}{x}\right)^{\gamma} e^{\lambda s-\beta \int_{0}^{s} \frac{d r}{X_{r}^{2}}}}\right) \geq b\left(X_{t}\right)\right\},
\end{aligned}
$$

where $\gamma=\left(\delta_{1}-\delta_{0}\right) / 2, \beta=\left(\delta_{1}-\delta_{0}\right)\left(\delta_{1}+\delta_{0}-4\right) / 8$ and $b$ is a unique solution to the integral equation (14.5) in the class of continuous and decreasing functions $x \mapsto b(x)$ satisfying $b(x) \geq \lambda / c$ for $x>0$.

PROOF. The identity (14.25) follows by combining (4.11) + (4.12) in Proposition 2 with the result of Theorem 19. The explicit form (14.26) follows from (14.7) in Theorem 19 combined with (3.12) and (6.19) + (6.20) completing the proof.

\section{REFERENCES}

[1] Assing, S., JACKA, S. and OCEJo, A. (2014). Monotonicity of the value function for a twodimensional optimal stopping problem. Ann. Appl. Probab. 24 1554-1584. MR3211004

[2] Bayraktar, E., DAyanik, S. and Karatzas, I. (2005). The standard Poisson disorder problem revisited. Stochastic Process. Appl. 115 1437-1450. MR2158013

[3] De Angelis, T. and PeskiR, G. (2015). Global $C^{1}$ regularity of the value function in optimal stopping problems. Research Report No. 6, Probab. Statist. Group Manchester. To appear.

[4] DU Toit, J. and Peskir, G. (2009). Selling a stock at the ultimate maximum. Ann. Appl. Probab. 19 983-1014. MR2537196

[5] Engelbert, H.-J. and Peskir, G. (2014). Stochastic differential equations for sticky Brownian motion. Stochastics 86 993-1021. MR3271518

[6] Feller, W. (1952). The parabolic differential equations and the associated semi-groups of transformations. Ann. of Math. (2) 55 468-519. MR0047886

[7] Ferreyra, G. and Sundar, P. (2000). Comparison of solutions of stochastic equations and applications. Stoch. Anal. Appl. 18 211-229. MR1747185

[8] GapeeV, P. V. and PeskiR, G. (2006). The Wiener disorder problem with finite horizon. Stochastic Process. Appl. 116 1770-1791. MR2307058

[9] GapeEV, P. V. and ShIRYAEV, A. N. (2013). Bayesian quickest detection problems for some diffusion processes. Adv. in Appl. Probab. 45 164-185. MR3077545 
[10] Josephy, M. (1981). Composing functions of bounded variation. Proc. Amer. Math. Soc. 83 354-356. MR0624930

[11] Lieberman, G. M. (1996). Second Order Parabolic Differential Equations. World Scientific, River Edge, NJ. MR1465184

[12] PeskiR, G. (2005). On the American option problem. Math. Finance 15 169-181. MR2116800

[13] PeskiR, G. (2007). A change-of-variable formula with local time on surfaces. In Séminaire de Probabilités XL. Lecture Notes in Math. 1899 69-96. Springer, Berlin. MR2408999

[14] PeskiR, G. (2015). Continuity of the optimal stopping boundary for two-dimensional diffusions. Probab. Statist. Group Manchester. Research Report No. 4. To appear.

[15] Peskir, G. and Shiryaev, A. (2006). Optimal Stopping and Free-Boundary Problems. Birkhäuser, Basel. MR2256030

[16] RevuZ, D. and Yor, M. (1999). Continuous Martingales and Brownian Motion, 3rd ed. Grundlehren der Mathematischen Wissenschaften [Fundamental Principles of Mathematical Sciences] 293. Springer, Berlin. MR1725357

[17] Rogers, L. C. G. and Williams, D. (2000). Diffusions, Markov Processes, and Martingales: Itô Calculus. Vol. 2. Cambridge Univ. Press, Cambridge. MR1780932

[18] Shiryaev, A. N. (1961). The problem of the most rapid detection of a disturbance in a stationary process. Sov. Math., Dokl. 2 795-799.

[19] Shiryaev, A. N. (2010). Quickest detection problems: Fifty years later. Sequential Anal. 29 345-385. MR2747531

[20] ShiryayeV, A. N. (1978). Optimal Stopping Rules. Springer, New York. MR0468067

[21] VOLKONSKIĬ, V. A. (1958). Random substitution of time in strong Markov processes. Theory Probab. Appl. 3 310-326. MR0100919

SCHOOL OF MATHEMATICS

THE UNIVERSITY OF MANCHESTER

OXFORD ROAD

MANCHESTER M13 9PL

UNITED KINGDOM

E-MAIL: peter.johnson-3@manchester.ac.uk goran@maths.man.ac.uk 